\title{
Paleoenvironmental reconstruction of the Upper Mississippian Reynolds Limestone in the Central Appalachian Basin of West Virginia
}

\author{
Simon L. Cole III \\ West Virginia University
}

Follow this and additional works at: https://researchrepository.wvu.edu/etd

\section{Recommended Citation}

Cole, Simon L. III, "Paleoenvironmental reconstruction of the Upper Mississippian Reynolds Limestone in the Central Appalachian Basin of West Virginia" (2005). Graduate Theses, Dissertations, and Problem Reports. 2168.

https://researchrepository.wvu.edu/etd/2168

This Thesis is protected by copyright and/or related rights. It has been brought to you by the The Research Repository @ WVU with permission from the rights-holder(s). You are free to use this Thesis in any way that is permitted by the copyright and related rights legislation that applies to your use. For other uses you must obtain permission from the rights-holder(s) directly, unless additional rights are indicated by a Creative Commons license in the record and/ or on the work itself. This Thesis has been accepted for inclusion in WVU Graduate Theses, Dissertations, and Problem Reports collection by an authorized administrator of The Research Repository @ WVU. For more information, please contact researchrepository@mail.wvu.edu. 
Paleoenvironmental Reconstruction of the Upper Mississippian Reynolds Limestone in the Central Appalachian Basin of West Virginia.

\author{
Simon L. Cole III \\ Thesis Submitted to the Eberly College of Arts and Sciences \\ at West Virginia University \\ in partial fulfillment of the requirements \\ for the Degree of
}

Master of Science

in

Geology

Thomas Kammer, Ph.D., Chair

Richard Smosna, Ph.D.

Ronald McDowell, Ph.D.

Department of Geology and Geography

Morgantown, WV

2005 


\section{ABSTRACT \\ Paleoenvironmental Reconstruction of the Upper Mississippian Reynolds Limestone in the Central Appalachian Basin of West Virginia}

\section{Simon L. Cole III}

The Upper Mississippian strata in West Virginia, southern Pennsylvania, and western Maryland are predominantly composed of interbedded limestones and shales. The focus of this study, the Reynolds Limestone, is a formal member of the lower part of the Bluefield Formation, in the Mauch Chunk Group, of the Chesterian Series.

The rock types composing the Reynolds Limestone include light to medium grey, argillaceous wackestones to skeletal packstones and red to grey shales. This unit is sporadically exposed throughout the study area. Unit thickness varies across the study area from less than 10 $\mathrm{m}$ in the north to over $100 \mathrm{~m}$ in the south.

Seven localities were investigated in the present study. The area of investigation includes localities in Garrett County, Maryland; Fayette County, Pennsylvania; and Grant, Monongalia, Pocahontas, and Randolph counties, West Virginia.

This research addresses facies changes in terms of sea level fluctuations and basinal settings during the deposition of the Reynolds Limestone. Specifically, this research consists of the measurement and description of the seven field localities and thin section analysis of carbonate units within the identified study interval.

Primary objectives were to determine the depositional history of the Reynolds Limestone based on the paleoecology and stratigraphy of the strata. These objectives were achieved through detailed outcrop measurement and thin section analysis of carbonate units.

Two composite models were created to illustrate the relationships between the facies identified in this study. They illustrate how the facies are laterally related and the complexities between the various depositional environments. 
Results indicate the Reynolds Limestone was deposited during several, small-scale transgressive/regressive events. The Reynolds is regionally correlative to the Glen Dean Limestone of the Illinois Basin. This correlation suggests that large-scale glacial effects were controlling eustatic sea level fluctuations in the Late Mississippian. An early onset glacial event is recorded by the Lillydale Shale and an interglacial period by the Reynolds Limestone. The data show structural controls played an important role in the deposition of the strata in the Reynolds interval but not on sea level fluctuations. In southern West Virginia, there is a significant thickening of strata resulting from filling of a rapidly subsiding basin. It has been determined based on the paleoshoreline orientation and its relation to sea level fluctuations, that the Reynolds Limestone can be considered a time correlative unit. 


\section{TABLE OF CONTENTS}

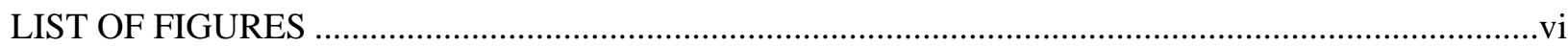

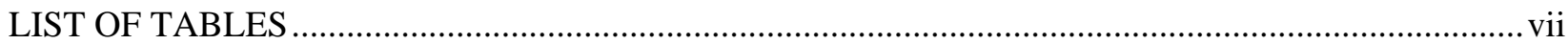

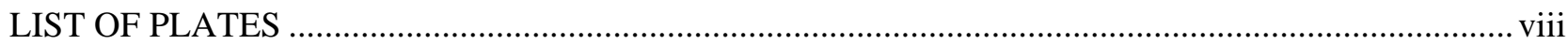

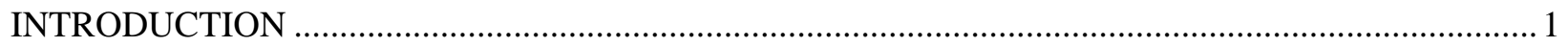

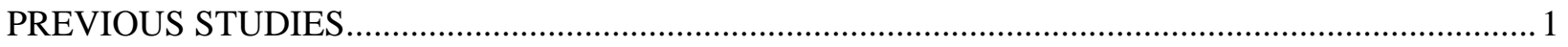

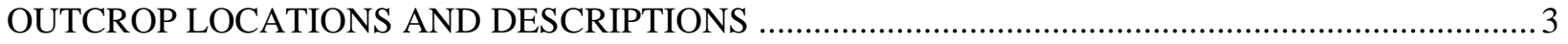

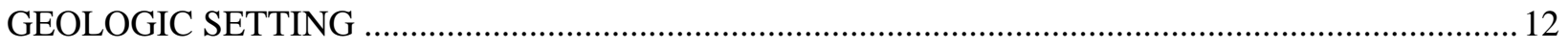

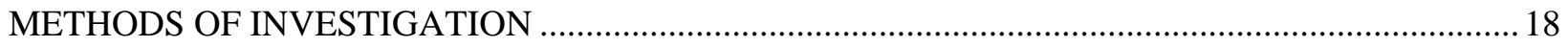

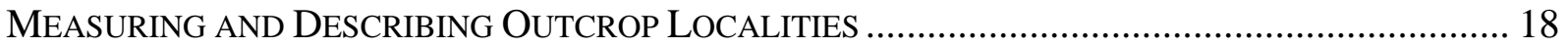

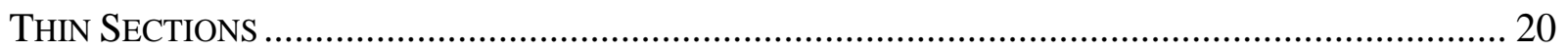

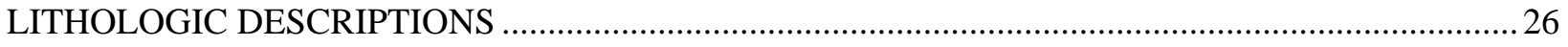

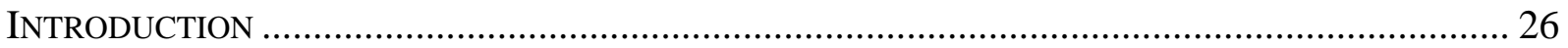

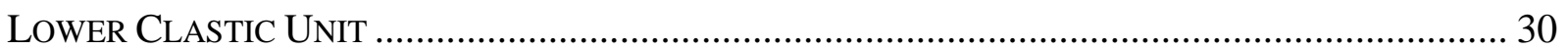

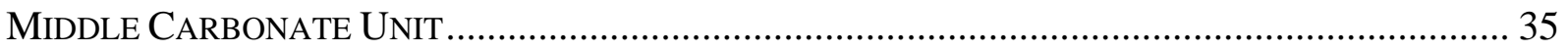

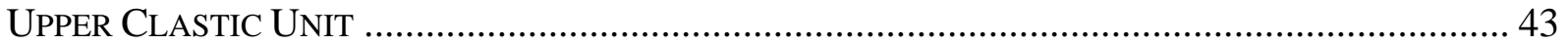

LITHOFACIES ASSOCIATIONS AND INTERPRETATIONS OF DEPOSITIONAL

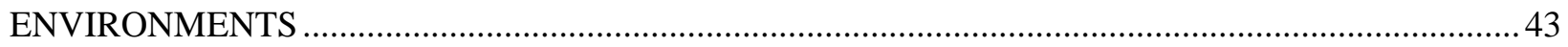

MiXed CARBONATE AND SILICICLASTIC Association .......................................................... 43

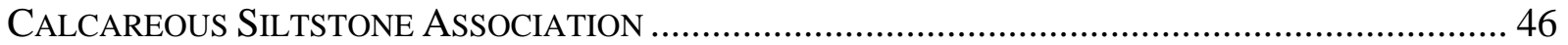

Peloidal Mudstone / Packstone Association .................................................................. 50

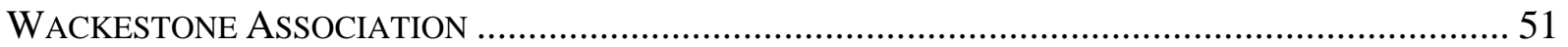

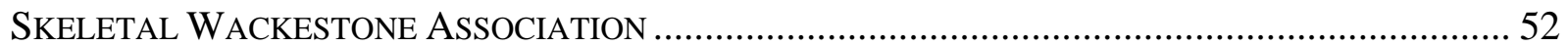




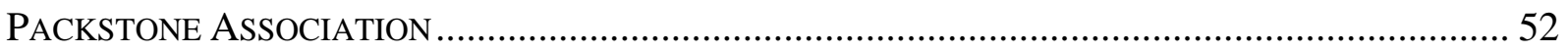

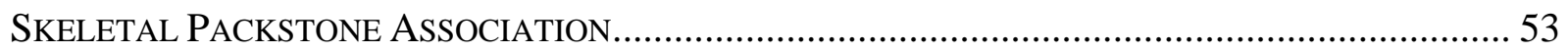

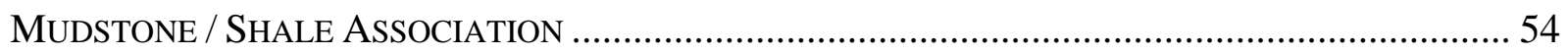

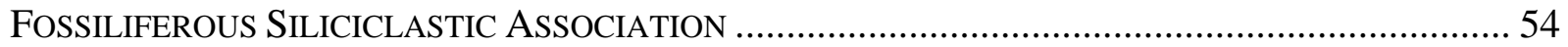

NON-FOSSILIFEROUS SILICICLASTIC ASSOCIATION ........................................................ 55

DEPOSITIONAL MODEL FOR ENVIRONMENTAL INTERPRETATIONS ..................................... 55

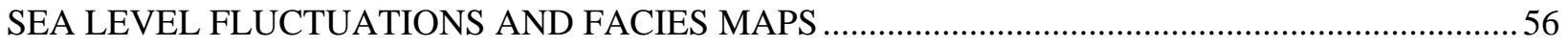

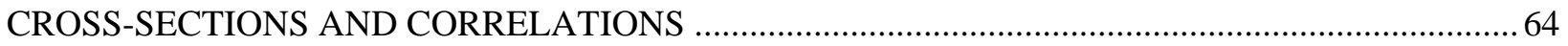

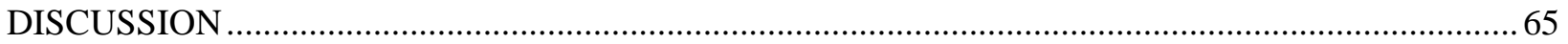

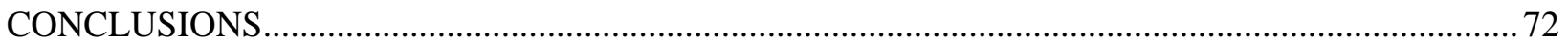

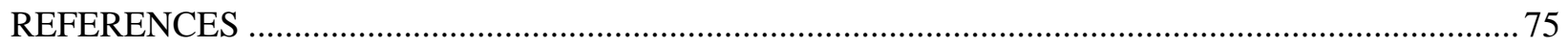

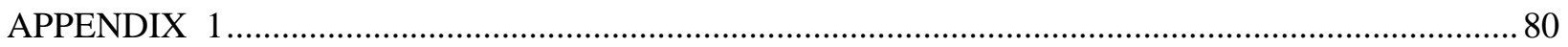

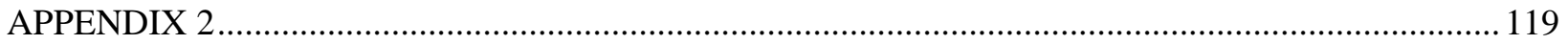

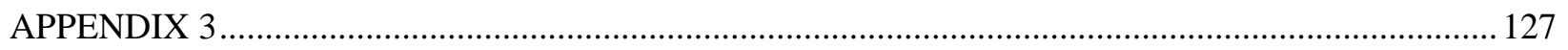

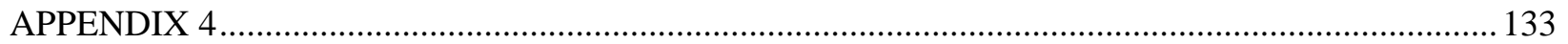




\section{LIST OF FIGURES}

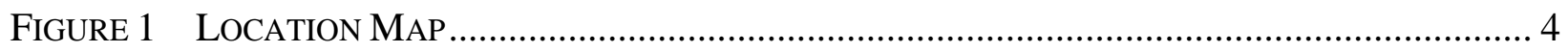

Figure 2 A Generalized Paleogeographic Map .......................................................... 13

Figure 3 Paleoshoreline Positions During Mauch Chunk Deposition ......................... 14

Figure 4 Basinal Conditions During Mauch Chunk Deposition .................................. 15

FigURE 5 StRATIGRAPHIC Position OF THE REYNOLDS LiMESTONE ..................................... 16

Figure 6 Stratigraphic NOMENClAtURE OF THE UPPER MisSISSIPPIAN STRATA .................. 17

FIGURE 7 MODELS FOR ENVIRONMENTAL INTERPRETATION ............................................... 47

FIGURE 8 ENVIRONMENTAL SETTING MAP ............................................................. 57

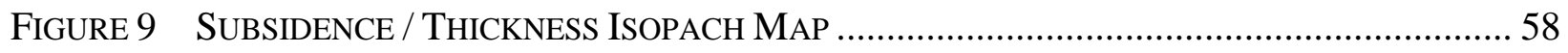

Figure 10 Facies MaP FOR THE Top OF THE LiLlydale Shale ........................................... 60

Figure 11 FACIES MAP FOR THE BASE Of THE REYNOLDS LiMESTONE ................................... 61

Figure 12 Facies Map AT The MaXimum Point Of SEA LEVEL ........................................... 62

Figure 13 Facies Map of Regression After Maximum SeA LeVel .................................. 63

FigURE 14 GREENBRIER LiMESTONE ISOPACH THICKNESS MAP .......................................... 68

Figure 15 Composite SEA LEVEl CURVES For EARLY AND MIDDLE CHESTERIAN ....................69

FIGURE 16 SEPARATION OF THE REYNOLDS AND GLENRAY LIMESTONES................................ 71

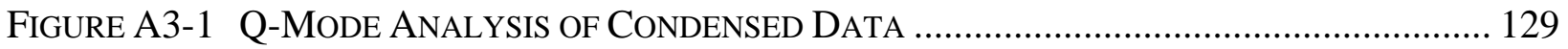

Figure A3-2 Q-Mode AnAlysis OF Syndepositional Data …........................................ 131 


\section{LIST OF TABLES}

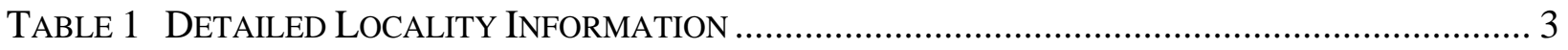

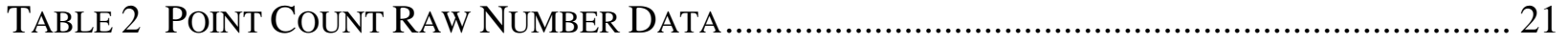

TABle 3 Point Count Percent Data ............................................................................... 22

TABLE 4 OBSERVATIONAL DATA FOR ThIN SECTION UNITS .............................................. 23-25

TABle 5 Point Count Percent CATEgory Totals …........................................................... 27

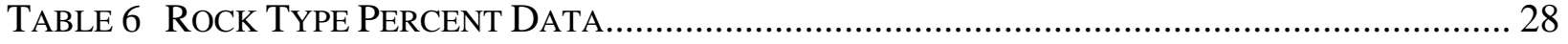

Table 7 Average Point Count Data Based on Rock Type .............................................. 29

TABle 8 EnVIRONMENTAL InTERPRETAtions of Thin SECTION Units By FACIES............... 48-49 


\section{LIST OF PLATES}

Plate 1 LAKE LynN, CharcoAl HILl, AND SCHERR SECTIONS ............................................ 5

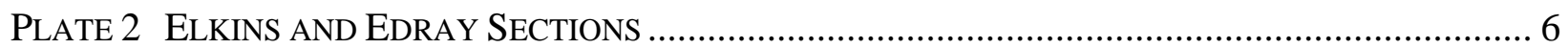

PLATE 3 EdRAy SECTION (LiLLydale SHALE) ............................................................... 7

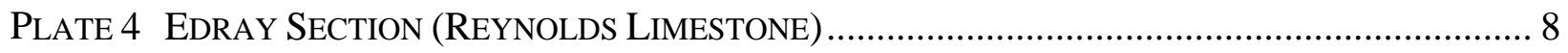

Plate 5 Edray Section (Unit 6 Tidal Channels) and Droop Mountain SEction

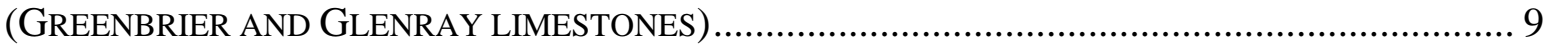

Plate 6 Droop Mountain Section (Glenray Limestone, Webster Springs SAndstone,

AND THE BASE OF THE REYNOLDS LIMESTONE) .................................................. 10

Plate 7 Droop Mountain Section (Reynolds Limestone) ….......................................... 11

Plate 8 Thin Sections - Calcareous Siltstone And Microbial Boundstone ................ 34

Plate 9 Thin Sections - Wackestone, Skeletal Wackestone, and Skeletal Packstone 36

Plate 10 Thin Sections - Skeletal Packstone, Peloidal Packstone, and Poorly

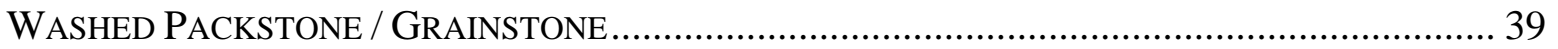

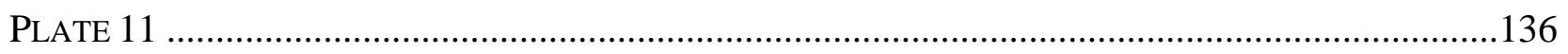




\section{INTRODUCTION}

This research addresses changes in depositional facies and interpreted sea level fluctuations in the stratigraphic interval from the top of the Mississippian Greenbrier Limestone into the strata overlying the Reynolds Limestone Member of the Mauch Chunk Formation. In particular, this consists of: a) the identification and documentation of fossils occurring within this stratigraphic interval; b) the measurement and description of all stratigraphic units; and c) the interpretation and analysis of facies relationships across the study area. The goal of this research was to gain an understanding of facies relationships in the context of interpreted sea level fluctuations in order to better understand the depositional history of the Reynolds Limestone.

Principal objectives were to document lithologic, stratigraphic, and faunal variation across the study area in order to identify sea level fluctuations and facies changes. The collection, documentation, and analysis of stratigraphic and taxonomic data from both field observations and laboratory work together with paleoecologic literature and principles, provided insight into the facies relationships across the study area.

\section{PREVIOUS STUDIES}

Initial reports of the Reynolds Limestone (Little Lime) come from geologic survey county reports and refer to this unit only in reference to the Greenbrier Limestone (Big Lime). These county reports were referenced and used to locate outcrops and find exposures across the study area: Fayette Co., PA (Hickok and Moyer, 1971); Grant Co., WV (Reger et al, 1924); Pocahontas Co., (Price, 1929); Randolph Co., WV (Reger et al, 1918, 1920). 
Manspeizer (1958) was among the first to describe the paleontology and stratigraphy of the Bluefield Formation later followed by Humphreville (1981). Busanus (1974) studied the paleontology and paleoecology of the Mauch Chunk Group. Presley (1977) studied depositional settings in the Upper Mississippian. He showed that the Mauch Chunk group was deposited in fluvial and fluvial-deltaic environments. Arkle et al. (1979) followed up by looking at the entire Mississippian System.

Brezenski (1984) investigated the lithostratigraphy and paleoecology of Upper Mississippian strata and their relation to sea level fluctuations. Tissue (1986) conducted a paleoecologic analysis of the Greenbrier and lower Mauch Chunk rocks and the environmental parameters controlling them. His study showed the Mauch Chunk clastics swamped the carbonate system and resulted in a gradual southwestward regression of the Greenbrier sea. Corbitt (1986) looked specifically at the Reynolds Limestone and conducted a petrographic analysis to identify various depositional environments within the unit. Her findings show the Reynolds was deposited in a series of shoaling upward sequences on a broad shallow carbonate shelf dominated by terrigenous clastic sediments. Carney (1987) followed with a petrographic study primarily focusing on the Greenbrier Limestone but also included the Reynolds. Her study documented the depositional setting and burial history surrounding the Greenbrier Limestone. Christopher (1992) conducted a regional study of the paleoecology of the Bluefield Formation in southern West Virginia and was the first to use statistical analyses to support his findings. Lake (1998) conducted the most recent investigation into the Reynolds Limestone strata looking specifically at the Wymps Gap to Reynolds Limestone interval. His results showed that his taxonomic gradients were strongly influenced by the physical environment as opposed to being 
controlled by biologic interactions. This analysis was based on the faunal composition of the units and like Christopher (1992) used statistical analyses to support his findings.

\section{OUTCROP LOCATIONS AND DESCRIPTIONS}

The study area (Fig. 1) includes seven outcrops extending from southwestern Pennsylvania to southern West Virginia. The localities are: (1) Hopwood - Fayette Co., PA; (2) Lake Lynn - Fayette Co., PA (Plate 1a); (3) Charcoal Hill - Garrett Co., MD (Plate 1b); (4) Scherr - Grant Co., WV (Plate 1c); (5) Elkins - Randolph Co., WV (Plate 2a); (6) Edray Pocahontas Co., WV (Plate 2b, 3a-c, 4a-c. 5a); (7) Droop Mountain - Pocahontas Co., WV (Plates 5b-c, 6a-c, 7a). More detailed location information is provided in Table 1 (Fig. 1). These outcrops were selected because of their complete exposure of Reynolds Limestone from the top of the Greenbrier Limestone into the overlying Mauch Chunk clastics and their geographic distribution across the study area.

TABLE 1

\begin{tabular}{|c|c|c|c|c|c|}
\hline \multirow[t]{2}{*}{ Outcrop } & \multirow[t]{2}{*}{ Location } & \multicolumn{2}{|c|}{ GPS Coordinates } & \multirow[t]{2}{*}{ Quadrangle } & \multirow[t]{2}{*}{ Notes } \\
\hline & & Easting & Northing & & \\
\hline Hopwood & $\begin{array}{l}\text { Hopwood, PA US Rt. } 40 \text { below } \\
\text { Mt. Summit. }\end{array}$ & 612641 & 4413701 & Uniontown, PA & $\begin{array}{l}\text { Truck ramp near Lick Hollow Picnic } \\
\text { Area entrance. }\end{array}$ \\
\hline Lake Lynn & $\begin{array}{l}\text { Lake Lynn Laboratory, US } \\
\text { Bureau of Mines, Gans, PA }\end{array}$ & 605570 & 4397500 & Lake Lynn, PA-WV & In highwall above portal \#4 entrance. \\
\hline $\begin{array}{l}\text { Charcoal } \\
\text { Hill }\end{array}$ & Oakland, MD & 632479 & 4358878 & Table Rock, MD & $\begin{array}{l}\text { Southwest of town near the } \\
\text { Youghigheny River. }\end{array}$ \\
\hline Scherr & Rt. 42 near Scherr, WV & 655018 & 4341345 & Greenland Gap, WV & $\begin{array}{l}\text { Section located above an old } \\
\text { Greenbrier quarry. }\end{array}$ \\
\hline Elkins & $\begin{array}{l}\text { JF Allen Quarry, Randolph Co., } \\
\text { WV }\end{array}$ & 594221 & 4310155 & Elkins, WV & $\begin{array}{l}\text { Active quarry located just west of } \\
\text { Elkins, WV and south of US } 33 \text {. }\end{array}$ \\
\hline Edray & US Rt. 219 Pocahontas Co., WV & 578794 & 4239102 & Edray, WV & $\begin{array}{l}\text { Begin at bend in road north of scenic } \\
\text { overlook. }\end{array}$ \\
\hline Droop Mtn. & US Rt. 219 Pocahontas Co., WV & 563826 & 4218164 & Droop, WV & $\begin{array}{l}\text { Begin at north entrance to Droop Mtn. } \\
\text { State Park. }\end{array}$ \\
\hline
\end{tabular}

Location information for this table was compiled from pre-existing thesis data and actual field work (Corbitt, 1986; Tissue, 1986; Carney, 1987; Christopher, 1992; Lake, 1998). 


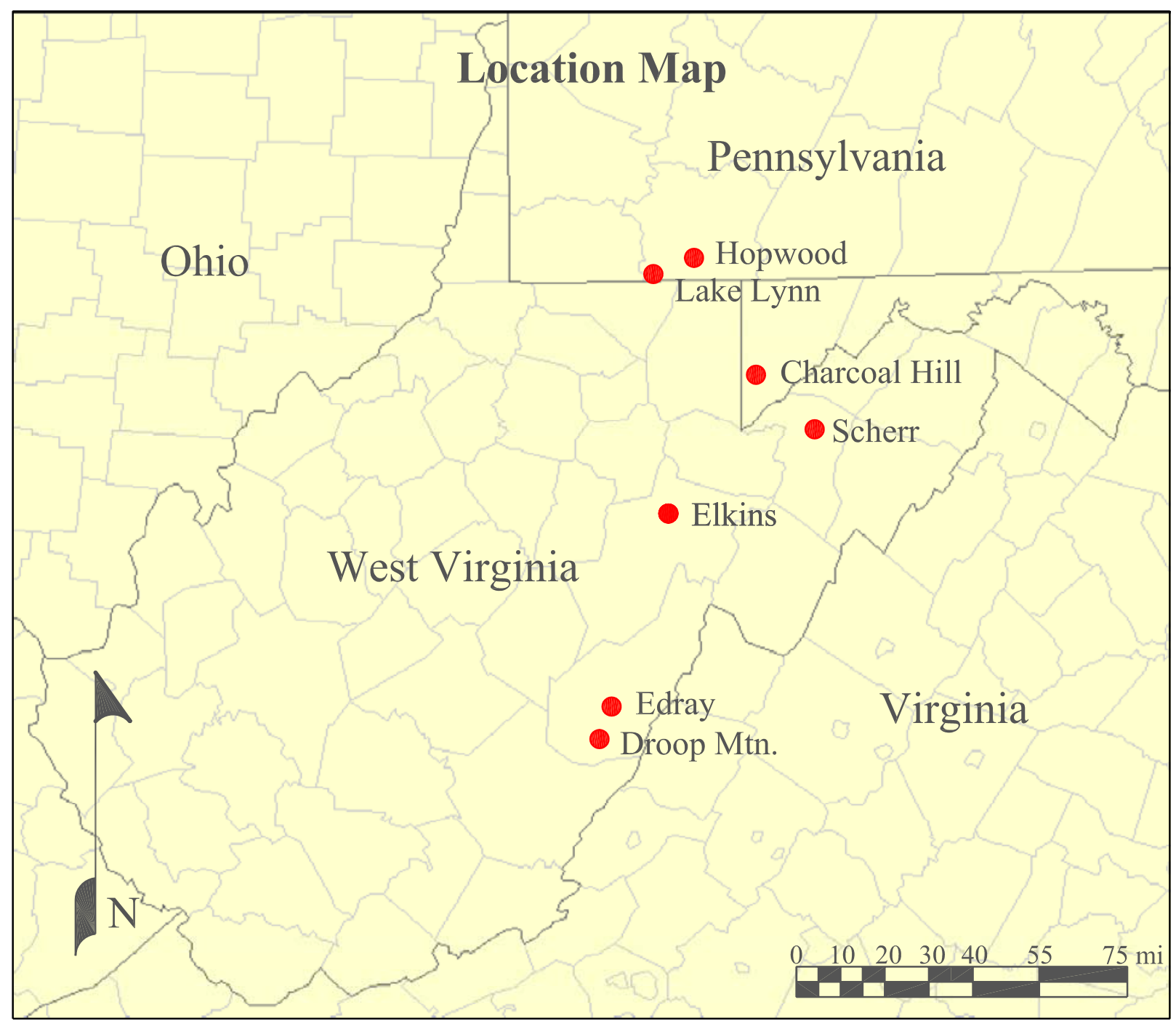

Figure 1. Locations of the outcrops used in this study. More detailed locality information is provided in Table 1. 


\section{Plate 1}

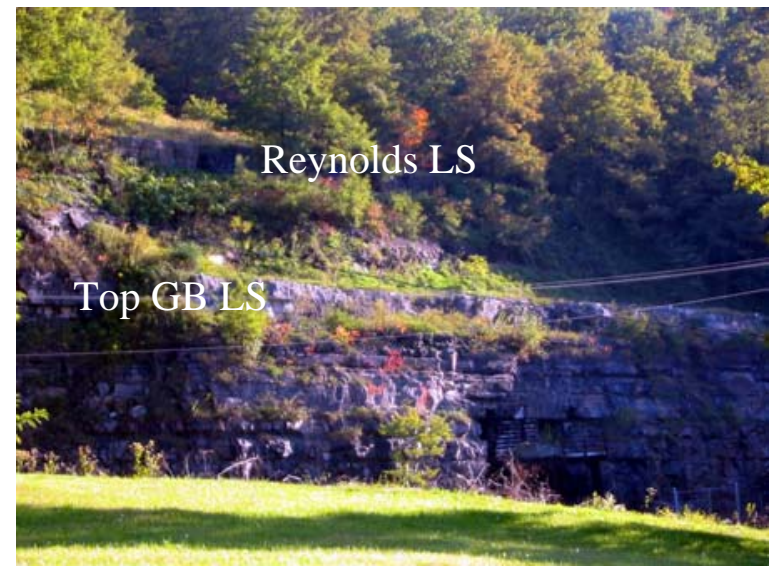

1a. Lake Lynn Laboratory section.

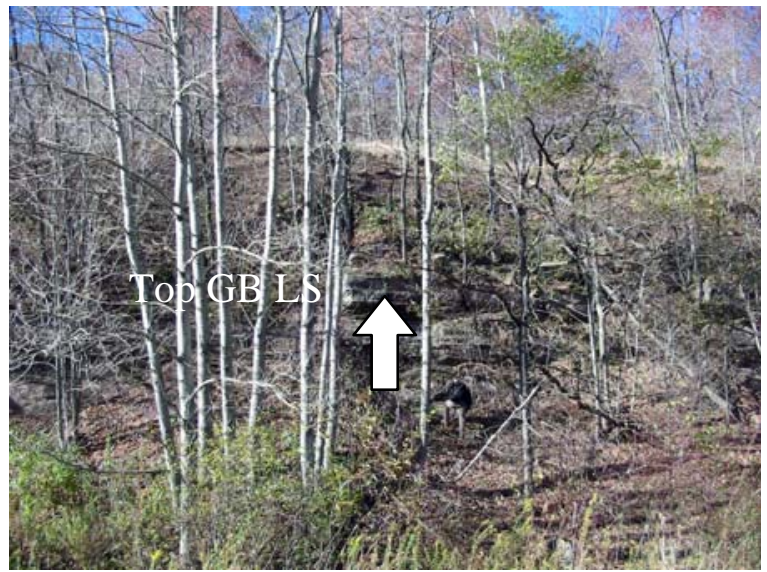

1b. Charcoal Hill section.

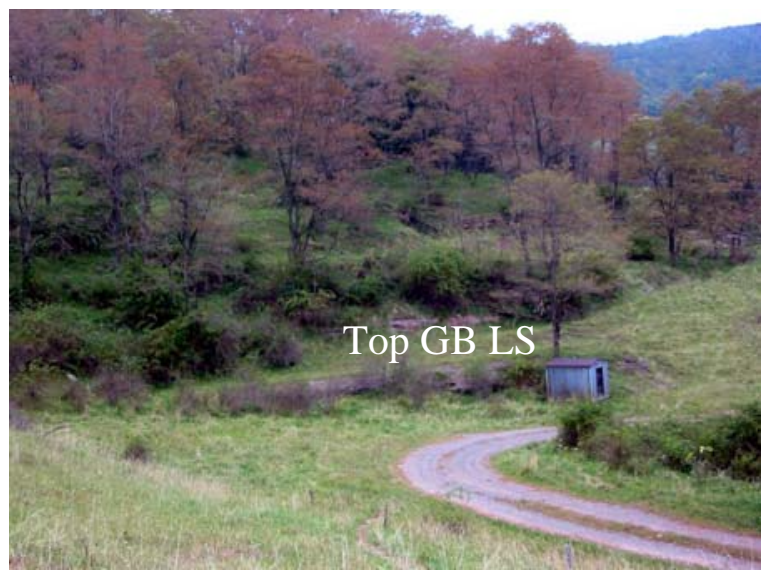

1c. Scherr section. 


\section{Plate 2}

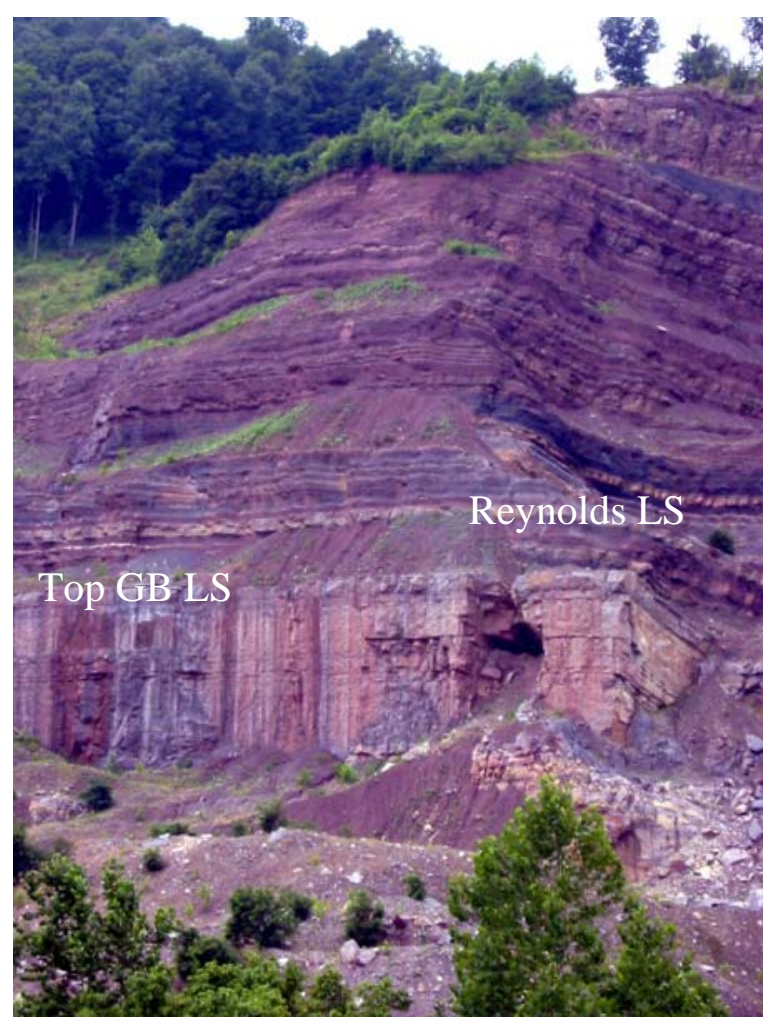

2a. Elkins Section at J.F. Allen Quarry.

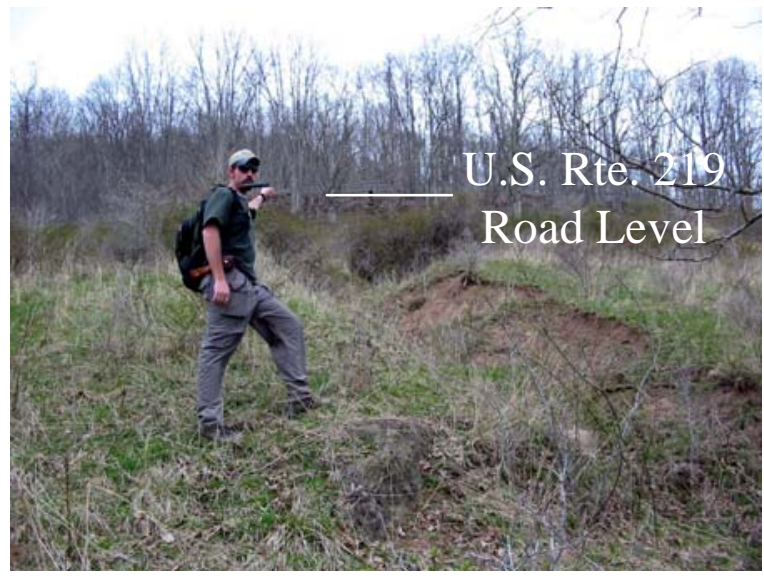

2b. Edray - Top of the Greenbrier Limestone. 


\section{Plate 3}

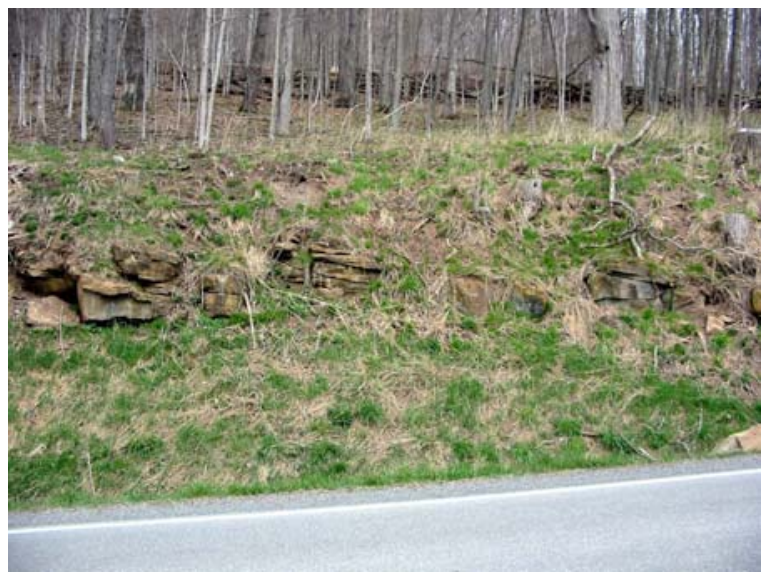

3a. Edray - Marine sandstone in the Lillydale Shale.

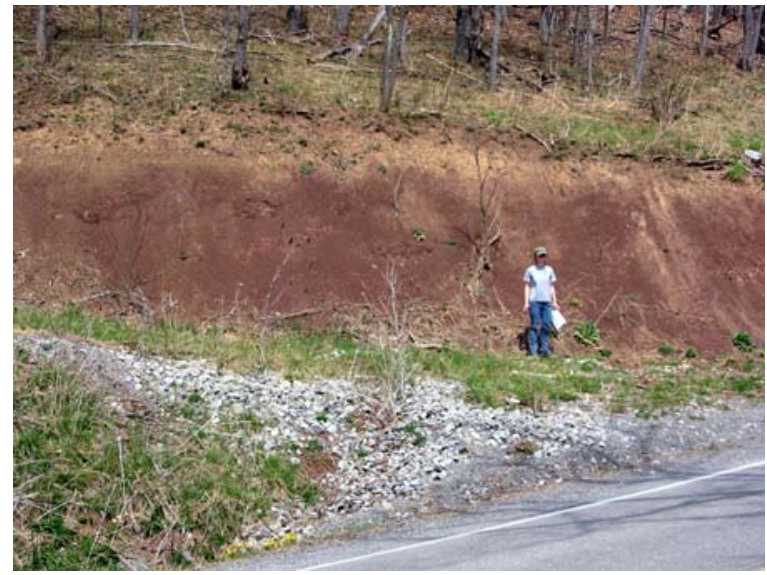

3b. Edray - Red beds near the top of the Lillydale Shale interval.

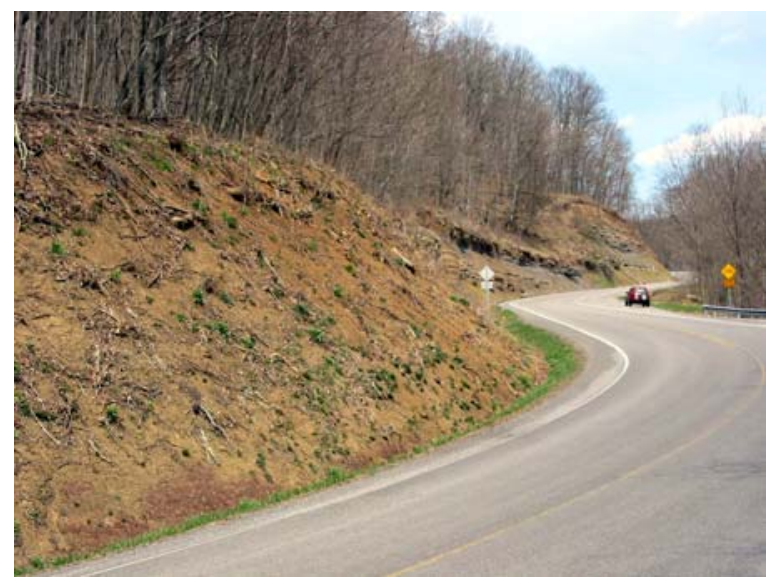

3c. Edray - Top of Lillydale Shale interval. 


\section{Plate 4}

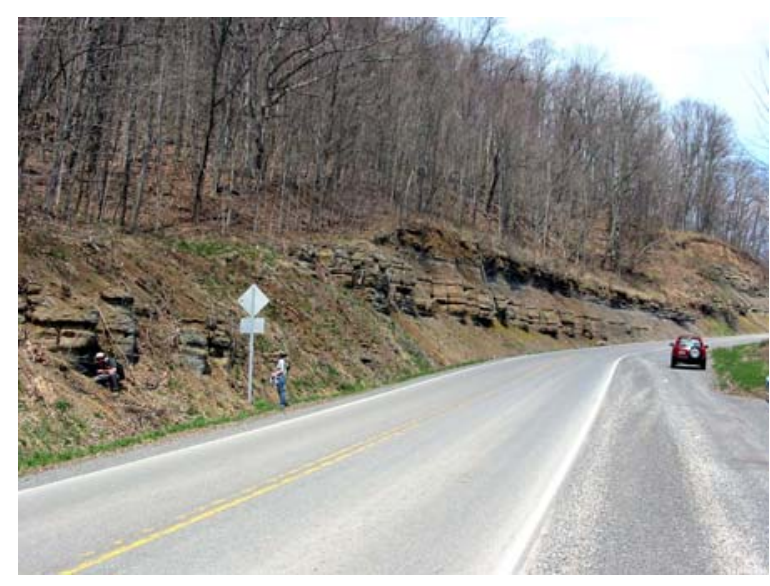

4a. Edray - Base Reynolds

Limestone interval ER- 3- 9.

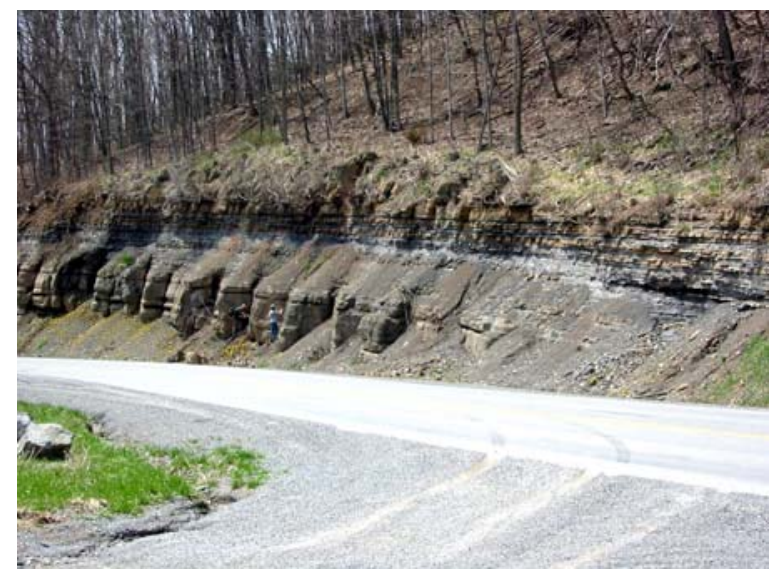

4b. Reynolds Limestone interval at Edray; ER-4-6.

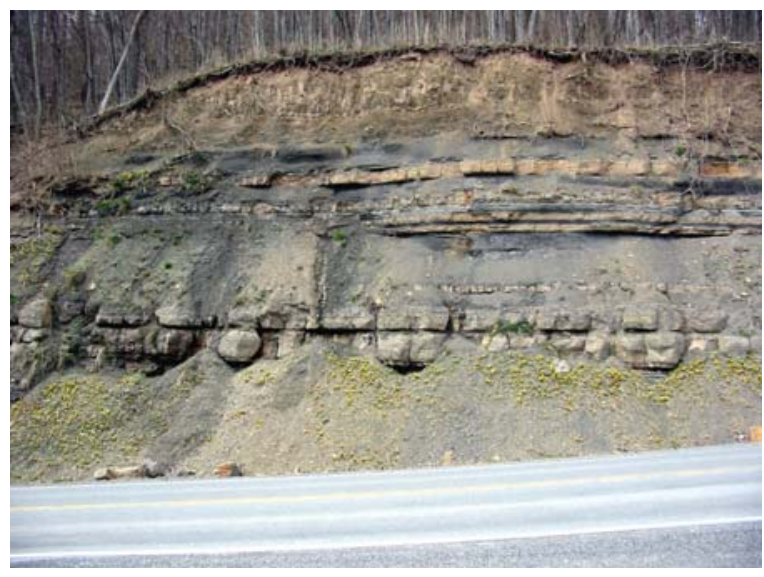

4c. Edray - Reynolds Limestone interval; ER-7- 9. 


\section{Plate 5}

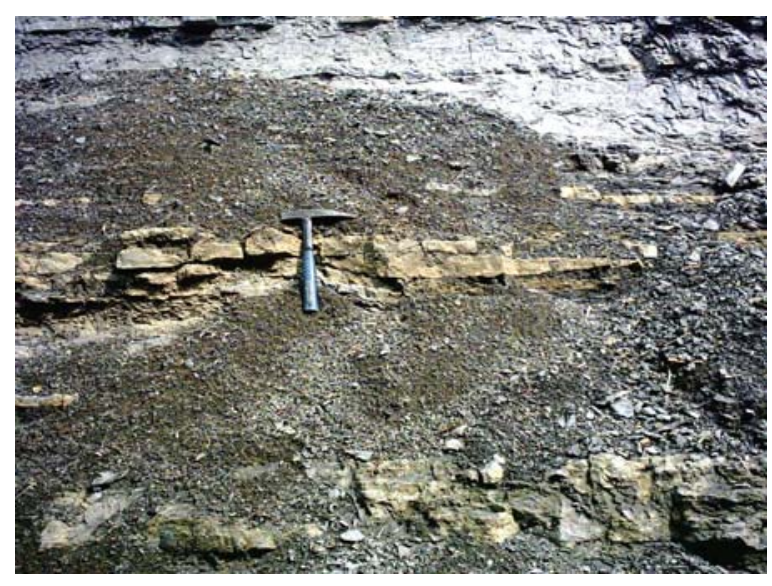

5a. Unit 6 tidal channels at Edray section in the Reynolds Limestone; ER-6

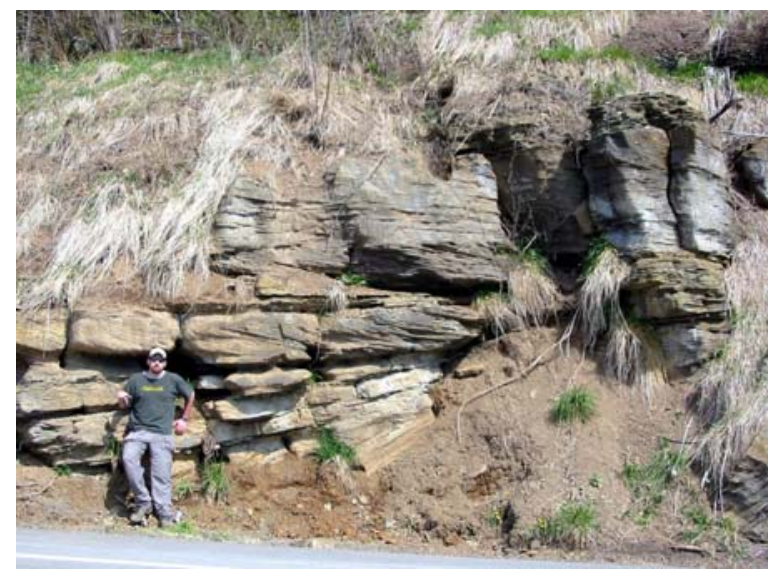

5b. Top Greenbrier Limestone at Droop Mountain section. Note the cross bedding of probable tidal origin; DM-1.

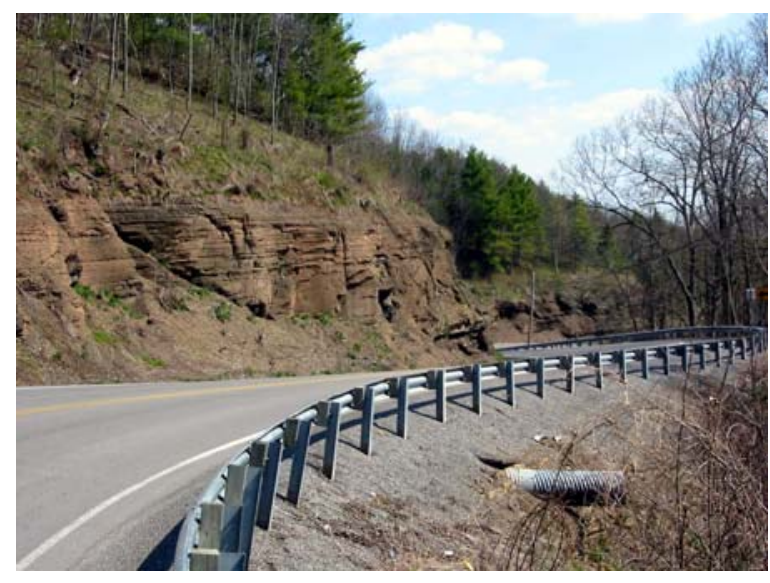

5c. Glenray Limestone at Droop Mountain section; DM-7. 


\section{Plate 6}

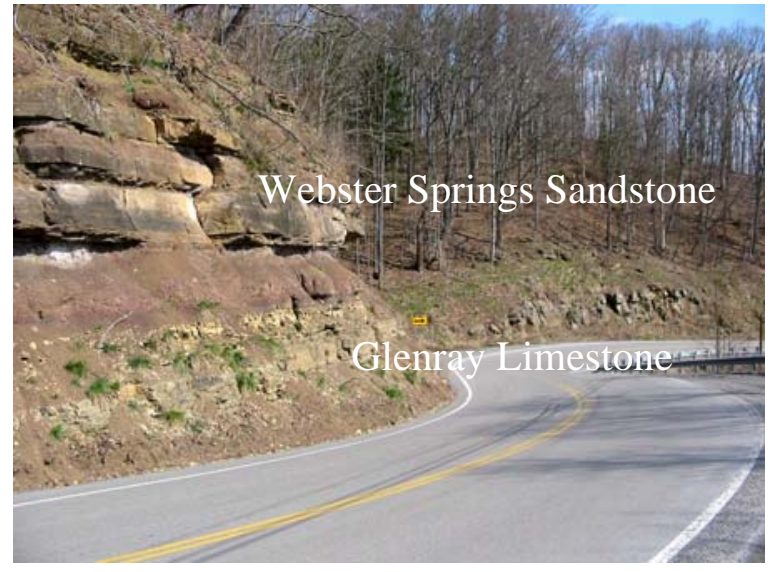

6a. Webster Springs Sandstone and Glenray Limestone at Droop Mountain section; DM-7-10.

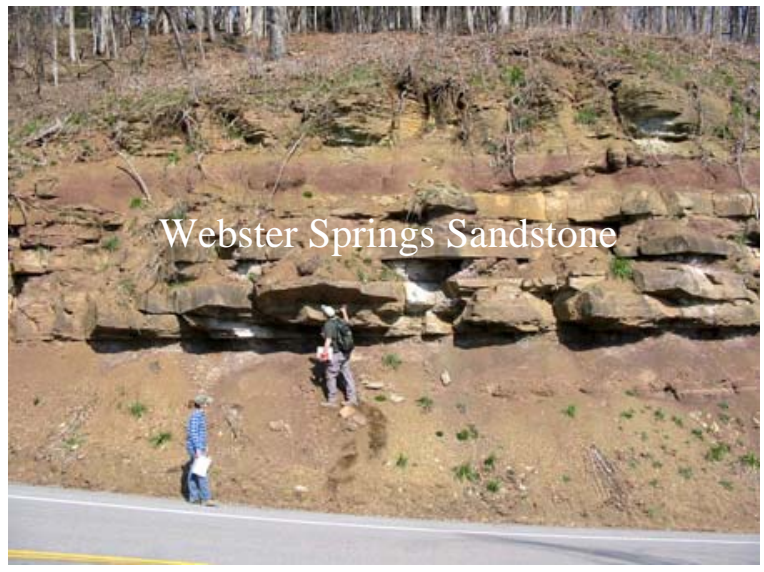

6b. Webster Springs Sandstone at Droop Mountain section; DM-9-

11.

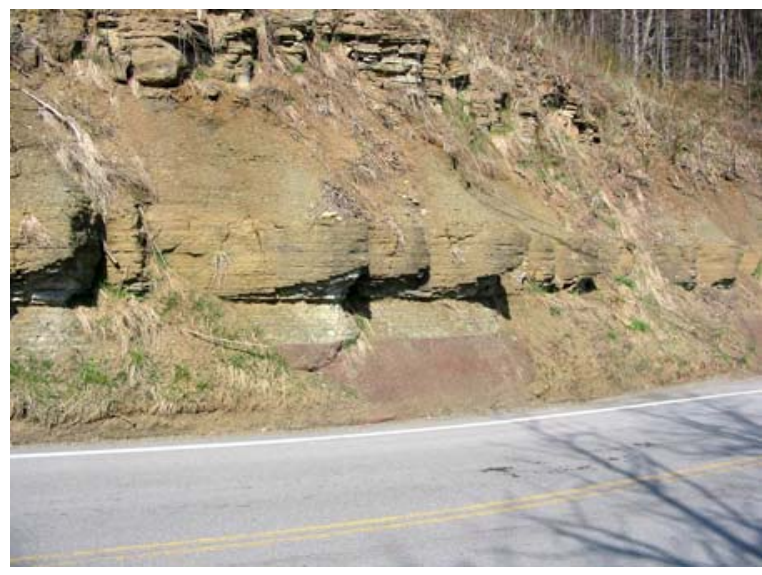

6c. Base of Reynolds

Limestone at Droop Mountain section; DM-11-16. 


\section{Plate 7}

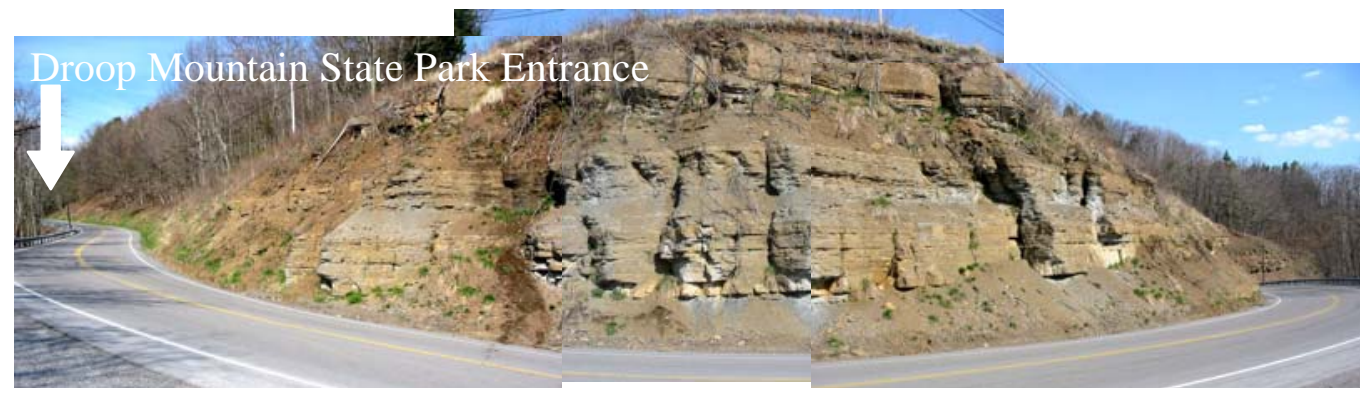

7a. Reynolds Limestone outcrop at Droop Mountain section; DM-11-19. 


\section{GEOLOGIC SETTING}

During the Late Mississippian, much of the Appalachian region was part of a foreland trough (McKinney and Gault, 1989). A paleolatitude of $10^{\circ}-15^{\circ}$ south created a subtropical climate. The generalized paleogeographic maps (Figs. 2, 3) show an elongate northeast-trending marine embayment across central West Virginia (Donaldson and Shumaker, 1981; McKinney and Gault, 1989). The eastern shoreline of the bay typically has a thicker sediment package (Fig.

4) due to the more rapid subsidence near the Appalachian Highlands (Wray and Smosna, 1982). The source area for the clastic sediments of the Mauch Chunk Formation appears to have been the eastern and northeastern Appalachian Highlands (Wray and Smosna, 1982; Brezenski, 1989a,b; McKinney and Gault, 1989).

The Reynolds Limestone is a formal member of the Upper Mississippian, lower Bluefield Formation of the Mauch Chunk Group, in the Chesterian Series, in southern West Virginia (Fig. 5). In northern West Virginia, where the Bluefield Formation is not recognized, the Reynolds Limestone is a member of the Mauch Chunk Formation (Fig. 6). Sediments of the Mauch Chunk Group were deposited during several small-scale transgressive - regressive episodes in the Late Mississippian (Carney and Smosna, 1989). The Mauch Chunk represents a regressive cycle containing two minor transgressive units within the overall lithologic package (Brezenski, 1989a). The first of these transgressive units is the Glenray Limestone; the second is the Reynolds Limestone, the focus of this study.

The definition of the Mauch Chunk varies regionally within the study area (Figs. 1, 6). In southern West Virginia, the Mauch Chunk is an order of magnitude thicker $(100 \mathrm{~s} \mathrm{~m})$ than in northern West Virginia (10s m). As a result, stratigraphic subdivisions recognized in southern West Virginia (Fig. 6) are either absent or unrecognizable in northern West Virginia. The 


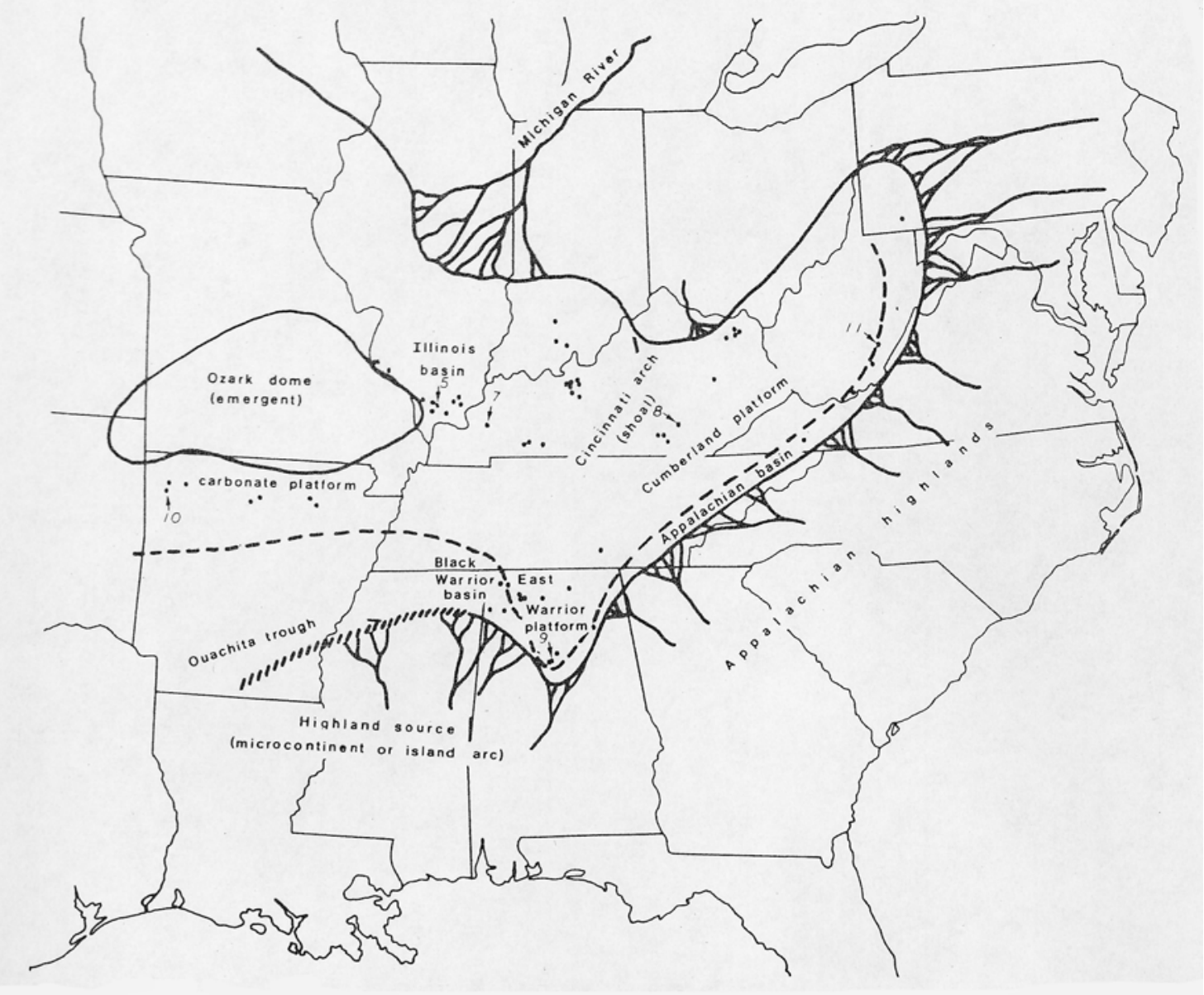

Figure 2. A generalized paleogeographic map showing the elongate northeast trending marine embayment over the central region of West Virginia (McKinney and Gault 1989). 


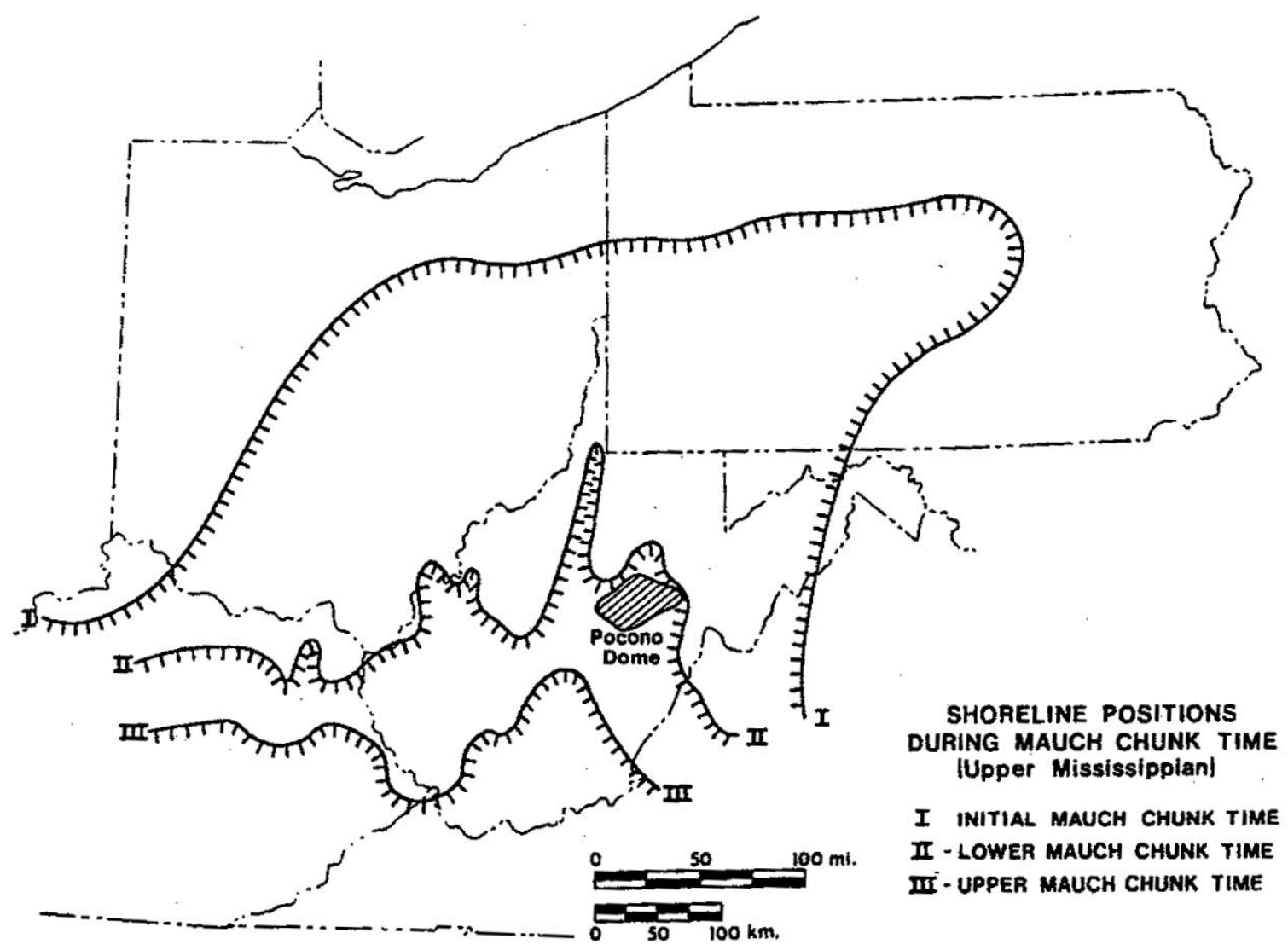

Figure 3. Shoreline positions from time of Greenbrier deposition to Upper Mauch Chunk redbed sedimentation (Donaldson and Shumaker 1981). 


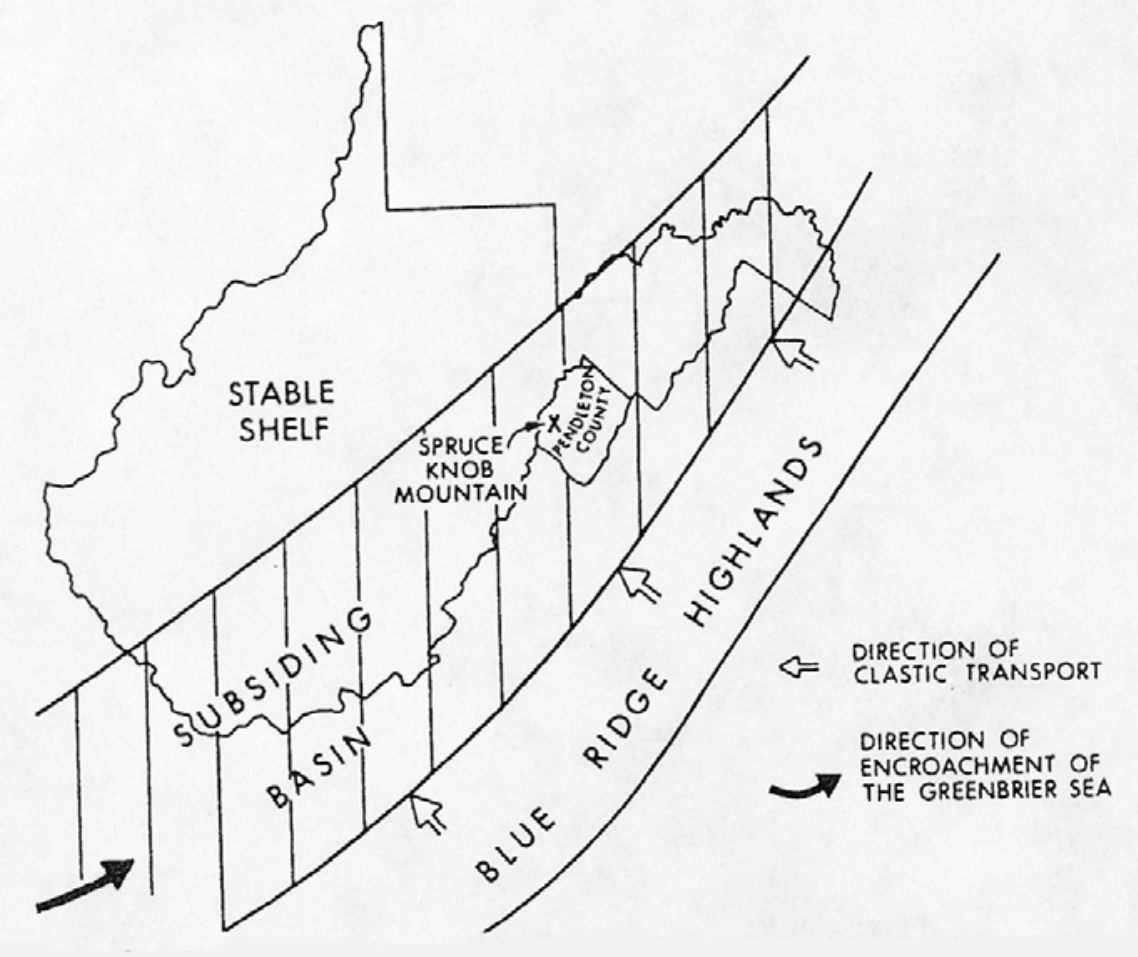

Figure 4. Sedimentation patterns in relation to pre-existing tectonic features of the Appalachian Basin (Wray and Smosna 1982). This figure was originally adapted to show conditions during Greenbrier depositional time. Based on the proximity between the Greenbrier and Reynolds Limestones this model will also represent basinal conditions during Reynolds deposition. 


\begin{tabular}{|c|c|c|c|c|}
\hline \multirow{18}{*}{ 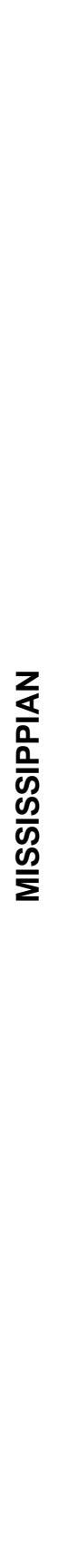 } & \multirow{18}{*}{ 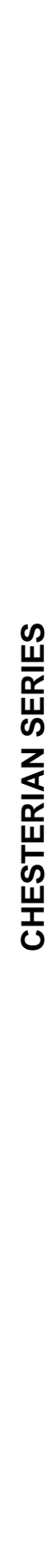 } & \multirow{17}{*}{ 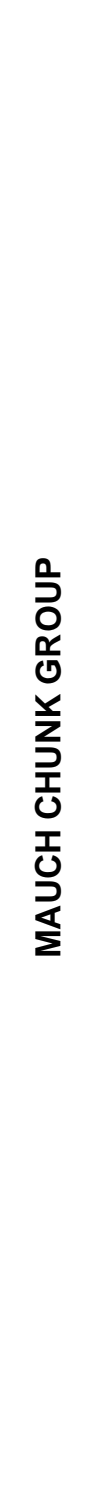 } & \multicolumn{2}{|r|}{ Hinton Formation } \\
\hline & & & \multirow{16}{*}{ 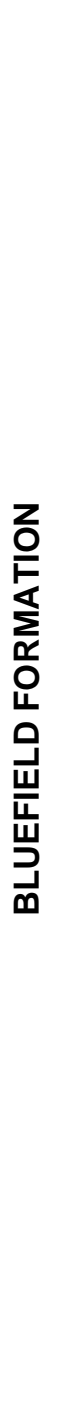 } & Coney Member \\
\hline & & & & Clayton Member \\
\hline & & & & Graham Member \\
\hline & & & & Bertha Member \\
\hline & & & & Bradshaw Member \\
\hline & & & & Indian Mills Member \\
\hline & & & & Raines Corner Member \\
\hline & & & & Possumtrot Shale \\
\hline & & & & Droop Sandstone \\
\hline & & & & Talcott Shale \\
\hline & & & & Ada Shale \\
\hline & & & & Reynolds Limestone \\
\hline & & & & Bickett Shale \\
\hline & & & & Webster Springs Sandstone \\
\hline & & & & Glenray Limestone \\
\hline & & & & Lillydale Shale \\
\hline & & 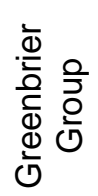 & & Alderson Limestone \\
\hline
\end{tabular}

Figure 5. Stratigraphic position of the Reynolds Limestone Member in the Bluefield Formation in southern West Virginia (Christopher 1992). 


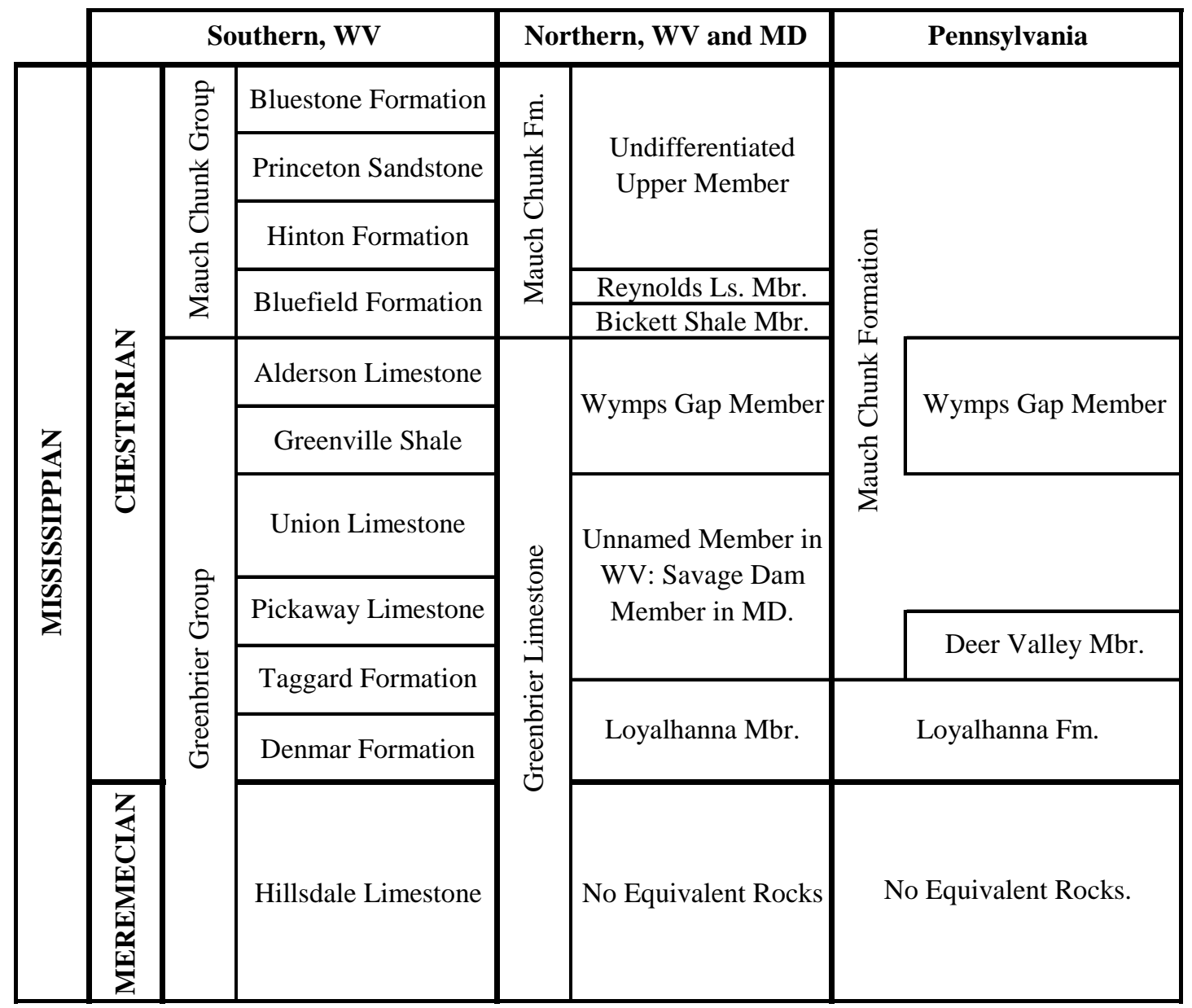

Figure 6. Stratigraphic nomenclature of the Upper Mississippian in northern West Virginia, western Maryland, and southwest Pennsylvania (Arkle et al, 1979; Brezenski, 1989a;

Kammer and Lake, 2001). 
western Maryland section contains the same stratigraphic units as northern West Virginia. The stratigraphic terminology recognized in Pennsylvania is markedly different from both West Virginia and Maryland. The principal difference is the inclusion of much of the Greenbrier Limestone equivalent in the Mauch Chunk Formation of Pennsylvania, except for the Loyalhanna Formation (Fig. 6). Because the top of the Loyalhanna Formation marks the base of the Mauch Chunk Formation in Pennsylvania, the Mauch Chunk section there is much thicker than that of northern West Virginia. In northern West Virginia, the Greenbrier is separated from the Mauch Chunk thus greatly reducing the overall thickness of the Mauch Chunk in comparison to Pennsylvania (Brezenski, 1989b; Lake, 1998).

\section{METHODS OF INVESTIGATION}

\section{Measuring and Describing Outcrop Localities}

The Greenbrier Limestone was used as the lower boundary of the measured stratigraphic interval for all outcrops. Measurement started at the top of the Greenbrier Limestone and continued up-section through the Reynolds Limestone into the overlying non-marine units to ensure that sufficient outcrop was measured to make environmental interpretations about postReynolds depositional conditions. Lithology, sedimentary structures, fossil content, texture, rock color, and rock name were all recorded as part of the description process. Informal units were recognized where changes in lithology, pronounced differences in fossil content, or color were noted.

All measurements were taken in metric units. Depositional and structural dips for the region are very low $\left(0^{\circ}-5^{\circ}\right)$ and were considered to have a negligible effect on the stratigraphic 
thickness. Outcrop localities are either road cuts or quarry high-walls, which allowed measurements to be taken directly on the strata using a cloth tape. Where roadside exposures became too narrow to work safely or outcrop conditions too adverse, alternative measurement techniques were employed. Where strata could not be measured directly, the average slope angle of the ground surface was taken and trigonometric methods were used to calculate true rock thicknesses; this was necessary at the Droop Mountain section.

Rock samples from every measured unit were collected, bagged, labeled, and taken back to the lab for further analysis and reference. Limestone units were sampled more frequently in order to have abundant material for fossil identification and thin-section analysis. In the lab, samples were arranged in stratigraphic order so representative stratigraphic models could be tied directly to field notes.

Fossil identification at both the outcrop and in the lab was conducted through the visual analysis of samples and use of paleontologic literature. This began by compiling a list of all taxa identified in the study interval by Lake (1998). From this, a field identification booklet was organized so outcrop-scale identifications could be made easily. Photographs of each of the taxa were found in the paleontologic literature and scanned into the booklet. References used for each of the various taxa were: brachiopods - Moore (1965a, b), Henry and Gordon (1992), Feldmann et al (1996); bivalves - Clark (1906), Moore (1969a, b), Busans and Hoare (1991), Feldmann et al (1996); gastropods - Moore et al (1952), Feldmann (1996); trilobites - Moore et al (1952), Moore (1959), Feldmann (1996). Laboratory identifications were aided by comparing the samples collected from this study to those cataloged by Lake’s (1998) study.

Field notes were typed and arranged in stratigraphic order and by location from north to south. Detailed stratigraphic sections were drawn using Auto CAD based on the field 
descriptions and hand sample analysis (Appendix 1). Individual outcrops were then combined into a north-south stratigraphic cross-section. Correlations methods are discussed in detail in a later section.

\section{$\underline{\text { Thin Sections }}$}

The research committee set a maximum of 25 thin sections. After fieldwork was completed and detailed stratigraphic columns were constructed, all limestones in the Reynolds Limestone part of section were described and analyzed. From these, a total of 21 thin sections were made. All slides were stained with Alizarin Red S so that calcite (pink) and dolomite (clear) could be differentiated.

Thin sections were examined three times. The first two examinations were performed exclusively for grain identification purposes (Appendix 2). No examination matrices were used and no point counts were made. All identifiable grains were recorded in an Excel spreadsheet and used as a checklist during the actual point-count. The third and final examination of the thin sections was for point-counting. The raw numeric data (Table 2) were converted into percentages (Table 3), in order to show the volume of each grain type identified. During petrologic examination of the thin sections, 28 carbonate and non-carbonate grain constituents were identified (Table 4). Visual analysis of the 300 points and subsequent statistical analysis of the point-count data were used to name the rock type based on Dunham's (1962) classification scheme. A total of 300 points per slide were counted to achieve a statistically valid sample (Harwood, 1988). In order to spread the 300 points over the entire slide, the point count matrix size for each slide was varied. The first point for each slide always 
Table 2. Point Count Raw Number Data. This table lists all points counted during the thin section analysis and the total points counted for each thin section unit

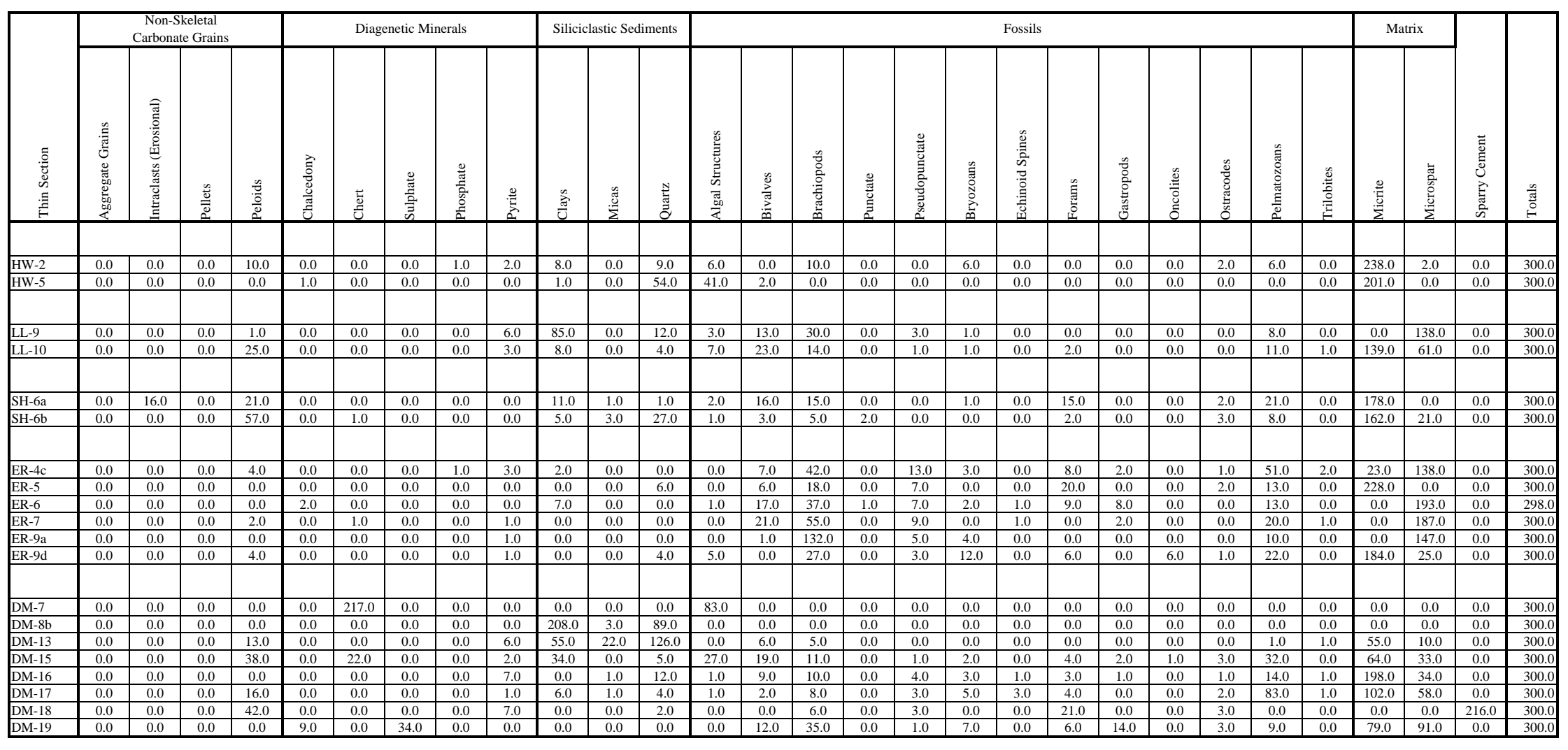


Table 3. Point Count Percent Data. This table normalizes the actual points from Table 2 into a percent of the total sample

\begin{tabular}{|c|c|c|c|c|c|c|c|c|c|c|c|c|c|c|c|c|c|c|c|c|c|c|c|c|c|c|c|c|c|}
\hline \multirow[b]{2}{*}{ 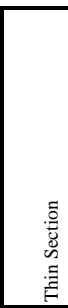 } & \multicolumn{4}{|c|}{$\begin{array}{c}\text { Non-Skeletal } \\
\text { Carbonate Grains }\end{array}$} & \multicolumn{5}{|c|}{ Diagenetic Minerals } & \multicolumn{3}{|c|}{ Siliciclastic Sediments } & \multicolumn{13}{|c|}{ Fossils } & \multicolumn{2}{|c|}{ Matrix } & \multirow[b]{2}{*}{ 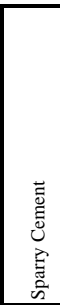 } & \multirow[b]{2}{*}{ 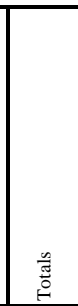 } \\
\hline & 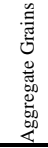 & 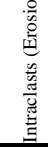 & $\frac{\mathscr{0}}{\bar{\Xi}}$ & $\begin{array}{l}\frac{n}{0} \\
\frac{0}{0} \\
0\end{array}$ & $\begin{array}{l}\frac{\vec{E}}{\tilde{E}} \\
\frac{\tilde{g}}{\tilde{n}} \\
\end{array}$ & 莺 & 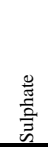 & 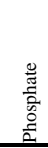 & 尝 & 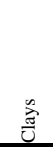 & 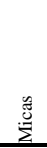 & 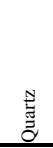 & 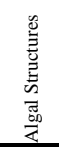 & 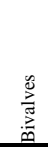 & 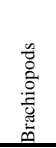 & 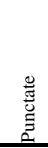 & 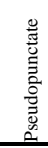 & 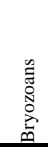 & 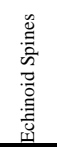 & 呢 & 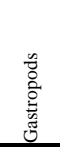 & 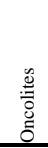 & 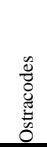 & 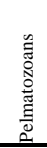 & 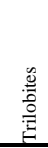 & 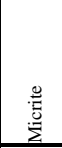 & 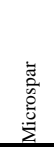 & & \\
\hline \begin{tabular}{|l|}
$\mathrm{HW}-2$ \\
$\mathrm{HW}-5$
\end{tabular} & 0.0 & 0.0 & 0.0 & 3.3 & 0.0 & 0.0 & 0.0 & 0.3 & 0.7 & 2.7 & 0.0 & 3.0 & 2.0 & 0.0 & 3.3 & 0.0 & 0.0 & 2.0 & 0.0 & 0.0 & 0.0 & 0.0 & 0.7 & 2.0 & 0.0 & \begin{tabular}{|l|}
79.3 \\
\end{tabular} & 0.7 & 0.0 & 100.0 \\
\hline HW-5 & 0.0 & $\begin{array}{l}0.0 \\
\end{array}$ & 0.0 & $\begin{array}{l}0.0 \\
\end{array}$ & 0.3 & $\begin{array}{l}0.0 \\
\end{array}$ & 0.0 & $\begin{array}{l}0.0 \\
\end{array}$ & 0.0 & 0.3 & 0.0 & 18.0 & 13.7 & $\begin{array}{l}0.7 \\
\end{array}$ & 0.0 & $\begin{array}{l}0.0 \\
\end{array}$ & 0.0 & $\begin{array}{l}0.0 \\
\end{array}$ & 0.0 & $\begin{array}{l}0.0 \\
\end{array}$ & 0.0 & 0.0 & 0.0 & 0.0 & 0.0 & \begin{tabular}{|l|l}
67.0 \\
\end{tabular} & $\begin{array}{l}0.0 \\
\end{array}$ & 0.0 & 100.0 \\
\hline LL-9 & 0.0 & $\begin{array}{l}0.0 \\
\end{array}$ & 0.0 & \begin{tabular}{|l|}
0.3 \\
\end{tabular} & 0.0 & 0.0 & 0.0 & \begin{tabular}{|l|l}
0.0 \\
\end{tabular} & 2.0 & 28.3 & 0.0 & 4.0 & 1.0 & 4.3 & 10.0 & 0.0 & $\begin{array}{l}1.0 \\
\end{array}$ & \begin{tabular}{|l|}
0.3 \\
\end{tabular} & 0.0 & $\begin{array}{l}0.0 \\
\end{array}$ & \begin{tabular}{|l|}
0.0 \\
\end{tabular} & 0.0 & 0.0 & 2.7 & $\begin{array}{l}0.0 \\
\end{array}$ & \begin{tabular}{|l|l|}
0.0 \\
\end{tabular} & 45.9 & 0.0 & $\begin{array}{l}100.0 \\
1000\end{array}$ \\
\hline \begin{tabular}{|l} 
LL-10 \\
\end{tabular} & $\begin{array}{l}0.0 \\
\end{array}$ & $\begin{array}{l}0.0 \\
\end{array}$ & $\begin{array}{l}0.0 \\
\end{array}$ & $\begin{array}{l}8.3 \\
\end{array}$ & $\begin{array}{l}0.0 \\
\end{array}$ & $\begin{array}{l}0.0 \\
\end{array}$ & $\begin{array}{l}0.0 \\
\end{array}$ & 0.0 & 1.0 & $\begin{array}{l}2.7 \\
\end{array}$ & 0.0 & 1.3 & $\begin{array}{l}2.3 \\
\end{array}$ & $\begin{array}{l}7.7 \\
\end{array}$ & 4.7 & $\begin{array}{l}0.0 \\
\end{array}$ & $\begin{array}{l}0.3 \\
\end{array}$ & $\begin{array}{l}0.3 \\
\end{array}$ & 0.0 & $\begin{array}{l}0.7 \\
\end{array}$ & 0.0 & $\begin{array}{l}0.0 \\
\end{array}$ & $\begin{array}{l}0.0 \\
\end{array}$ & 3.7 & $\begin{array}{l}0.3 \\
\end{array}$ & \begin{tabular}{|r|}
46.3 \\
\end{tabular} & 20.3 & 0.0 & 100.0 \\
\hline \begin{tabular}{|l|} 
SH-6a \\
SH-6h
\end{tabular} & $\begin{array}{l}0.0 \\
0.0\end{array}$ & 5.3 & $\begin{array}{l}0.0 \\
0.0\end{array}$ & $\begin{array}{r}7.0 \\
190\end{array}$ & $\begin{array}{l}0.0 \\
0.0\end{array}$ & $\begin{array}{l}0.0 \\
0.3\end{array}$ & $\begin{array}{l}0.0 \\
0.0\end{array}$ & 0.0 & $\begin{array}{l}0.0 \\
0.0\end{array}$ & $\begin{array}{l}3.7 \\
17\end{array}$ & 0.3 & $\begin{array}{l}0.3 \\
90\end{array}$ & \begin{tabular}{|l|}
0.7 \\
03
\end{tabular} & 5.3 & $\begin{array}{l}5.0 \\
17\end{array}$ & 0.0 & 0.0 & 0.3 & $\begin{array}{l}0.0 \\
0.0\end{array}$ & 5.0 & $\begin{array}{l}0.0 \\
0.0\end{array}$ & $\begin{array}{l}0.0 \\
0.0\end{array}$ & $\begin{array}{l}0.7 \\
10\end{array}$ & $\begin{array}{l}7.0 \\
27\end{array}$ & 0.0 & 59.3 & $\begin{array}{l}0.0 \\
70\end{array}$ & 0.0 & 100.0 \\
\hline & & & & & & & & & & & & & & & & & & & & & & & & & & & & & \\
\hline ER-4c & 0.0 & 0.0 & 0.0 & 1.3 & 0.0 & 0.0 & 0.0 & 0.3 & 1.0 & 0.7 & 0.0 & 0.0 & 0.0 & 2.3 & 14.0 & 0.0 & 4.3 & 1.0 & 0.0 & 2.7 & $\begin{array}{l}0.7 \\
\end{array}$ & 0.0 & 0.3 & 17.0 & 0.7 & 7.7 & 46.0 & 0.0 & 100.0 \\
\hline ER-5 & $\begin{array}{l}0.0 \\
\end{array}$ & $\begin{array}{l}0.0 \\
\end{array}$ & 0.0 & $\begin{array}{l}0.0 \\
\end{array}$ & 0.0 & 0.0 & 0.0 & $\begin{array}{l}0.0 \\
\end{array}$ & 0.0 & 0 & 00 & 2.0 & 0.0 & 2.0 & 6.0 & 0.0 & $\begin{array}{l}2.3 \\
\end{array}$ & 0.0 & 0.0 & 6.7 & 0.0 & 0.0 & $\begin{array}{l}0.7 \\
\end{array}$ & 4.3 & 0.0 & 75.9 & 0.0 & 0.0 & 100.0 \\
\hline \begin{tabular}{|l} 
ER-6 \\
\end{tabular} & 0. & 0 & $\begin{array}{l}0.0 \\
\end{array}$ & \begin{tabular}{|l|l}
0.0 \\
\end{tabular} & 0 & 0 & 0 & 0. & 0 & & & 0.0 & 0 & $\begin{array}{l}.0 .7 \\
\end{array}$ & 12.4 & \begin{tabular}{|l|}
0.3 \\
\end{tabular} & \begin{tabular}{|l|}
2.3 \\
\end{tabular} & 0.07 & & 3.0 & $\begin{array}{l}2.7 \\
\end{array}$ & 0 & 0.0 & $\begin{array}{l}.4 \\
\end{array}$ & 0 & 0.0 & 64.7 & 0.0 & 100.0 \\
\hline \begin{tabular}{|l} 
ER-7 \\
\end{tabular} & 0. & 0 & $\begin{array}{l}0.0 \\
\end{array}$ & \begin{tabular}{|l}
0.7 \\
0.7
\end{tabular} & 0 & 0 & 0 & $\begin{array}{l}0.0 \\
\end{array}$ & $\begin{array}{l}0.3 \\
\end{array}$ & & 0.0 & 0.0 & 0 & $\begin{array}{l}.87 \\
7.0\end{array}$ & 18.3 & $\begin{array}{l}0.0 \\
0.0\end{array}$ & $\begin{array}{l}. .0 \\
\end{array}$ & $\begin{array}{l}0.0 \\
\end{array}$ & & 0.0 & 0 & 0.0 & 0.0 & 1.7 & 0 & $\begin{array}{l}0.0 \\
\end{array}$ & $\begin{array}{l}62.3 \\
62.3\end{array}$ & $\begin{array}{l}0.0 \\
0.0\end{array}$ & \begin{tabular}{c|}
100.0 \\
\end{tabular} \\
\hline ER-9a & $\begin{array}{l}0.0 \\
0.0\end{array}$ & $\begin{array}{l}0.0 \\
0.0\end{array}$ & $\begin{array}{l}0.0 \\
0.0\end{array}$ & $\begin{array}{l}0.1 \\
0.0\end{array}$ & $\begin{array}{l}0.0 \\
0.0\end{array}$ & $\begin{array}{l}0.3 \\
0.0\end{array}$ & $\begin{array}{l}0.0 \\
0.0\end{array}$ & $\begin{array}{l}0.0 \\
0.0\end{array}$ & \begin{tabular}{|l}
0.3 \\
0.3
\end{tabular} & 0.0 & 0 & 0.0 & 0.0 & $\begin{array}{l}0.0 \\
0.3\end{array}$ & $\begin{array}{l}18.3 \\
44.0\end{array}$ & $\begin{array}{l}0.0 \\
0.0\end{array}$ & $\begin{array}{l}.00 \\
1.7\end{array}$ & $\begin{array}{l}0.0 \\
1.3\end{array}$ & 0.0 & 0.0 & 0.0 & $\begin{array}{l}0.0 \\
0.0\end{array}$ & 0.0 & $\begin{array}{l}.1 \\
3.3 \\
\end{array}$ & $\begin{array}{l}0.3 \\
0.0\end{array}$ & $\begin{array}{l}0.0 \\
0.0\end{array}$ & $\begin{array}{l}\frac{82.3}{49.0} \\
\end{array}$ & $\begin{array}{l}0.0 \\
0.0\end{array}$ & $\begin{array}{l}1000.0 \\
\end{array}$ \\
\hline ER-9d & $\begin{array}{l}0.0 \\
\end{array}$ & $\begin{array}{l}0.0 \\
\end{array}$ & $\begin{array}{l}0.0 \\
\end{array}$ & $\begin{array}{l}1.3 \\
\end{array}$ & $\begin{array}{l}0.0 \\
\end{array}$ & $\begin{array}{l}0.0 \\
\end{array}$ & $\begin{array}{l}0.0 \\
\end{array}$ & $\begin{array}{l}0.0 \\
\end{array}$ & $\begin{array}{l}0.3 \\
\end{array}$ & 0.0 & $\begin{array}{l}0.0 \\
\end{array}$ & 1.3 & \begin{tabular}{|l|l|}
1.7 \\
\end{tabular} & $\begin{array}{l}0.0 \\
\end{array}$ & $\begin{array}{l}9.0 \\
\end{array}$ & $\begin{array}{l}0.0 \\
\end{array}$ & $\begin{array}{l}1.0 \\
\end{array}$ & $\begin{array}{l}4.0 \\
\end{array}$ & $\begin{array}{l}0.0 \\
\end{array}$ & $\begin{array}{l}2.0 \\
\end{array}$ & $\begin{array}{l}0.0 \\
\end{array}$ & $\begin{array}{l}2.0 \\
\end{array}$ & $\begin{array}{l}0.3 \\
\end{array}$ & $\begin{array}{l}7.3 \\
\end{array}$ & $\begin{array}{l}0.0 \\
\end{array}$ & 61.3 & 8.3 & 0.0 & 100.0 \\
\hline DM-7 & 0.0 & 0.0 & 0.0 & 0.0 & 0. & 72.3 & 0. & 0.0 & 0.0 & 0 & 0.0 & 0. & 27.7 & 0.0 & 0.0 & 0.0 & 0.0 & 0.0 & $\overline{0.0}$ & 0.0 & 0.0 & 0.0 & 0.0 & 0.0 & 0.0 & 0.0 & 0.0 & 0.0 & 100.0 \\
\hline & 0. & 0 & 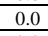 & 0. & 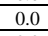 & 0 & 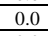 & 0 & 0. & & 1. & 25 & 0. & 0.0 & 0. & 0.0 & 0.0 & & & 0 & & 0 & 0.0 & & & 0 & 0.0 & 0.0 & 10 \\
\hline & 0. & 0 & 0.0 & 4.3 & 0 & 0.0 & 0. & 18 & 7 & 0.0 & 2. & 42 & 0 & 2.0 & 1.7 & 0 & 0. & 0 & 0 & 0. & 0 & 0 & 0.0 & 0 & & 18.3 & 3.3 & 0.0 & 100.0 \\
\hline & 0. & 0 & 0.0 & 12.7 & 0 & 7.3 & 0.0 & 0 & 0 & 11 & 0.0 & 1. & 9 & 6.3 & 3.7 & 0 & 0. & 0 & 0 & 1. & 0 & 0 & 1.0 & 10 & & 21.3 & 11.0 & 0.0 & 100.0 \\
\hline & 0.0 & 0.0 & 0.0 & 0.0 & 0.0 & 0.0 & 0.0 & 0.0 & 0.3 & 0.0 & 2.3 & 4.0 & 0.3 & 3.0 & 3.3 & 0.0 & 1. & 1.0 & 0.3 & 1.0 & 0 & 0 & 0.3 & 4 & 0 & 66.0 & 11.3 & 0.0 & 100.0 \\
\hline DM- & 0.0 & 0.0 & 0.0 & 5.3 & 0.0 & 0.0 & 0.0 & 0.0 & 0.3 & 2.0 & 0.3 & 1.3 & 0.3 & 0.7 & 2.7 & 0.0 & 1.0 & 1.7 & 1.0 & 1.3 & 0 & 0.0 & 0.7 & 27.6 & 0.3 & \begin{tabular}{|l|l}
34.0 \\
\end{tabular} & 19.3 & 0.0 & 100.0 \\
\hline & 0.0 & 0. & 0.0 & 14.0 & 0.0 & 0.0 & 0.0 & 0.0 & 2.3 & 0.0 & 0.0 & 0.7 & 0.0 & 0.0 & 2.0 & 0.0 & 1.0 & 0.0 & 0.0 & 7.0 & 0. & 0.0 & 1.0 & 0.0 & 0.0 & 0.0 & 0.0 & 72.0 & 100.0 \\
\hline $\mid \frac{19}{D M-19}$ & 0.0 & 0.0 & 0.0 & $\frac{1.0}{0.0}$ & 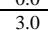 & 0.0 & $\begin{array}{l}11.3 \\
11.3\end{array}$ & 0.0 & $\begin{array}{l}2 . .5 \\
0.0\end{array}$ & 0.0 & 0.0 & 0.0 & $\begin{array}{l}0.0 \\
0.0\end{array}$ & $\begin{array}{l}0.0 \\
4.0\end{array}$ & $\begin{array}{l}2.0 \\
11.7\end{array}$ & 0.0 & $\begin{array}{l}1.0 \\
0.3\end{array}$ & $\begin{array}{l}0.0 \\
2.3\end{array}$ & $\frac{0.0}{0.0}$ & 2.0 & 0.0 & 0.0 & 1.0 & 3.0 & 0.0 & 26.3 & 30.3 & 0.0 & 100.0 \\
\hline
\end{tabular}


Table 4. Observational Data for Thin Section Units. This table reports the data collected for thin section units. Fossil content is based on how previlant fossil material was in the unit being studied. Outcrop scale identifications are: diversity, which refers to the number of generic level identifications that were made in the field, and sedimentary structures.

\begin{tabular}{|c|c|c|c|c|c|c|c|}
\hline Location & Siliciclastic Sediment & $\begin{array}{c}\text { Siliciclastic } \\
\text { Sediments (\%) } \\
\end{array}$ & Fossil Content & $\begin{array}{l}\text { Diversity } \\
(\# \text { Taxa) }\end{array}$ & Algal Structures (\%) & Bivalves (\%) & Brachiopods (\%) \\
\hline $\mathrm{HW}-2$ & Little & 5.70 & Rare & 2 & 2.00 & 0.00 & 3.33 \\
\hline $\mathrm{HW}-5$ & Moderate & 18.39 & Rare & 2 & 13.67 & 0.67 & 0.00 \\
\hline LL-9 & Moderate & 32.96 & Common & 10 & 1.00 & 4.32 & 11.01 \\
\hline LL-10 & Little & 4.03 & Common & 10 & 2.33 & 7.65 & 4.99 \\
\hline SH-6a & Little & 4.33 & Common & 3 & 0.67 & 5.33 & 5.00 \\
\hline SH-6b & Moderate & 11.70 & Common & 3 & 0.33 & 1.00 & 2.33 \\
\hline ER-4c & Little & 0.67 & Common & 5 & 0.00 & 2.33 & 18.33 \\
\hline ER-5 & Little & 2.03 & Common & 3 & 0.00 & 2.00 & 8.32 \\
\hline ER-6 & Little & 2.36 & Abundant & 9 & 0.34 & 5.70 & 15.09 \\
\hline ER-7 & $\mathrm{N} / \mathrm{A}$ & 0.00 & Abundant & 6 & 0.03 & 6.99 & 21.30 \\
\hline ER-9a & Little & 0.03 & Abundant & 3 & 0.00 & 0.33 & 45.65 \\
\hline ER-9d & Little & 1.37 & Rare & 2 & 1.67 & 0.00 & 9.99 \\
\hline DM-7 & $\mathrm{N} / \mathrm{A}$ & 0.00 & Rare & 5 & 27.67 & 0.00 & 0.00 \\
\hline DM-8b & High & 100.00 & Rare & 0 & 0.00 & 0.00 & 0.00 \\
\hline DM-13 & High & 69.05 & Rare & 3 & 0.00 & 2.00 & 1.67 \\
\hline DM-15 & Moderate & 14.13 & Abundant & 6 & 9.00 & 6.33 & 4.00 \\
\hline DM-16 & Little & 4.44 & Common & 7 & 0.33 & 3.00 & 4.67 \\
\hline DM-17 & Little & 3.68 & Common & 8 & 0.33 & 0.67 & 3.70 \\
\hline DM-18 & Little & 0.68 & Rare & 6 & 0.00 & 0.00 & 3.00 \\
\hline DM-19 & $\mathrm{N} / \mathrm{A}$ & 0.00 & Abundant & 4 & 0.00 & 4.00 & 11.99 \\
\hline
\end{tabular}




\begin{tabular}{|c|c|c|c|c|c|c|c|c|}
\hline Location & Brachiopods (\%) & Bryozoans (\%) & Echinoid Spines (\%) & Forams (\%) & Gastropods (\%) & Oncolites (\%) & Ostacodes (\%) & $\begin{array}{c}\text { Pelmatozoan Debris } \\
(\%)\end{array}$ \\
\hline $\mathrm{HW}-2$ & 3.33 & 2.00 & 0.00 & 0.00 & 0.00 & 0.00 & 0.67 & 2.00 \\
\hline LL-9 & 11.01 & 0.33 & 0.03 & 0.03 & 0.00 & 0.00 & 0.00 & 2.66 \\
\hline LL-10 & 4.99 & 0.33 & 0.03 & 0.67 & 0.00 & 0.00 & 0.03 & 3.66 \\
\hline SH-6b & 2.33 & 0.00 & 0.00 & 0.67 & 0.03 & 0.00 & 1.00 & 2.66 \\
\hline ER-4c & 18.33 & 1.00 & 0.03 & 2.67 & 0.67 & 0.00 & 0.33 & 16.99 \\
\hline ER-5 & 8.32 & 0.00 & 0.03 & 6.66 & 0.00 & 0.00 & 0.67 & 4.33 \\
\hline ER-6 & 15.09 & 0.67 & 0.34 & 3.02 & 2.68 & 0.00 & 0.03 & 4.36 \\
\hline DM-7 & 0.00 & 0.00 & 0.00 & 0.00 & 0.00 & 0.00 & 0.00 & 0.00 \\
\hline DM-8b & 0.00 & 0.00 & 0.00 & 0.00 & 0.00 & 0.00 & 0.00 & 0.00 \\
\hline DM-13 & 1.67 & 0.00 & 0.00 & 0.00 & 0.00 & 0.00 & 0.00 & 0.33 \\
\hline DM-15 & 4.00 & 0.67 & 0.03 & 1.33 & 0.67 & 0.33 & 1.00 & 10.66 \\
\hline DM-16 & 4.67 & 1.00 & 0.33 & 1.00 & 0.33 & 0.00 & 0.33 & 4.67 \\
\hline DM-17 & 3.70 & 1.67 & 1.00 & 1.33 & 0.03 & 0.00 & 0.67 & 27.64 \\
\hline DM-18 & 3.00 & 0.00 & 0.03 & 7.00 & 0.00 & 0.00 & 1.00 & 0.03 \\
\hline DM-19 & 11.99 & 2.33 & 0.03 & 2.00 & 4.66 & 0.00 & 1.00 & 3.00 \\
\hline
\end{tabular}




\begin{tabular}{|c|c|c|c|c|c|c|c|c|c|}
\hline Location & Trilobites (\%) & Fossil Grains (\%) & $\begin{array}{l}\text { Sedimentary } \\
\text { Structures }\end{array}$ & $\begin{array}{l}\text { Early Diagenetic } \\
\text { Processes }\end{array}$ & Micrite & $\begin{array}{l}\overline{\text { Iatrix - Type }} \\
\text { Microspar }\end{array}$ & Sparry Cement & $\begin{array}{c}\text { Texture } \\
\text { Mud or Grain } \\
\text { Supported }\end{array}$ & Rock Name \\
\hline HW-2 & 0.00 & 10.00 & Laminae & Pyrite, Cementation & 79.87 & 0.67 & 0.00 & Mud & Wackestone \\
\hline HW-5 & 0.00 & 14.33 & $\begin{array}{l}\text { Hummocky Cross } \\
\text { Stratification }\end{array}$ & Pyrite & 67.22 & 0.00 & 0.00 & Mud & $\begin{array}{l}\text { Argillaceous } \\
\text { Wackestone }\end{array}$ \\
\hline LL-9 & 0.03 & 19.42 & $\mathrm{~N} / \mathrm{A}$ & Pyrite & 0.00 & 46.84 & 0.00 & Grain & Packstone \\
\hline LL-10 & 0.33 & 20.03 & Laminae & Pyrite, Cementation & 46.74 & 20.51 & 0.00 & Mud & Skeletal-Wackestone \\
\hline SH-6a & 0.03 & 24.05 & $\mathrm{~N} / \mathrm{A}$ & Cementation & 59.29 & 0.00 & 0.00 & Mud & Skeletal-Wackestone \\
\hline SH-6b & 0.00 & 8.03 & $\mathrm{~N} / \mathrm{A}$ & Cementation & 54.14 & 7.02 & 0.00 & Mud & Peloidal Mudstone \\
\hline ER-4c & 0.67 & 43.02 & $\mathrm{~N} / \mathrm{A}$ & Pyrite, Cementation & 7.74 & 46.45 & 0.00 & Mud & Skeletal-Wackestone \\
\hline ER-5 & 0.00 & 22.00 & $\begin{array}{l}\text { Hummocky Cross } \\
\text { Stratification, X-beds }\end{array}$ & Pyrite & 75.92 & 0.00 & 0.00 & Mud & Skeletal-Wackestone \\
\hline ER-6 & 0.03 & 32.25 & $\begin{array}{l}\text { Channel Fills, X-beds, } \\
\text { Ripples }\end{array}$ & $\mathrm{N} / \mathrm{A}$ & 0.00 & 65.16 & 0.00 & Mud & \begin{tabular}{|l|} 
Skeletal- \\
Wackst/Packst
\end{tabular} \\
\hline ER-7 & 0.33 & 36.38 & Bioturbation & Pyrite, Cementation & 0.00 & 62.69 & 0.00 & Grain & Skeletal Packstone \\
\hline ER-9a & 0.00 & 50.65 & N / A & Pyrite, Cementation & 0.00 & 49.15 & 0.00 & Grain & Packstone \\
\hline ER-9d & 0.03 & 27.37 & $\mathrm{~N} / \mathrm{A}$ & Pyrite, Cementation & 61.48 & 8.35 & 0.00 & Mud & Skeletal-Wackestone \\
\hline DM-7 & 0.00 & 27.67 & Algal Laminae & $\mathrm{N} / \mathrm{A}$ & 0.00 & 0.00 & 0.00 & $\mathrm{~N} / \mathrm{A}$ & Algal Boundstone \\
\hline DM-8b & 0.00 & 0.00 & Microlaminae, Micro- & Pyrite & 0.00 & 0.00 & 0.00 & Grain & Calcareous Siltstone \\
\hline DM-13 & 0.33 & 4.33 & Laminae & Pyrite, Cementation & 18.71 & 3.40 & 0.00 & Grain & Calcareous Siltstone \\
\hline DM-15 & 0.00 & 34.02 & $\mathrm{~N} / \mathrm{A}$ & Pyrite, Cementation & 23.18 & 11.95 & 0.00 & Mud & \begin{tabular}{|l} 
Argillaceous Skeletal- \\
Wackestone \\
\end{tabular} \\
\hline DM-16 & 0.33 & 16.00 & $\mathrm{~N} / \mathrm{A}$ & Pyrite & 67.58 & 11.60 & 0.00 & Mud & Wackestone \\
\hline DM-17 & 0.33 & 37.36 & $\mathrm{~N} / \mathrm{A}$ & Pyrite, Cementation & 34.08 & 19.38 & 0.00 & Grain & Skeletal-Packstone \\
\hline DM-18 & 0.00 & 11.06 & $\mathrm{~N} / \mathrm{A}$ & Pyrite, Cementation & 0.00 & 0.00 & 73.67 & Grain & Peloidal Packstone \\
\hline DM-19 & 0.00 & 29.00 & $\mathrm{~N} / \mathrm{A}$ & Pyrite, Cementation & 30.72 & 35.38 & 0.00 & Mud & Skeletal-Wackestone \\
\hline
\end{tabular}


started at the lower left corner of the slide. The count continued slide right recording the points directly below the cross hairs at the pre-determined matrix interval. Points were recorded on slide counters and transferred into Excel spreadsheets for further analysis.

\section{LITHOLOGIC DESCRIPTIONS}

\section{Introduction}

Lithologic descriptions are arranged in stratigraphic order from the bottom upward. There are three major divisions in the outcrops that allow the descriptions generally to be grouped together. The first of these is the Lower Clastic Unit, which spans from the top of the Greenbrier Limestone to the base of the Reynolds Limestone. The second group - the Middle Carbonate Units - include the Reynolds and Glenray Limestone intervals. The third, and uppermost group, the Upper Clastic Units encompass all the non-carbonate units above the top of the Reynolds Limestone.

The carbonates are described based on Dunham's (1962) classification scheme - beginning with mudtones. All units with thin-sections (Tables 2, 3, 4) are denoted with asterisks (ex. HW2*). All clastic lithofacies were described based on grain size, sorting, angularity of grains, fossils, color, and sedimentary structures. Dunham's system is based on the ratio between the amount of matrix (lime mud) and carbonate grains, that is, a mud-supported or grain-supported fabric. Constituents from the point-count data that aid in the interpretation of the rock name include: non-skeletal carbonate grains, diagenetic non-carbonate grains, siliciclastic sediments (quartz, micas, clays), fossil grains, and matrix (micrite, microspar) or sparry cement. All data are reported as a percent of the total sample, (Tables 4, 5) and where multiple units are identified the values will be the average of those units (Tables 6, 7). 
Table 5. These are point count percent category totals condensed from Table 3.

\begin{tabular}{|c|c|c|c|c|c|c|c|}
\hline & Non-Skel & Diagenetic & Siliciclastic & Fossil & Micrite & Mic Spar & Spr Cmt \\
\hline HW-2 & 3.3 & 1.0 & 5.7 & 10.0 & 79.3 & 0.7 & 0.0 \\
\hline HW-5 & 0.0 & 0.3 & 18.3 & 14.3 & 67.0 & 0.0 & 0.0 \\
\hline LL-9 & 0.4 & 2.0 & 32.3 & 19.4 & 0.0 & 45.9 & 0.0 \\
\hline LL-10 & 8.4 & 1.0 & 4.0 & 20.0 & 46.3 & 20.3 & 0.0 \\
\hline SH-6a & 12.3 & 0.0 & 4.3 & 24.1 & 59.3 & 0.0 & 0.0 \\
\hline SH-6b & 19.0 & 0.4 & 11.7 & 8.0 & 53.9 & 7.0 & 0.0 \\
\hline ER-4c & 1.3 & 1.3 & 0.7 & 43.0 & 7.7 & 46.0 & 0.0 \\
\hline ER-5 & 0.0 & 0.1 & 2.0 & 22.0 & 75.9 & 0.0 & 0.0 \\
\hline ER-6 & 0.0 & 0.7 & 2.3 & 32.2 & 0.0 & 64.7 & 0.0 \\
\hline ER-7 & 0.7 & 0.7 & 0.0 & 36.4 & 0.0 & 62.3 & 0.0 \\
\hline ER-9a & 0.0 & 0.3 & 0.0 & 50.6 & 0.0 & 49.0 & 0.0 \\
\hline ER-9d & 1.3 & 0.3 & 1.4 & 27.4 & 61.3 & 8.3 & 0.0 \\
\hline DM-7 & 0.0 & 72.3 & 0.0 & 27.7 & 0.0 & 0.0 & 0.0 \\
\hline DM-8b & 0.0 & 0.0 & 100.0 & 0.0 & 0.0 & 0.0 & 0.0 \\
\hline DM-13 & 4.3 & 25.7 & 44.0 & 4.3 & 18.3 & 3.3 & 0.0 \\
\hline DM-15 & 12.7 & 8.0 & 13.0 & 34.0 & 21.3 & 11.0 & 0.0 \\
\hline DM-16 & 0.0 & 0.3 & 6.3 & 16.0 & 66.0 & 11.3 & 0.0 \\
\hline DM-17 & 5.3 & 0.4 & 3.7 & 37.4 & 34.0 & 19.3 & 0.0 \\
\hline DM-18 & 14.0 & 2.3 & 0.7 & 11.1 & 0.0 & 0.0 & 72.0 \\
\hline DM-19 & 0.0 & 14.4 & 0.0 & 29.0 & 26.3 & 30.3 & 0.0 \\
\hline
\end{tabular}


Table 6. This table shows the percent data for each of the categories and is arranged by the rock types.

\begin{tabular}{|c|c|c|c|c|c|c|c|c|c|c|c|c|c|c|c|c|c|c|c|c|c|c|c|c|c|c|c|c|}
\hline \multirow[b]{2}{*}{ 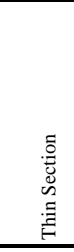 } & \multicolumn{4}{|c|}{$\begin{array}{c}\text { Non-Skeletal } \\
\text { Carbonate Grains }\end{array}$} & \multicolumn{5}{|c|}{ Diagenetic Minerals } & \multicolumn{3}{|c|}{ Silicicllastic Sediments } & \multicolumn{13}{|c|}{ Fossils } & \multicolumn{2}{|c|}{ Matrix } & \multirow[b]{2}{*}{ 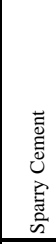 } \\
\hline & 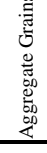 & 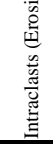 & 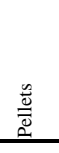 & 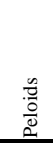 & $\begin{array}{l}\text { 言 } \\
\frac{\tilde{E}}{\tilde{E}} \\
\end{array}$ & 苞 & 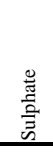 & 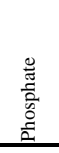 & 总 & 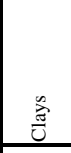 & 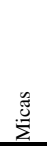 & $\begin{array}{l}\frac{0}{0} \\
\frac{\tilde{g}}{0} \\
\end{array}$ & 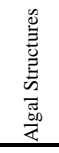 & 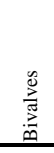 & 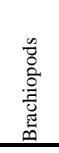 & 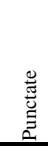 & 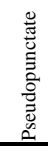 & 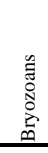 & 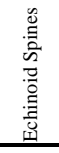 & $\begin{array}{l}\text { 量 } \\
\text { 总 } \\
\end{array}$ & 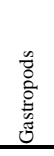 & $\begin{array}{l}\mathscr{\mathscr { \Xi }} \\
\stackrel{亏}{0} \\
\tilde{\Xi} \\
\end{array}$ & 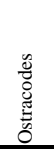 & 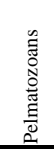 & 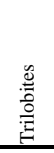 & 莺 & 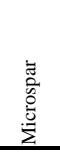 & \\
\hline \multicolumn{29}{|c|}{\begin{tabular}{|l} 
Wackestones \\
\end{tabular}} \\
\hline $\mathrm{HW}-2$ & 0.0 & 0.0 & 0.0 & 3.3 & 0.0 & 0.0 & 0.0 & 0.3 & 0.7 & 2.7 & 0.0 & 3.0 & 2.0 & 0.0 & 3.3 & 0.0 & 0.0 & 2.0 & 0.0 & 0.0 & 0.0 & 0.0 & 0.7 & 2.0 & 0.0 & 79.3 & 0.7 & 0.0 \\
\hline $\mathrm{HW}-5$ & 0.0 & 0.0 & 0.0 & 0.0 & 0.3 & 0.0 & 0.0 & 0.0 & 0.0 & 0.3 & 0.0 & 18.0 & 13.7 & 0.7 & 0.0 & 0.0 & 0.0 & 0.0 & 0.0 & 0.0 & 0.0 & 0.0 & 0.0 & 0.0 & 0.0 & 67.0 & 0.0 & 0.0 \\
\hline DM-15 & 0.0 & 0.0 & 0.0 & 12.7 & 0.0 & 7.3 & 0.0 & 0.0 & 0.7 & 11.3 & 0.0 & $\begin{array}{ll}1.7 \\
\end{array}$ & $\begin{array}{l}9.0 \\
\end{array}$ & 6.3 & $\begin{array}{ll}3.7 \\
\end{array}$ & 0.0 & 0.3 & 0.7 & $\begin{array}{l}0.0 \\
\end{array}$ & 1.3 & 0.7 & 0.3 & 1.0 & 10.7 & 0.0 & 21.3 & 11.0 & 0.0 \\
\hline DM-16 & 0.0 & 0.0 & 0.0 & 0.0 & 0.0 & 0.0 & 0.0 & 0.0 & 0.3 & 0.0 & 2.3 & 4.0 & 0.3 & 3.0 & 3.3 & 0.0 & 1.3 & 1.0 & 0.3 & 1.0 & 0.3 & 0.0 & 0.3 & 4.7 & 0.3 & 66.0 & $\begin{array}{ll}11.3 \\
\end{array}$ & 0.0 \\
\hline \multirow{2}{*}{\multicolumn{29}{|c|}{$\frac{1}{\text { Skeletal Wackestones }}$}} \\
\hline & & & & & & & & & & & & & & & 4.7 & 0.0 & 0.3 & 0.3 & 0.0 & 0.7 & 0.0 & 0.0 & 0.0 & 3.7 & 0.3 & 46.3 & 20.3 & 0.0 \\
\hline SH-6a & 0.0 & 5.3 & 0.0 & 7.0 & 0.0 & 0.0 & 0.0 & 0.0 & 0.0 & 3.7 & 0.3 & 0.3 & $\begin{array}{ll}0.7 \\
\end{array}$ & 5.3 & 5.0 & 0.0 & 0.0 & 0.3 & 0.0 & 5.0 & 0.0 & 0.0 & 0.7 & 7.0 & 0.0 & 59.3 & 0.0 & 0.0 \\
\hline ER-4c & 0.0 & 0.0 & 0.0 & 1.3 & 0.0 & 0.0 & 0.0 & 0.3 & 1.0 & 0.7 & 0.0 & 0.0 & 0.0 & 2.3 & 14.0 & 0.0 & 4.3 & 1.0 & 0.0 & 2.7 & 0.7 & 0.0 & 0.3 & 17.0 & 0.7 & 7.7 & 46.0 & 0.0 \\
\hline ER-5 & 0.0 & 0.0 & 0.0 & 0.0 & 0.0 & 0.0 & 0.0 & 0.0 & 0.0 & 0.0 & 0.0 & 2.0 & 0.0 & 2.0 & 6.0 & 0.0 & 2.3 & 0.0 & 0.0 & 6.7 & 0.0 & 0.0 & 0.7 & 4.3 & 0.0 & 75.9 & 0.0 & 0.0 \\
\hline ER-9d & 0.0 & 0.0 & 0.0 & 1.3 & 0.0 & 0.0 & 0.0 & 0.0 & 0.3 & 0.0 & 0.0 & 1.3 & $\begin{array}{ll}1.7 \\
\end{array}$ & 0.0 & 9.0 & 0.0 & 1.0 & 4.0 & 0.0 & 2.0 & 0.0 & 2.0 & 0.3 & 7.3 & 0.0 & 61.3 & 8.3 & 0.0 \\
\hline DM-19 & 0.0 & 0.0 & 0.0 & 0.0 & 3.0 & 0.0 & 11.3 & 0.0 & 0.0 & 0.0 & 0.0 & 0.0 & 0.0 & 4.0 & 11.7 & 0.0 & 0.3 & 2.3 & 0.0 & 2.0 & 4.7 & 0.0 & 1.0 & 3.0 & 0.0 & 26.3 & 30.3 & 0.0 \\
\hline \multirow{2}{*}{\multicolumn{29}{|c|}{ Packstones }} \\
\hline & 0.0 & 0.0 & 0.0 & 0.3 & 0.0 & 0.0 & 0.0 & 0.0 & 2.0 & 28.3 & 0.0 & 4.0 & 1.0 & 4.3 & 10.0 & 0.0 & 1.0 & 0.3 & 0.0 & 0.0 & 0.0 & 0.0 & 0.0 & 2.7 & 0.0 & 0.0 & 45.9 & 0.0 \\
\hline ER-9a & 0.0 & 0.0 & 0.0 & 0.0 & 0.0 & 0.0 & 0.0 & 0.0 & 0.3 & 0.0 & 0.0 & 0.0 & 0.0 & 0.3 & 44.0 & 0.0 & 1.7 & 1.3 & 0.0 & 0.0 & 0.0 & 0.0 & 0.0 & 3.3 & 0.0 & 0.0 & 49.0 & 0.0 \\
\hline \multicolumn{29}{|c|}{ Skeletal Packstones } \\
\hline ER-7 & 0.0 & 0.0 & 0.0 & 0.7 & 0.0 & 0.3 & 0.0 & 0.0 & 0.3 & 0.0 & 0.0 & 0.0 & 0.0 & 7.0 & 18.3 & 0.0 & 3.0 & 0.0 & 0.3 & 0.0 & 0.7 & 0.0 & 0.0 & 6.7 & 0.3 & 0.0 & 62.3 & 0.0 \\
\hline DM-17 & 0.0 & 0.0 & 0.0 & 5.3 & 0.0 & 0.0 & 0.0 & 0.0 & 0.3 & 2.0 & 0.3 & 1.3 & 0.3 & 0.7 & 2.7 & 0.0 & 1.0 & 1.7 & 1.0 & 1.3 & 0.0 & 0.0 & 0.7 & 27.6 & 0.3 & 34.0 & 19.3 & 0.0 \\
\hline \multicolumn{29}{|c|}{\begin{tabular}{|l} 
Peloidal Pkst / Mdst \\
\end{tabular}} \\
\hline SH-6b & 0.0 & 0.0 & 0.0 & 19.0 & 0.0 & 0.3 & 0.0 & 0.0 & 0.0 & 1.7 & 1.0 & 9.0 & 0.3 & 1.0 & 1.7 & 0.7 & 0.0 & 0.0 & 0.0 & 0.7 & 0.0 & 0.0 & 1.0 & 2.7 & 0.0 & 53.9 & 7.0 & 0.0 \\
\hline DM-18 & 0.0 & 0.0 & 0.0 & 14.0 & 0.0 & 0.0 & 0.0 & 0.0 & 2.3 & 0.0 & 0.0 & $\begin{array}{ll}0.7 \\
\end{array}$ & 0.0 & 0.0 & 2.0 & 0.0 & 1.0 & 0.0 & 0.0 & 7.0 & 0.0 & 0.0 & 1.0 & 0.0 & 0.0 & 0.0 & 0.0 & 72.0 \\
\hline \multicolumn{29}{|c|}{ Poorly Washed Gst. / Pkst. } \\
\hline ER-6 & 0.0 & 0.0 & 0.0 & 0.0 & 0.7 & 0.0 & 0.0 & 0.0 & 0.0 & 2.3 & 0.0 & 0.0 & $\begin{array}{ll}0.3 \\
\end{array}$ & 5.7 & 12.4 & 0.3 & 2.3 & 0.7 & 0.3 & 3.0 & 2.7 & 0.0 & 0.0 & 4.4 & 0.0 & 0.0 & 64.7 & 0.0 \\
\hline \multicolumn{29}{|c|}{\begin{tabular}{|l|l|}
\multicolumn{2}{|c|}{$\mid$} \\
Boundstones \\
DM-7 & 0.0 \\
\end{tabular}} \\
\hline DM-7 & 0.0 & 0.0 & 0.0 & 0.0 & 0.0 & 72.3 & 0.0 & 0.0 & 0.0 & 0.0 & 0.0 & 0.0 & 27.7 & 0.0 & 0.0 & 0.0 & 0.0 & 0.0 & 0.0 & 0.0 & 0.0 & 0.0 & 0.0 & 0.0 & 0.0 & 0.0 & 0.0 & 0.0 \\
\hline \multicolumn{29}{|c|}{\begin{tabular}{|l|} 
Calcareous Siltstones \\
\end{tabular}} \\
\hline DM-8b & 0.0 & 0.0 & 0.0 & 0.0 & 0.0 & 0.0 & 0.0 & 0.0 & 0.1 & 208.0 & 3.0 & 89.0 & 0.0 & 0.0 & 0.0 & 0.0 & 0.0 & 0.0 & 0.0 & 0.0 & 0.0 & 0.0 & 0.0 & 0.0 & 0.0 & 0.0 & 0.0 & 0.0 \\
\hline DM-13 & 0.0 & 0.0 & 0.0 & 4.3 & 0.0 & 0.0 & 0.0 & 18.3 & 7.3 & 0.0 & 2.0 & 42.0 & 0.0 & 2.0 & 1.7 & 0.0 & 0.0 & 0.0 & 0.0 & 0.0 & 0.0 & 0.0 & 0.0 & 0.3 & 0.3 & 18.3 & 3.3 & 0.0 \\
\hline
\end{tabular}


Table 7. These are point count percent category totals condensed from Table 6. Averages have been reported where multiple units are characterized by a single lithology.

\begin{tabular}{|c|c|c|c|c|c|c|c|}
\hline & Non-Skel & Diagenetic & Siliciclastic & Fossil & Micrite & Mic Spr & Spr Cmt \\
\hline \multicolumn{8}{|c|}{$\frac{1}{\text { Wackestones }}$} \\
\hline HW-2 & 3.3 & 3.0 & 3.7 & 10.0 & 79.3 & 0.7 & 0.0 \\
\hline HW-5 & 0.0 & 0.7 & 18.0 & 14.3 & 67.0 & 0.0 & 0.0 \\
\hline DM-15 & 12.7 & 8.0 & 13.0 & 34.0 & 21.3 & 11.0 & 0.0 \\
\hline DM-16 & 0.0 & 0.3 & 6.3 & 16.0 & 66.0 & 11.3 & 0.0 \\
\hline$\overline{\mathrm{Avg}}$ & 4.0 & 3.0 & 10.2 & 18.6 & 58.4 & 5.7 & 0.0 \\
\hline \multicolumn{8}{|c|}{\begin{tabular}{|l} 
Skeletal Wackestones \\
\end{tabular}} \\
\hline LL-10 & 8.4 & 1.0 & 4.0 & 20.0 & 46.3 & 20.3 & 0.0 \\
\hline SH-6a & 12.3 & 0.0 & 4.3 & 24.1 & 59.3 & 0.0 & 0.0 \\
\hline ER-4c & 1.3 & 1.3 & 0.7 & 43.0 & 7.7 & 46.0 & 0.0 \\
\hline ER-5 & 0.0 & 0.1 & 2.0 & 22.0 & 75.9 & $\frac{40.0}{0.0}$ & 0.0 \\
\hline ER-9d & 1.3 & 0.3 & 1.4 & 27.4 & 61.3 & 8.3 & 0.0 \\
\hline DM-19 & 0.0 & 14.4 & 0.0 & 29.0 & 26.3 & 30.3 & 0.0 \\
\hline Avg & 3.9 & 29 & 2.1 & 27.6 & 46.1 & 17.5 & 0.0 \\
\hline \multicolumn{8}{|c|}{$\frac{1}{\text { Packstones }}$} \\
\hline LL-9 & 0.4 & 2.0 & 32.3 & 19.4 & 0.0 & 45.9 & 0.0 \\
\hline ER-9a & 0.0 & 0.3 & 0.0 & 50.6 & 0.0 & 49.0 & 0.0 \\
\hline Avg & 0.2 & 1.2 & 16.2 & 35.0 & 0.0 & 47.4 & 0.0 \\
\hline \multicolumn{8}{|c|}{$\begin{array}{c}\text { Skeletal Packstones } \\
\end{array}$} \\
\hline ER-7 & 0.7 & 0.7 & 0.0 & 36.4 & 0.0 & 62.3 & 0.0 \\
\hline DM-17 & 5.3 & 0.4 & 3.7 & 37.4 & 34.0 & 19.3 & 0.0 \\
\hline Avg & 3.0 & 0.5 & 1.8 & 36.9 & 17.0 & 40.8 & 0.0 \\
\hline \multicolumn{8}{|c|}{ Peloidal Pkst / Mdst } \\
\hline SH-6b & 19.0 & 0.4 & 11.7 & 8.0 & 53.9 & 7.0 & 0.0 \\
\hline DM-18 & 14.0 & 2.3 & 0.7 & 11.1 & 0.0 & 0.0 & 72.0 \\
\hline Avg & 16.5 & 1.4 & 6.2 & 9.5 & 27.0 & 3.5 & 36.0 \\
\hline \multicolumn{8}{|c|}{ Poorly Washed Gst / Pkst } \\
\hline ER-6 & 0.0 & 0.7 & 2.3 & 32.3 & 0.0 & 32.2 & 0.0 \\
\hline \multicolumn{8}{|c|}{\begin{tabular}{|c|} 
Boundstones \\
\end{tabular}} \\
\hline \begin{tabular}{|l|} 
DM-7 \\
\end{tabular} & 0.0 & 72.3 & 0.0 & 27.7 & 0.0 & 0.0 & 0.0 \\
\hline \multicolumn{8}{|c|}{\begin{tabular}{|l} 
Calcareous Siltstones \\
\end{tabular}} \\
\hline DM-8b & 0.0 & 0.0 & 100.0 & 0.0 & 0.0 & 0.0 & 0.0 \\
\hline DM-13 & 4.3 & 25.7 & 44.0 & 4.3 & 18.3 & 3.3 & 0.0 \\
\hline Avg & 2.2 & 12.9 & 72.0 & 2.2 & 9.2 & 1.7 & 0.0 \\
\hline
\end{tabular}




\section{Lower Clastic Unit}

Clay/Claystone

SH-2, 4.

Rocks in this category display a fine-grained, cohesive matrix with no apparent bedding structures. Grain size is dominantly clay with some interspersed silt and fine sand. Colors vary from grey to red but generally red/brown. These units are slightly calcareous at irregular intervals. These units are devoid of fossil material.

No sedimentary structures were identified in these units.

Fossiliferous Shales CH-3a, b; SH-5; EK-2, 7, 8.

Colors for these units range from green/grey to red/brown. Fossil content within the units varies from poorly to mildly fossiliferous. Fossils found in these units include: brachiopods, bivalves, burrowing bivalves, pectens, gastropods, and pelmatozoan debris. Other constituents within these shales vary. Some are relatively pure clay, with others having abundant micaceous and organic material.

A few sedimentary structures were observed in these fissile rocks with soft sediment deformational features the most common.

Non-fossiliferous Shales EK-12, 13; ER-2, 13; DM-2.

Colors for these units range from grey to red/brown and tan. All of these units are barren of fossil material. Grain constituents within these shales vary as well. Some are relatively pure clay, with others having abundant micaceous and organic material. 
Sedimentary structures observed were soft sediment deformation and rare Rusophycus traces.

Fossiliferous Mudstones

LL-5, 8a, b; EK-6.

Mudstones are characterized by their "blocky" weathering pattern. Colors range from grey to red. Oxidized colors (red/brown) were much more common. Fossil content within these units varies from barren to sparsely fossiliferous. Fossils found include: ostracodes, brachiopods - Lingula, and bivalves.

No sedimentary structures were noted in these units.

Non-fossiliferous Mudstones

HW-1; LL-3, 6; SH-3; EK-4; DM-3, 5, 9, 11, 12.

Colors range from green/grey to red and some are mottled. Mottled colors (red/green) were more common in these units. All these units were barren of fossil material. No sedimentary structures were noted in these units.

Siltstone LL-2, 4; CH-2a, b, c, 4.

The siltstone lithofacies is identified by its fine grained and diffuse bedding. These rocks are much more resistant to weathering than shale or mudstone and are much more prominent in outcrop. Colors range from green/tan to red and some units show green/red mottling. All units are barren of fossil material.

The only sedimentary structure seen in the field was bioturbation by plant roots. 


\section{Fossiliferous Sandstones}

EK-7; ER-2d; DM-6.

This sandstone lithofacies ranges from very fine- to medium-grained quartz sand. Colors for these units vary from tan to grey. Fossil content ranges from poorly to mildly fossiliferous. Horizontal trace fossils were found at the tops of some beds and bioturbation was noted sporadically throughout. Fossil fragments and organic debris were noted infrequently. Overall, units in the sandstone lithofacies are dominantly silica-cemented quartz sands, but a few of the units are slightly calcareous. Rock fragments and micas are present in some units.

Sedimentary structures commonly found in the sandstones; small-scale cross-beds, ripples, and trough cross-beds. Some units have small-scale erosional bases with scour-and-fill structures.

Non-fossiliferous Sandstones EK-3, 5; DM-4, 10a, b, c, d, e, f, g.

Sandstones in this lithofacies range from very fine- to medium-grained quartz sand. Colors for these units vary from green/tan to red/brown. Overall, units in the sandstone lithofacies are dominantly silica-cemented quartz sands, but a few of the units are slightly calcareous. Chert nodules are present but rare. Rock fragments and micas are present in some units. These sands are devoid of body fossil material although some terrestrial plant fragments found sporadically.

Sedimentary structures are found commonly in the sandstones. Small-scale cross-beds, ripples, and channel forms are common. Some units have small-scale erosional bases with scour-and-fill structures. 
Non-fossiliferous Limestones

ER-2a, b, c.

Colors within these limestones vary from grey on freshly broken surfaces to tan on weathered surfaces. These are dominantly ooid grainstones with occasional mudstones. All units are barren of fossil material.

No sedimentary structures were noted.

\section{Fossiliferous Limestones}

LL-7.

This unit is medium grey where fresh and weathers to a tan/brown. It is a thinly bedded argillaceous wackestone. Fossils are found throughout and are abundant. Both the lateral thickness and amount of argillaceous material vary laterally within this unit.

No sedimentary structures were observed for this unit.

\section{Calcareous Siltsone}

DM-8a, b*.

This unit is yellow/tan on both freshly broken and weathered surfaces. This unit is barren of fossil material. The overall grain size for this unit is medium to fine silt.

No sedimentary structures were noted for this unit at outcrop scale, however, micrograded beds were found at outcrop scale (Plate 8a, b). 


\section{Plate 8}
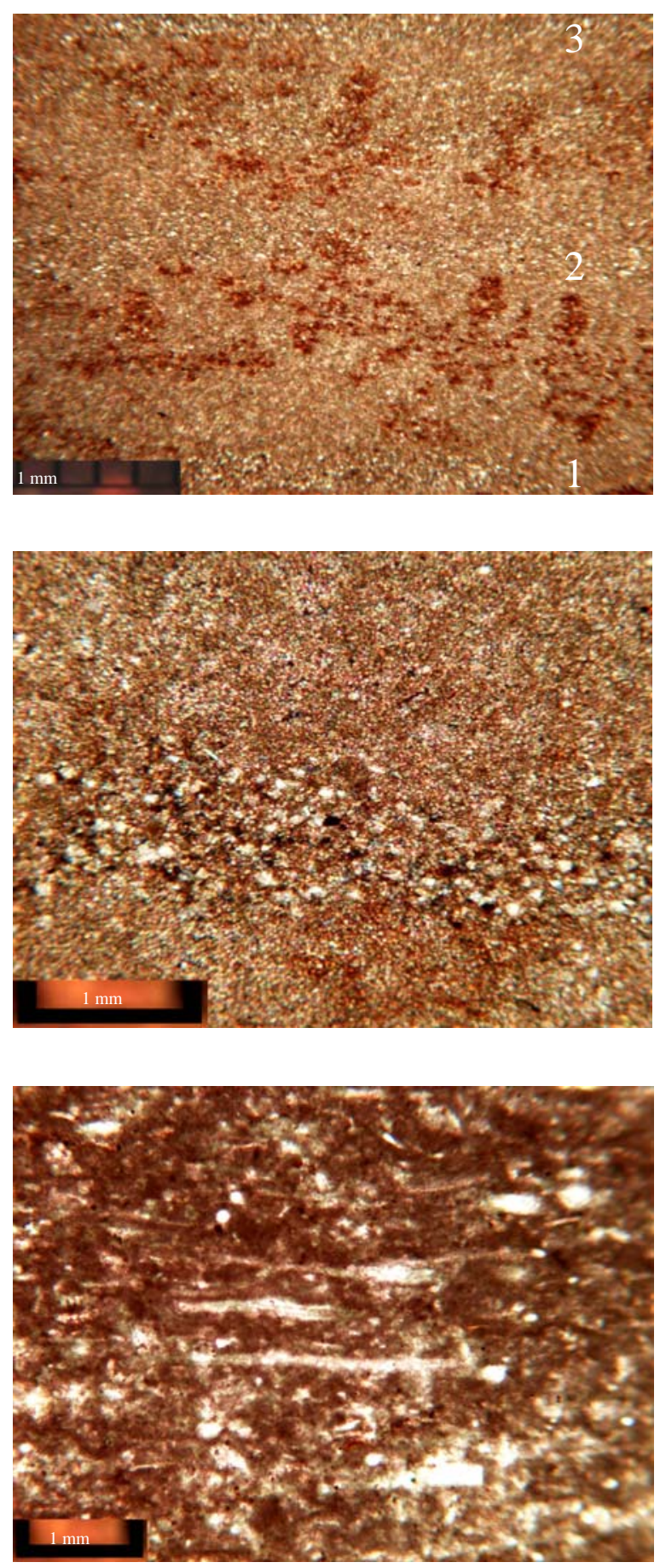

8a. Calcareous Siltstone at 2.5x; DM-8b. Three micro-graded bed sequences are visible in this photo. Note the dendritic iron staining pattern.

8b. Calcareous Siltstone at 10x; DM-8b. This is an enlargement of the lowermost micro-graded bed from photo 8 a.

8c. Microbial Boundstone at 10x; DM-7. The important features in this slide are the elongate lighter colored laminae inside the overall dark background. 
Boundstones

DM-7*. (Plate 8c)

These rocks are described as a ripple laminated sparsely fossiliferous mudstone in outcrop. Upon thin-section identification, it became apparent that the ripple laminae were actually remnant traces of wavy microbial mats. This unit is medium grey on freshly broken surfaces and weathers to a tan / brown color.

This unit is composed of two major grain types: diagenetic and fossil grains. All other grain types amount to $0.0 \%$ for this unit. The diagenetic grains, exclusively chert, account for the dominant percent of this rock at 72.3\%. Fossil grains, composed exclusively of skeletal microbial structures, account for the remaining $27.7 \%$ of the rock.

No early diagenetic minerals or sedimentary structures were noted for these rocks.

\section{Middle Carbonate Unit}

Wackestones

HW-2*, 5*, DM-15*, 16*. (Plate 9a)

Field descriptions for wackestones often identified these rocks as mudstones because of the overall fine grain size of the samples. All fossil material was reported as unidentifiable fossil bits/hash in the field descriptions. These rocks are light grey on freshly broken surfaces and weather to a yellow-tan.

Micrite is by far the most dominant matrix type accounting for $58.4 \%$ of the total. Fossil grains are $18.6 \%$ of the total with the dominant grain types: calcareous algal encrusters, brachiopods and pelmatozoan debris. Siliciclastic sediments account for $10.2 \%$ of the total with quartz as the dominant siliciclastic sediment but clays and micas are also present. 


\section{Plate 9}

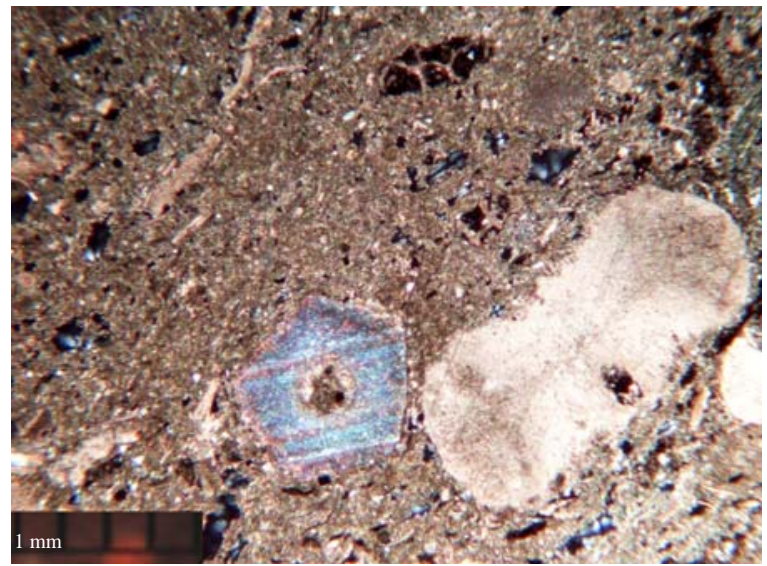

9a. Wackestone at 2.5x; HW-2. This slide illustrates the carbonate matrix as well as some fossil grains: top center - brown grain - bryozoan; bottom center - blue grain - pelmatozoan debris.

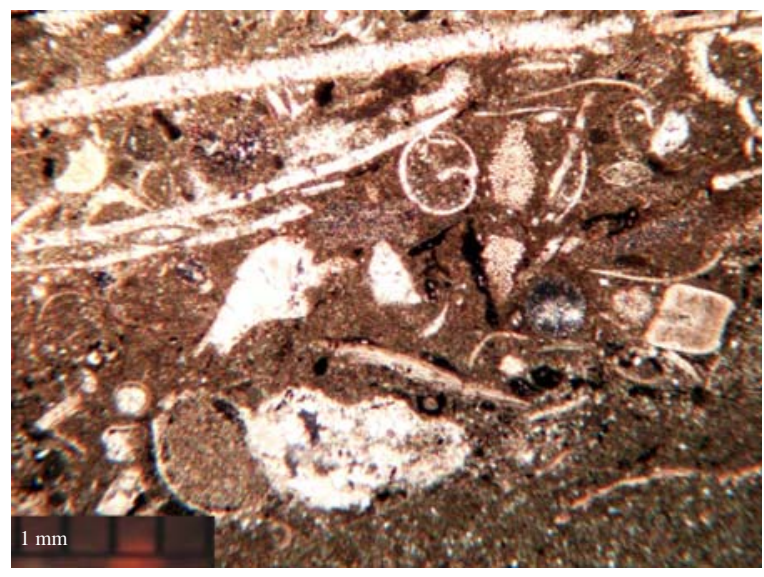

9b. Skeletal Wackestone at 2.5x; DM-15. A variety of skeletal grains can be seen in this slide: top left - brachiopod material; middle right - pelmatozoan debris; bottom center gastropod.

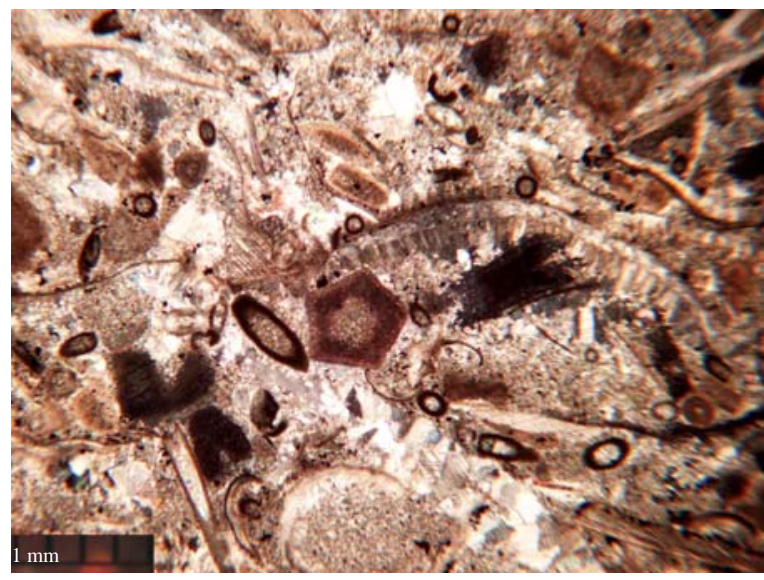

9c. Skeletal Packstone at 2.5x; ER-9a. Numerous skeletal fragments are visible including: right center - brachiopod material; middle center pelmatozoan debris. 
and non-carbonate grains are very minor grain constituents in the overall lithology accounting for a mere $1.1 \%$ and $1.3 \%$ respectively. Pyrite is the most common non-carbonate diagenetic grain in these samples, but chalcedony is also present in minor amounts.

The diagenetic minerals noted in the thin sections were pyrite and chalcedony.

Sedimentary structures observed at the outcrop include laminae and small-scale ripples.

\section{Skeletal Wackestones}

LL-10*, SH-6a*, ER-4c*, 5*, 9d*; DM-19*. (Plate 9b)

These rocks were described as fossiliferous wackestones in the field. The most notable characteristic for all of these rocks is the abundant fossil material. These rocks display a medium grey color on freshly broken surfaces and weather to a tan / yellow-tan color.

Micrite is the dominant matrix type at $46.1 \%$. Fossil grains account for a large percent of the overall total at $27.6 \%$. Pelmatozoan debris, brachiopods, bivalves and echinoid spines are the most significant fossil grain types. Microspar is a significant percent of the overall rock character at $17.5 \%$. Non-skeletal carbonate grains comprise $3.9 \%$ of the overall rock with peloids and intraclasts as the dominant non-skeletal grain types. Diagenetic minerals account for a relatively small part of the overall rock make-up at 2.9\% with dominant grains as sulfates, chalcedony, and pyrite. Siliciclastic sediments, specifically quartz and clay, are a small part of these skeletal wackestones composing $2.1 \%$ of the total grain count.

The diagenetic minerals noted in the thin sections were pyrite, sulfates, and chalcedony. Sedimentary structures observed at the outcrops were laminae, small-scale ripples and crossbeds. 
$\underline{\text { Packstones }}$

LL-9*, ER-9a*. (Plate 9c, 10a)

These rocks were described as highly fossiliferous, argillaceous limestones and

fossiliferous packstones in the field. Fresh surfaces are generally grey; weathered surfaces are tan. The dominant matrix type for these packstones is micrite at 30.6\% with microspar at $27.1 \%$. Fossil grains are the dominant grain type and account for $23.4 \%$ of the total. Brachiopods are by far the dominant fossil grain with pelmatozoan debris and bivalves adding to the grain constituents. Siliciclastic sediments are a significant component of the overall lithology totaling 16.8\%. In these samples, clays are by far the dominant siliciclastic sediment with minor amounts of quartz. Non-carbonate diagenetic grains, exclusively pyrite, compose a small portion of the overall grains at $1.2 \%$. Non-skeletal carbonate grains (peloids) are an insignificant part of the rock make-up at $0.8 \%$.

The diagenetic mineral noted in the thin sections was pyrite.

No sedimentary structures were noted in the field for these units.

\section{Skeletal Packstones}

ER-7*, DM-17*.

These rocks were described as argillaceous fossiliferous wackestones in the field. Fresh surfaces are described as a grey brown, while weathered surfaces change to a brown/tan color. The dominant matrix type for these units are microspar at $41.0 \%$ and micrite at $17.0 \%$. Fossil grains account for $36.9 \%$ of the overall rock total with brachiopods, bivalves, and pelmatozoan debris comprising the majority of the total. Siliciclastic sediments contribute a minor amount to the overall rock total at 3.6\% with quartz grains and clays as the dominant of these sediments. 


\section{Plate 10}
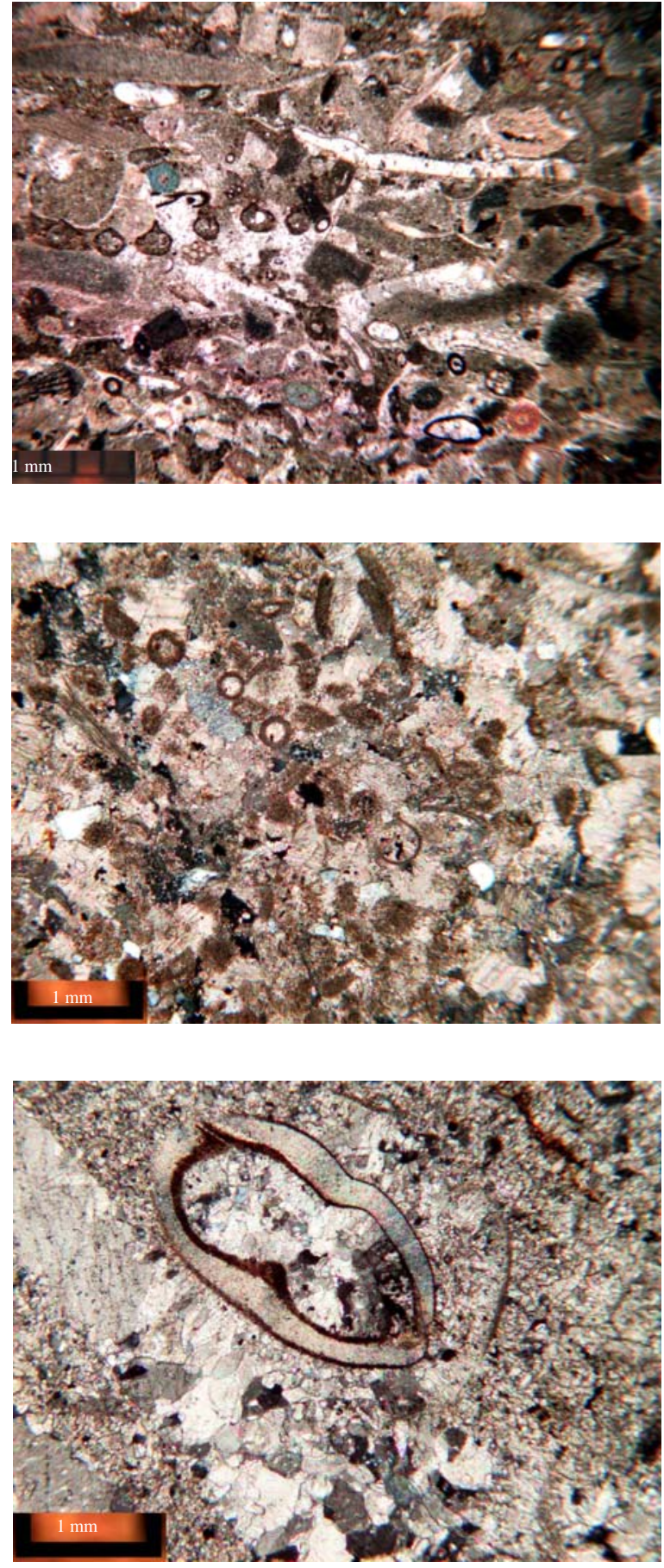

10a. Skeletal Packstone at 2.5x; LL-9. An abundance of fossil material: top center pelmatozoan debris; middle right - pseudopunctate brachiopod; middle left - bryozoan.

10b. Peloidal Packstone at 10x; DM-18. Peloidal material is represented by the brown ovals. 10c. Packstone / Grainstone at 10x; ER-6. This shows an ostracode, enclosed in a brown micrite envelope, directly adjacent to a brachiopod fragment. 
Diagenetic minerals are an insignificant part of the overall rock make-up and comprise $0.5 \%$ of the overall total.

The diagenetic minerals noted were chert and pyrite.

No sedimentary structures were noted for these units.

\section{Peloidal Mudstones / Packstones}

SH-6b*, DM-18*. (Plate 10b)

These rocks were described as argillaceous mudstone to mudstone in the field. On fresh surfaces, they display a light grey color and weather to tan. The dominant matrix types for these units are sparry cement at 36.0\% (packstone) and micrite at 27.0\% (mudstone). Non-skeletal carbonate grains are composed dominantly of peloids in this sample. These rocks contain the highest percent of peloids (16.5\%) of all thin section units. Siliciclastic sediments for this unit (6.2\%) are dominantly quartz with small amounts of clays and micas. Fossil grains, totaling $9.5 \%$ of the entire sample, are a minor constituent. Pelmatozoan debris is the highest-ranking fossil grain type followed by brachiopod and bivalve material. Microspar accounts for a minor part of the matrix at 3.5\%. Chert and pyrite, the only diagenetic non-carbonate grains, play an insignificant role accounting for only $1.3 \%$ of the total.

Pyrite and chert were the only diagenetic mineras noted in these thin sections.

No sedimentary structures were noted in the field.

\section{Poorly Washed Grainstones / Packstones}

ER-6*. (Plate 10c)

The grain-to-grain relationships and amount of microspar present place these rocks on the line between packstones and grainstones. However, these rocks are notably 
different from the other packstones previously described, as the amount of microspar in these samples is much higher. These units are light grey on freshly broken surfaces and weather to a yellow-tan.

The most significant matrix type in these samples is the microspar at $65.16 \%$. Fossil grains compose $32.25 \%$ of the overall total. The dominant fossil grains are brachiopods, pelmatozoan debris, forams and bivalves. Siliciclastic sediments total $2.36 \%$ of the overall rock make-up with clays comprising the dominant of these grains. Diagenetic and non-skeletal grains are very minor percentages of the total at $0.7 \%$ and $0.3 \%$ respectively with peloids being the only non-skeletal grain constituent. The diagenetic grains consist of trace amounts of chalcedony, chert, and pyrite. Siliciclastic sediments are dominantly clays with small amounts of quartz.

These grainstones/packstones have one of the lowest average siliciclastic sediment percentage of all the samples.

The diagenetic minerals noted in the thin sections were pyrite, chalcedony, and chert.

Sedimentary structures noted in the field for these units are channel fills, crossbeds, ripples, and bioturbation.

\section{Calcareous Siltstone}

DM-13*.

These rocks were described as silty/shaly mudstones in the field. Vertical and horizontal trace fossils and horizontal laminae were noted. These units are grey on freshly broken surfaces and weather to a grey/tan color.

Siliciclastic sediments, dominantly clay and quartz, make-up the bulk of this sample at 44.0\%. Phosphate and pyrite are the two major diagenetic grains making up the entirety of the $25.7 \%$ non-carbonate grain types. Micrite at $18.3 \%$ accounts for a notable percent of the overall 
rock total. A minor constituent of the grain total, peloids, account for only $2.2 \%$ of the nonskeletal carbonate grain total. Fossil grains within these samples are the lowest of any units sampled at 4.3\%. The dominant fossil grains are brachiopods and bivalves. The matrix totals for these rocks are also the lowest of all those studied comprising $21.6 \%$ of the total.

The diagenetic minerals noted in the thin sections were pyrite and phosphate.

Microlaminae, micro-graded beds, and burrows were observed in thin sections.

\section{Saprolitic Limestone}

CH-5.

These rocks have a claystone texture, but it is not described as such because numerous fossils are present. The fauna is diverse: brachiopods - Anthracospirifer, Composita, Orbiculoides, and Orthotetes; bivalves - burrowing clams, and pelmatozoan debris. This unit shows characteristics of being a leached limestone. Multiple horizons of color variation can be seen. The color variation from the top to bottom of the unit represents varied rates or exposure times of leaching by groundwater. The stratigraphic position of this unit above the Greenbrier and its thickness suggests that it is the erosional remnant of the Reynolds Limestone at Charcoal Hill.

Fossiliferous Mudstones / Shales HW-3; ER-6, 8, 9b, 9e; DM-14.

Colors for these units range from green/grey to red/brown. Fossil content within the units varies from poorly to mildly fossiliferous. Fossils found in these units include: brachiopods, bivalves, and ostracodes.

No sedimentary structures were observed. 
Non-fossiliferous Shales

EK-10; ER-4a, b.

Colors for these units range from grey to dark grey. All of these units are barren of fossil material. Interbedding between limestones and shales for these units is common.

No sedimentary structures were observed.

Upper Clastic Unit

HW-4, 6; LL-upper covered interval; CH-4, 6; SH-7, 8; EK-12, 13; ER-10, 11, 12, 13; DMupper covered interval.

Sandstones and shales of the same description as the Lower Clastic Unit.

\section{LITHOFACIES ASSOCIATIONS AND INTERPRETATIONS OF DEPOSITIONAL ENVIRONMENTS}

Environmental interpretations for this study will be concentrated around the Reynolds Limestone Member. However, to completely understand the paleoenvironmental conditions associated with the Reynolds, interpretations of the surrounding strata must also be made.

Mixed Carbonate and Siliciclastic Association

HW-1; LL-2, 3, 4, 5, 6, 7, 8a, b; CH-2a, b, c, 3a, b, 4; SH-2, 3, 4, 5; EK-2, 3, 4, 5, 6, 7, 8; ER-2a, b, c, d; DM-2, 3, 4, 5, 6, 7*, 8a, b*, 9, 10a, b, c, d, e, f, g, 11, 12

This lithofacies association contains a mixture of siliciclastic and carbonate deposits. The mixed carbonate and siliciclastic association occurs in strata lying between the top of the Greenbrier Limestone and the base of the Reynolds Limestone (Plate 11). The various 
lithologies within the lower clastic unit can be grouped by similar characteristics into different facies within the mixed carbonate and siliciclastic association.

Units that are dominantly non-fossiliferous and red/brown (oxidized) in color are associated with the various parts of a fluvial system. These units: clay/claystones, siltstones, and non-fossiliferous shales, mudstones (coastal plain); and sandstones (fluvial channels) exhibit characteristics typically identified in a fluvial system.

The lack of fossil material and oxidized color in these units suggests a terrestrial origin for these rocks. The absence of laterally continuous sedimentary structures suggests bioturbation by plant roots and possibly other organisms. All shale, mudstone, and claystone units are generally red in color and lack fossil material. Calcareous nodules are found in many of the mudstones. Mottling, suggesting pedogenesis, and the presence of calcareous nodules strongly indicate soil development. The sandstone is typically medium- to fine-grained and exhibits a scoured base, channel form, small-scale crossbeds and ripples; terrestrial plants and organic debris are also commonly found. All of these units exhibit characteristics associated with a fluvial system (Cant, 1982).

The calcareous siltstone is interpreted as a tidal flat environment (supratidal). The total absence of any fossil material and the presence of micro-graded beds from thin section identification support this interpretation (Laporte, 1967; Shinn, 1983). The silt-sized grains would have been easily disturbed by bioturbation action from either burrowing organisms or plant roots. The preservation of such finely graded beds suggests the environment was too harsh to support biota capable of destroying these graded beds.

The microbial boundstone is also interpreted to be a part of the tidal flat environment (intertidal). The absence of any fossil material other than the microbial mats and the 
preservation of their internal structure suggest environmental conditions here were also too harsh to support biota capable of disturbing them. Ripple laminations preserved at outcrop scale also support the intertidal interpretation (Laporte, 1967; Shinn, 1983).

The fossiliferous sandstones are interpreted to be a near-shore marine environment. These sands exhibit characteristics of in-shore sands with their sedimentary structures; smallscale ripples, cross beds, and trough cross beds and erosive bases (Inden and Moore, 1983). The presence of rock fragments and micas indicate fluvial influence and further support their nearshore interpretation. Fossils found in these units include: brachiopods - Anthracospirifer, Diaphragmus, Orthotetes, bivalves - Wilkingia, pelmatozoan debris, unidentifiable fossil hash, bioturbation, and burrows. This faunal assemblage is dominated by normal marine taxa and indicates deposition under normal marine conditions (Kammer and Lake, 2001).

The fossiliferous shales are interpreted to be a nearshore environment. Colors within these units (oxidized) and the presence of abundant micaceous and organic material suggest these units were nearshore. The red coloration of these shales does not necessarily result from subaerial exposure. This coloration can be explained through the transportation and deposition of red sediments into a carbonate setting - also supporting the nearshore interpretation. The diverse taxonomic assemblage: brachiopods - Anthracospirifer, Diaphragmus, Orbiculoides, Orthotetes; bivalves - Aviculopectin, Edmondia, Wilkingia, burrowing bivalves, pectens; gastropods, and pelmatozoan debris suggest deposition under normal marine conditions (McKerrow, 1978; Kammer and Lake, 2001).

The fossiliferous mudstones are interpreted to represent deposition in a stressed nearshore environment. Color variation in these units ranges from red to gray with some mottling. Fossils found within these units: brachiopods - Anthracospirifer, Orthotetes, Lingula; bivalves, 
Ostracodes, and gastropods indicate restricted marine or brackish water conditions (McKerrow, 1978; Kammer and Lake, 2001).

The non-fossiliferous limestone units are dominantly ooid grainstones. The lack of fossil material suggests environmental conditions were not suited to support biologic activity. Their position in relation to the surrounding strata suggests a brief period of deeper water $(\leq 2 \mathrm{~m})$ in a relatively shallow water setting. These ooid grainstones likely record the movement of an ooid bar or sand shoal across section in the lower clastic unit (Halley et al, 1983; Boardman et al, 1993).

The fossiliferous limestones in this unit have a composition and fossil assemblage suggesting deposition in shallow normal marine conditions (Kammer and Lake, 2001). Fossils found in this unit are brachiopods - Anthracospirifer, Composita, Diaphragmus, Eumetria, Orthotetes; others - pelmatozoan debris, an orthocones, bryozoans, and microbial mats (thin section). Sedimentary structures (sandy laminations) also indicate deposition in a shallow marine environment.

The vertical relationship of these units and their unique mixture in section suggests an interfingering of fluvial/terrestrial and carbonate environments typified by a marginal marine setting (Fig. 7a) (Boggs, 1987).

\section{Calcareous Siltstone Association}

DM-13*.

The dominant lithology in this association is calcareous siltstone. Clastic influence in this facies is among the highest of all those in the study area (Table 8). This unit shows intact 


\section{Models for Environmental Interpretations}

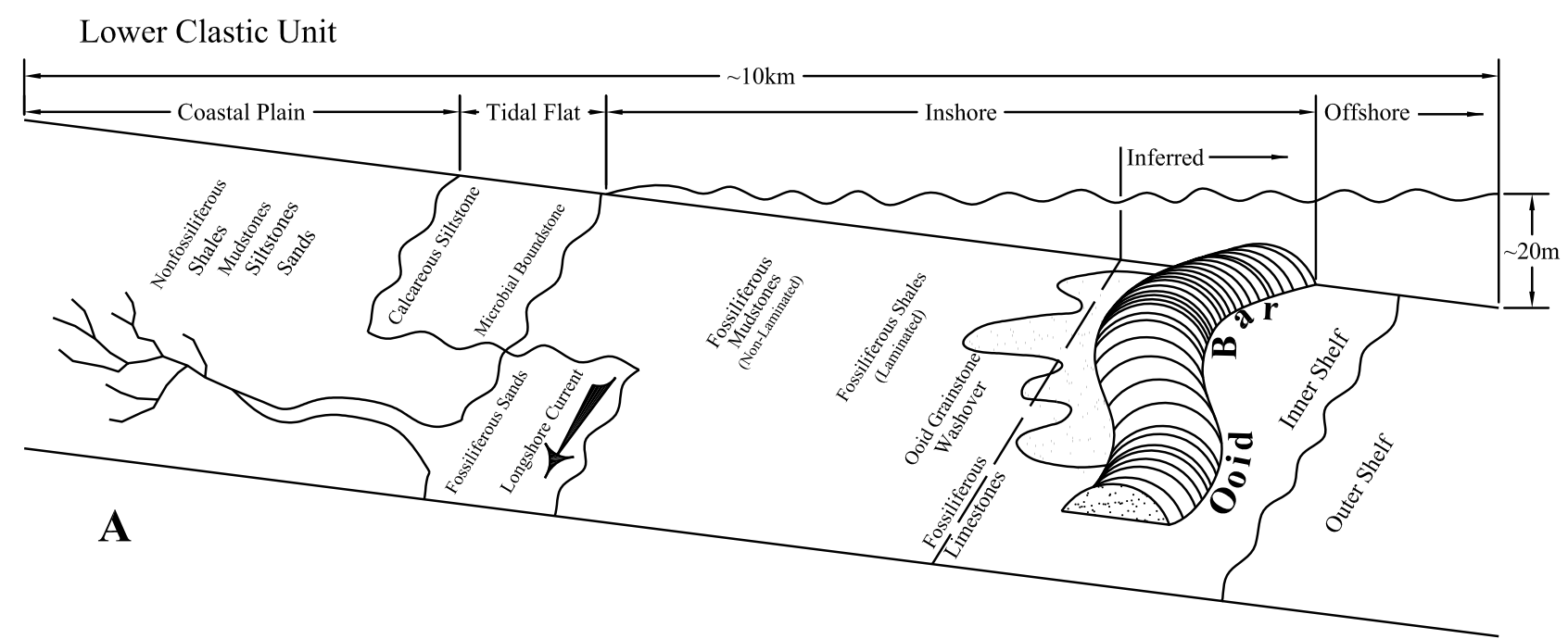

Middle Carbonate Unit

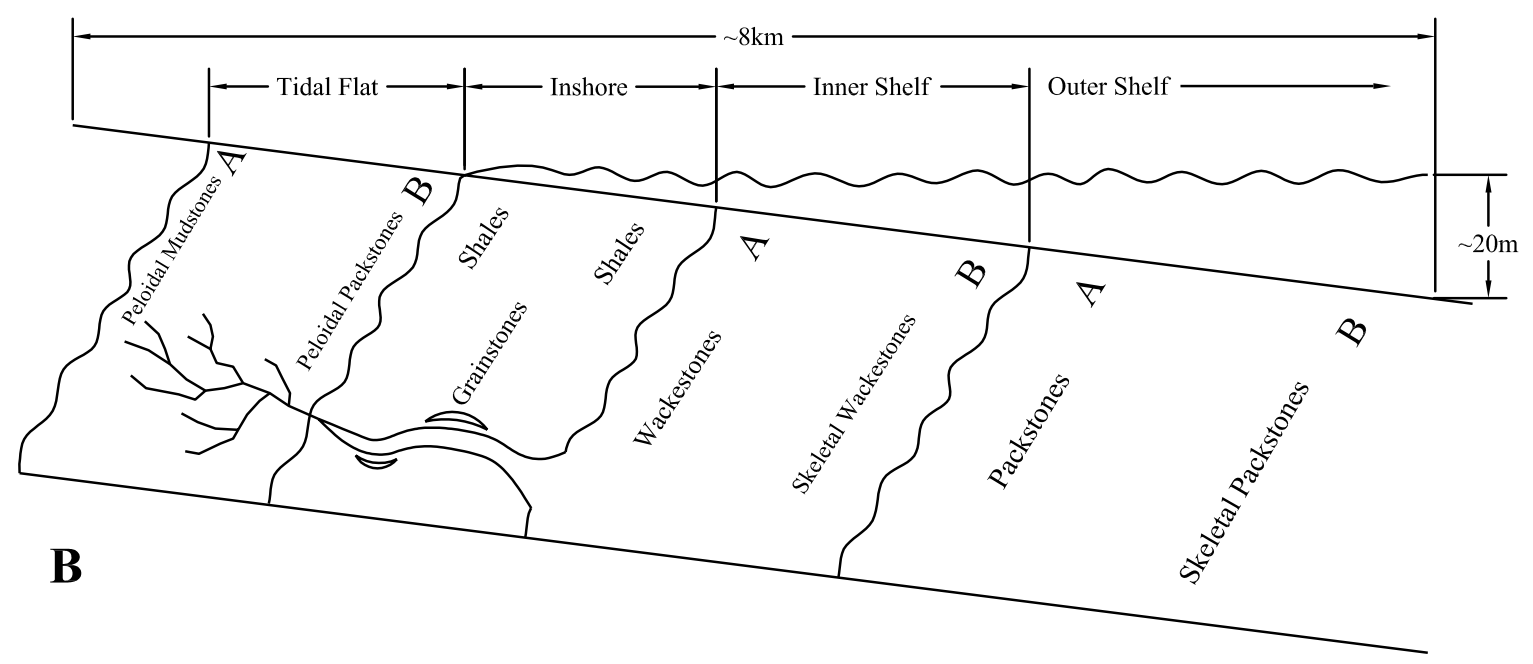

Figure 7. Depositional models illustrating the facies relationships for the environmental interpretations. 7A is a schematic representation of the three dimensional relationships of the facies for the Lower Clastic Unit. Notice how the environments grade into another. Position of the ooid bar is inferred as it is not found within the study area. 7B shows the facies relationships of the Middle Carbonate Unit during Reynolds Limestone deposition. The significant difference is the absence of significant siliciclastic influx. 


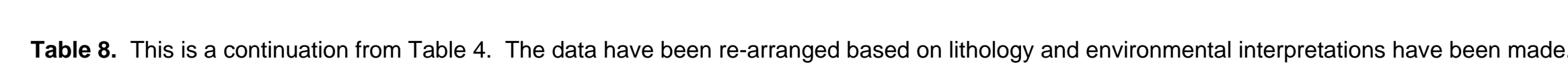

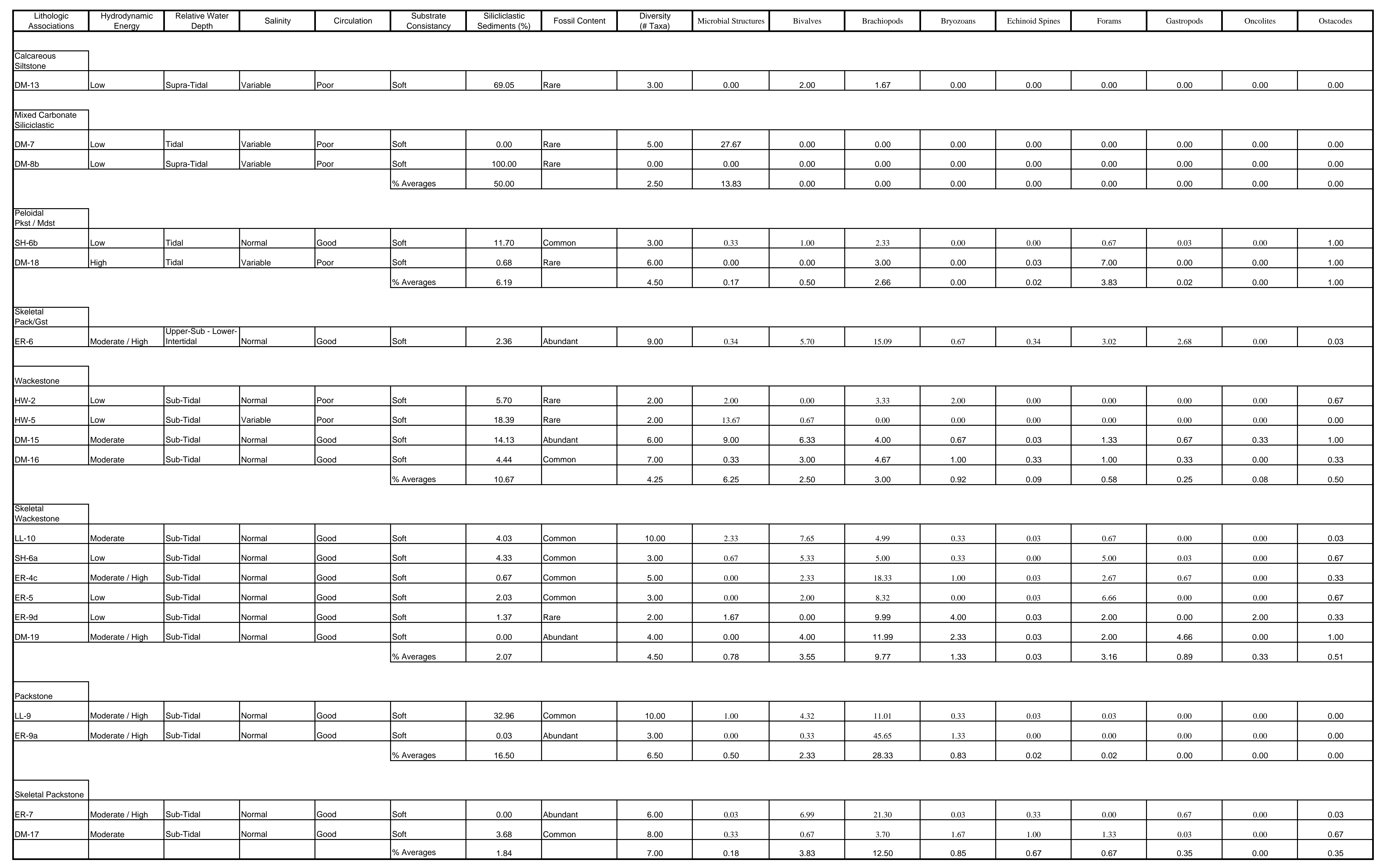




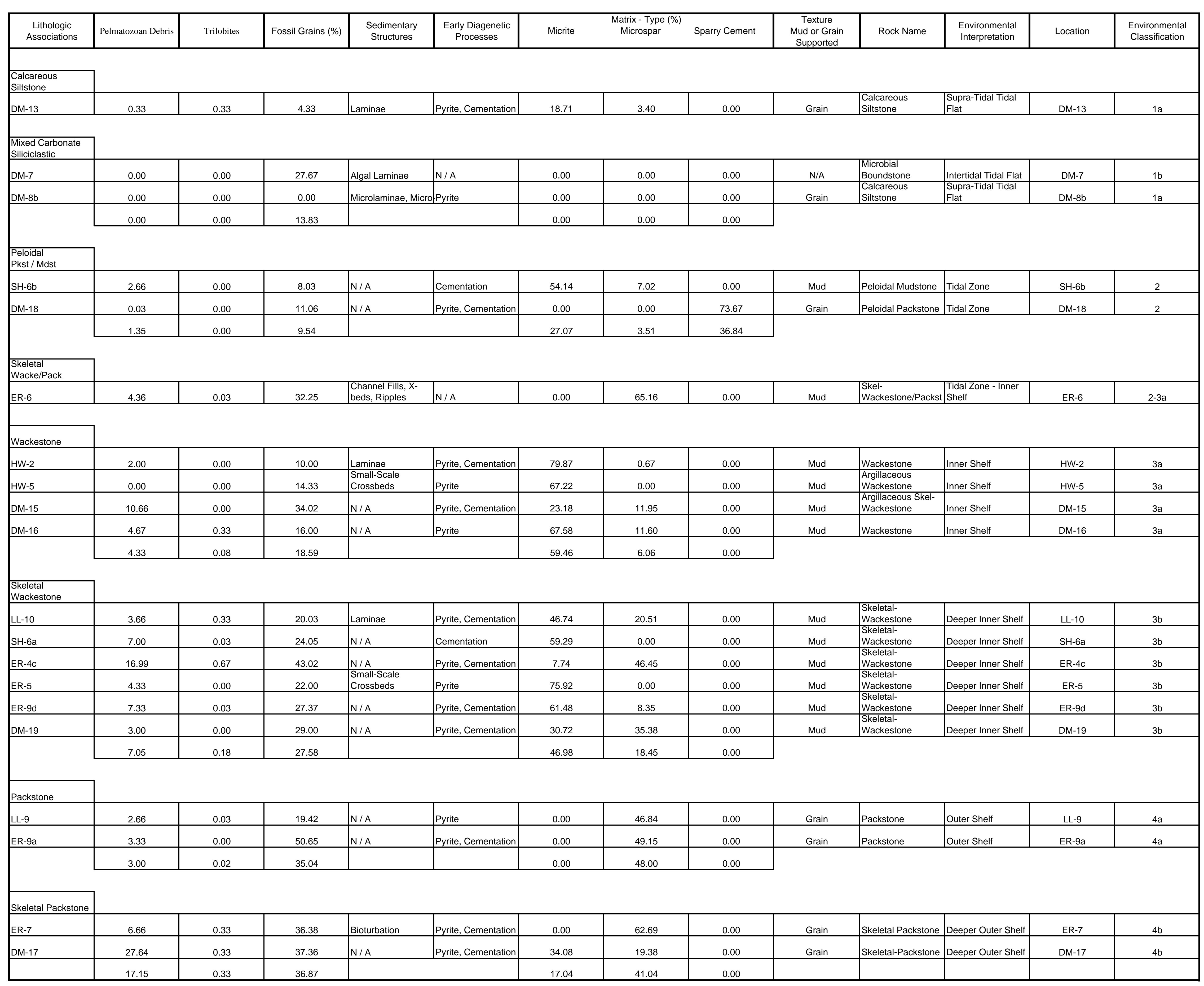


horizontal laminations. There are few body fossils in this unit. The dominant fossil grain types found are bivalves - Wilkingia and brachiopods at 2.00 and 1.67 percent respectively (Table 8 ).

Elevated siliciclastic grain content and the sedimentary structures are important characteristics for interpreting the depositional facies of this association. The key feature linking these strata to tidal flats (7a) is the presence of laminations and the low percent of fossil material (Laporte, 1967; Shinn, 1983). Fossil debris is not uncommon in the supratidal environment but it is generally transported in from adjacent environments during high tide and storm events. Based on this information, this association best fits in the supra-tidal part (A) of the tidal flat model (Fig. 7a).

Peloidal Mudstone / Packstone Association Sh-6b*; DM-18*.

Lithologies within this zone are peloidal mudstones and packstones (Plate 9b). Fossil diversity in this zone is good with; brachiopods - Anthracospirifer, Diaphragmus, Orthotetes, Productus, bivalves - Wilkingia, forams, and pelmatozoans dominating the fossil content.

This association best fits into the tidal flat facies in the depositional model (Fig. 7a). Each of these very different lithologies can occur in a tidal flat realm. Both peloidal mudstones and packstones can be found in restricted or stressed shallow water environments. The primary difference between the two would be the amount of energy present in the environment. The peloidal grainstones would be found in a slightly higher energy environment that would allow more of the finer material to be winnowed away (Halley et al, 1983; Wilson and Jordan, 1983). 


\section{Skeletal Packstone / Grainstone Association}

ER-6*.

This unit is a packstone/grainstone. This unit is highly fossiliferous and dominated by brachiopods: Anthracospirifer, Diaphragmus, Orthotetes, and Productus.

This association represents a transitional environment between the inshore and inner shelf environments. The interbedded nature of this unit varying from channelized limestones to shales suggests regular periods of environmental change. The most probable environment these two would be found in the inshore facies on the depositional model. The varying lithologies found are likely the result from shifting subtidal channels.

\section{Wackestone Association}

HW-2*, 5*; DM-15, 16*.

Lithologies found in this association are wackestones, argillaceous wackestones, and argillaceous skeletal-wackestones. These rocks have a diverse fossil content (Table 8) consisting mainly of brachiopods - Anthracospirifer, Diaphragmus, Orthotetes, bivalves - Wilkingia, forams, and pelmatozoan debris. Siliciclastic influence, quartz and clays, is minimal here. Sedimentary structures include laminae and infrequent small-scale crossbeds.

The wackestones of this association are the dominant lithotypes of the inner shelf (A) facies in this depositional model (Fig. 7b). The inner shelf (A) facies represents normal marine depositional conditions, as indicated by the faunal diversity, in a shallow sub-tidal environment (Kammer and Lake, 2001). The inner shelf (A) is the farthest northward progressing of the subtidal facies. Both the lithotype and the surrounding strata support the inner shelf interpretation (Wilson and Jordan, 1983). The northernmost extent facies marks of this the point of maximum transgression across the study area. 


\section{Skeletal Wackestone Association}

LL-10*; SH-6a*; ER-4c*, 5*, 9d*; DM-19*.

Lithologies within this association are dominated by skeletal-wackestones. These rocks typically contain a very diverse group of taxa: brachiopods - Anthracospirifer, Compositia, Diaphragmus, Orthotetes, Productus; bivalves - Sanguinolites, Wilkingia; gastropods Bellerophon, Straparolus; pelmatozoan and trilobite debris and have very high fossil grain percents in thin section (Table 8). Bioturbation is common with a few small-scale crossbeds found occasionally.

This association is placed in the deeper inner shelf (B) facies on the depositional model (Fig. 7b). The environment here represents deposition during normal marine conditions (Kammer and Lake, 2001) in a deeper water (subtidal) setting. This setting typically has decreased levels of siliciclastics (Table 8) and a relatively high biodiversity (Wilson, 1975, Sellwood, 1978; Wilson and Jordan, 1983).

\section{Packstone Association}

\section{LL-9*; ER-9a*.}

Lithologies within this association are typified by packstones. These rocks have a diverse and prolific faunal assemblage but are dominated by brachiopods (Table 8). Fossils found in these rocks are: brachiopods - Anthracospirifer, Compositia, Diaphragmus; bivalves Wilkingia; gastropods - Straparolus, Bellerphon; pelmatozoan and bryozoan debris. This faunal assemblage is dominated by normal marine taxa indicating deposition under normal marine conditions (Kammer and Lake, 2001). 
This association has been placed in the outer shelf (A) facies on the depositional model (Fig. 7b). The environment here represents deposition during normal marine conditions in a deeper water (subtidal) setting. The lack of sedimentary structures suggests bioturbation by burrowing organisms. The increased faunal diversity and content in these rocks as well as their stratigraphic position in section support their outer shelf (A) interpretation (Sellwood, 1978; Wilson and Jordan, 1983).

Skeletal Packstone Association

ER-7*; DM-17*.

Lithologies in this association are entirely packstones. These rocks have the highest average fossil content of any association (Table 8). The biodiversity of the fauna in this association is among the highest of all the associations. The faunal content suggests deposition under normal marine conditions: brachiopods - Anthracospirifer, Compositia, Diaphragmus, Orthotetes, Productus, bivalves - Wilkingia, pelmatozoan, bryozoan, and trilobite debris (Kammer and Lake, 2001).

This association has been placed in the deeper outer shelf (B) environment on the facies model (Fig. 7b). The very diverse fauna and fossil grain content along with the very low clastic grain influence support the deeper outer shelf (B) interpretation (Wilson, 1975, Sellwood, 1978; Wilson and Jordan, 1983). This association marks the deepest water facies across the study area and is restricted to the southernmost outcrops. 
Mudstone / Shale Association

HW-3; EK-10; ER-4a, b, 6, 8, 9b, 9e; DM-14.

These mudstones and shales are interpreted to represent deposition in a stressed nearshore environment - similar to that of the lower clastic unit. Color variation in these units ranges from red to gray. Interbedding between limestones and shales for these units is common. Fossils found within these units (HW-3; ER-, 8, 9b, 9e; DM-14): brachiopods, bivalves, Ostracodes, which indicate restricted marine or brackish water conditions (McKerrow, 1978; Kammer and Lake, 2001).

Fossiliferous Siliciclastic Association

ER-11, 12.

These shales are interpreted to represent deposition in a stressed nearshore environment where increased siliciclastic influence began to overtake normal marine deposition. These shales range in color from grey to tan and are poorly to mildly fossiliferous. Fossils found in these units are: brachiopods, bivalves, and trilobite hash suggest normal marine deposition with elevated levels of siliciclastics (McKerrow, 1978; Kammer and Lake, 2001).

This association marks the end of the Reynolds transgressional event and the initial stages of Mauch Chunk deposition. 
Non-fossiliferous Siliciclastic Association

HW-4, 6; LL-upper covered interval; CH-4, 6; SH-7, 8; EK-12, 13; ER-10, 13; DM- upper covered interval.

Lithologies found in the non-fossiliferous siliciclastic lithofacies association are the sandstones and shales above the Reynolds Limestone at the top of each section. Shales found in these rocks are typically red and devoid of fossil material. Sandstones, all of which lack fossil material, display crossbedding with small-scale scoured bases.

This lithologic association displays characteristics typical of a fluvial environment (Cant, 1982). This association is included in the fluvial facies of the depositional model (Fig. 7a) and marks the beginning of the Mauch Chunk Clastic Wedge and a major regressional phase in the basins history.

\section{DEPOSITIONAL MODEL FOR ENVIRONMENTAL INTERPRETATIONS}

This model blends characteristics of both the carbonate ramp and shelf models (Ahr, 1973; Wilson, 1975). Variables used in the designation of the units into various environmental categories include: relative hydrodynamic energy levels, relative water depth, circulation, terrigenous influx of sediments, the abundance, type, and size of biota found, and the stratigraphic relationship between the various units being studied (Table 8).

The suggested paleoenvironmental model for the Late Mississippian Reynolds depositional system is shown in Figures 7a, b. Six facies associations have been identified that characterize environmental conditions on a broad, shallow, low-angle interior carbonate shelf system (Fig. 7a, b). The marginal marine facies spans the boundaries of several other associated 
subenvironments. This was done intentionally in order to characterize a significant amount of strata below the study interval, but still allow environmental interpretations to be made. The marginal marine facies is a gradational interfingering zone of other facies. Fluvial facies are the most landward facies in this model. The tidal flat facies represents the most restrictive depositional conditions of any carbonate depositional environment in this study. The tidal zone is a transitional environment that is regularly influenced by tidal currents. The inner and outer shelf facies are named based exclusively on relative water depth for this model. Both are considered shallow - with the outer shelf the deeper of the two sub-tidal facies.

The Reynolds Limestone model adapted here consists of quiet water shallow, nearshore, marine conditions. The paleo-slope of the sea floor was very low, similar to that of an epicontinental sea. Low to moderate tidal currents were the dominant hydrodynamic influence on the environmental distribution in the model. Transition between facies across the study area resulted from the loss of energy as tides and currents crossed the broad, low-angle shelf. Boundaries between facies are gradational and therefore can be difficult to place precisely within the model, or on the outcrop.

\section{SEA LEVEL FLUCTUATIONS AND FACIES MAPS}

Sea level fluctuations and facies migrations were mapped based on cross-sections and correlations (Plate 11) made across the study area. Maps were created to show changes in environmental conditions through time beginning at the base of section. Figure 8 illustrates all the paleoenvironmental factors (paleoshoreline, source area, subsidence rates, and direction of sea level rise) considered in making the time-facies maps. A better illustration of the influence of subsidence in the study area is Figure 9. This map illustrates the thickness variation from the 


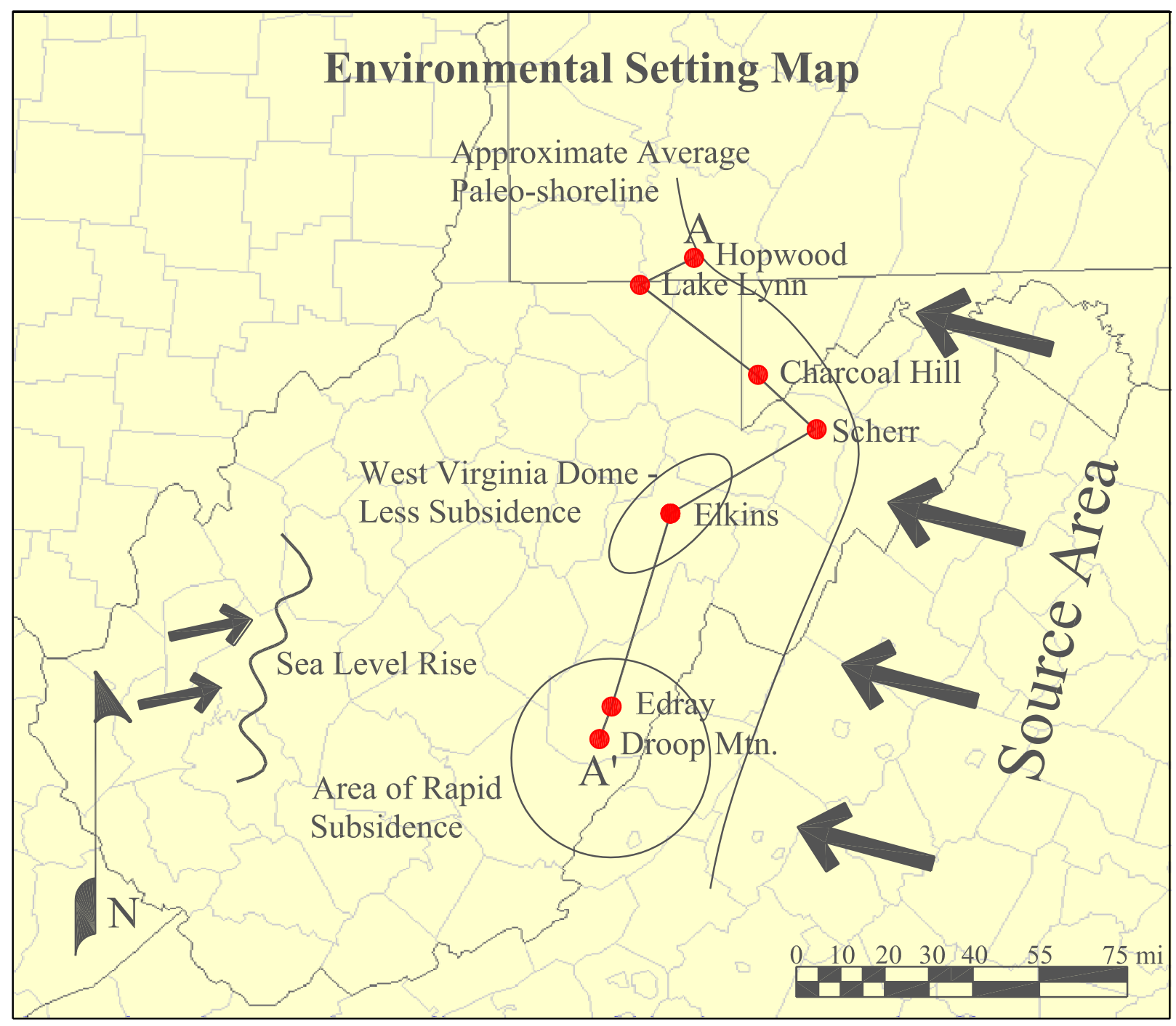

Figure 8. This map illustrates the major environmental conditions influencing depositional settings during this time. 


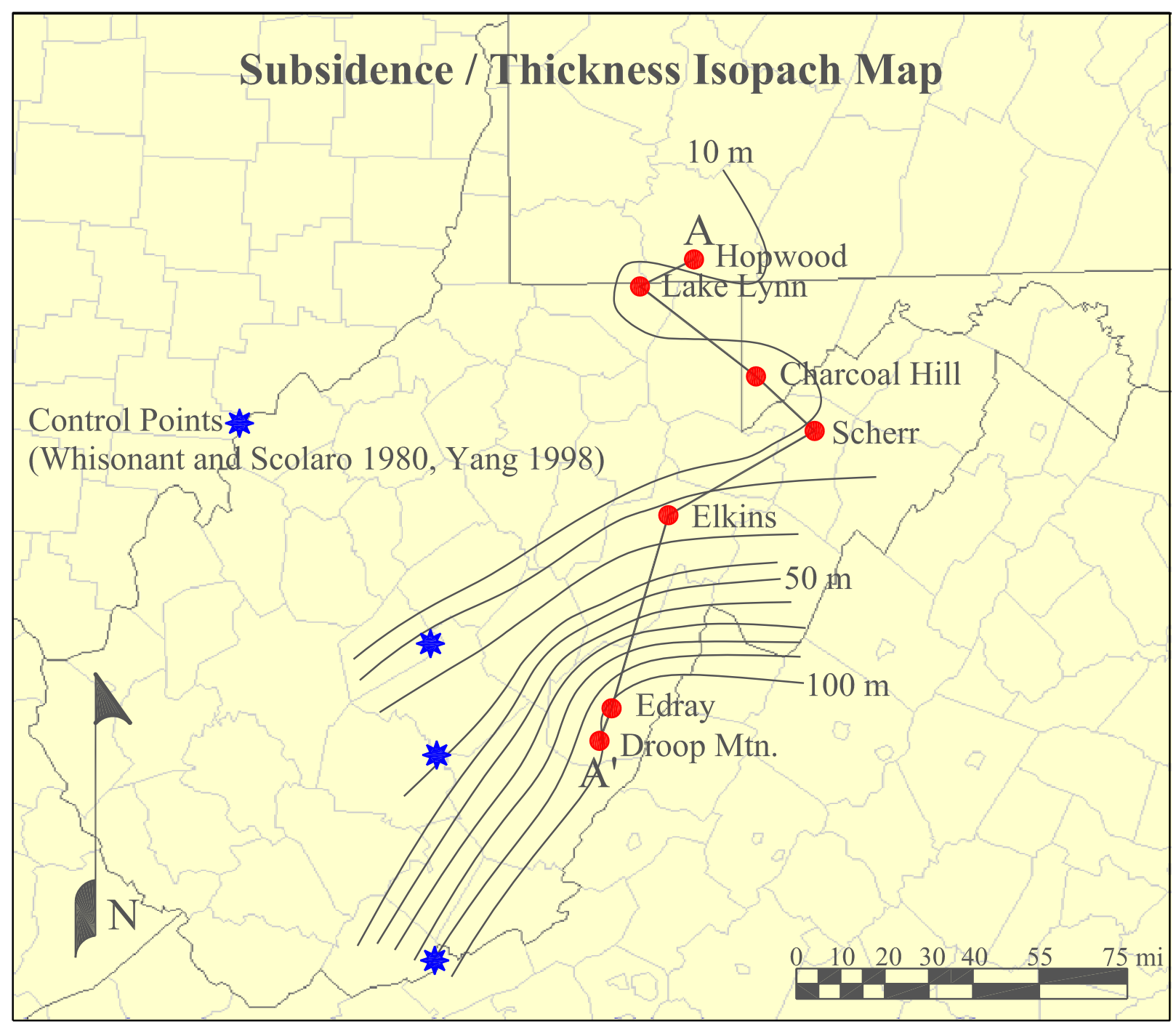

Figure 9. This figure shows the thickness variation across the study area between the top of the Greenbrier and the top of the Reynolds (North - 5.25 m. South - $117.22 \mathrm{~m}$ ). Assuming constant sedimentation rates through time a much higher subsidence rate must have occurred in the south (22.33 x faster). The source area is to the east of the study area. The fact that the southern outcrops maintain normal marine conditions for longer periods is further evidence supporting higher subsidence rates in the south. 
top of the Greenbrier Limestone to the top of the Reynolds Limestone across the study area. Some control points have been included to provide more accurate isopach lines. Assuming a constant sedimentation rate, the southern study area must have been subsiding much more rapidly than the northern section (22.33 times faster). Four separate "snapshots" in time (T0-T3) are illustrated.

Map of time T0 (Figure 10) reflects paleoenvironmental conditions before the first major transgression. The top of the Lillydale shale was used as the timeline. All localities at this time were in a marginal marine setting undergoing a period of transition between carbonate and clastic environments.

The second map at time T1 (Figure 11) represents the first major marine transgression. The base of the Reynolds Limestone was used to illustrate paleoenvironmental conditions. A major change in facies resulting from a rise in sea level is apparent. Paleoshoreline at this time shows an embayment in the middle part of the outcrop belt. When compared to Figure 2 from McKinney and Gault (1989), the paleoshoreline illustrated here shows a very similar trend.

The map of time T2 (Figure 12) shows maximum transgression. It can be seen from this map that the inner shelf facies is the deepest of the normal marine facies to prograde into the northern sections. Paleoshoreline again shows an embayment in the middle to upper part of the outcrop belt. The notable difference between times T1 and T2 is that the paleoshoreline of T2 is not as apparent as that of T1.

The beginning of the regression is at time T3, which marks the initiation of deposition of the Mauch Chunk clastic wedge (Figure 13). This marks a time when marginal marine and fluvial conditions once again dominated the study area. 


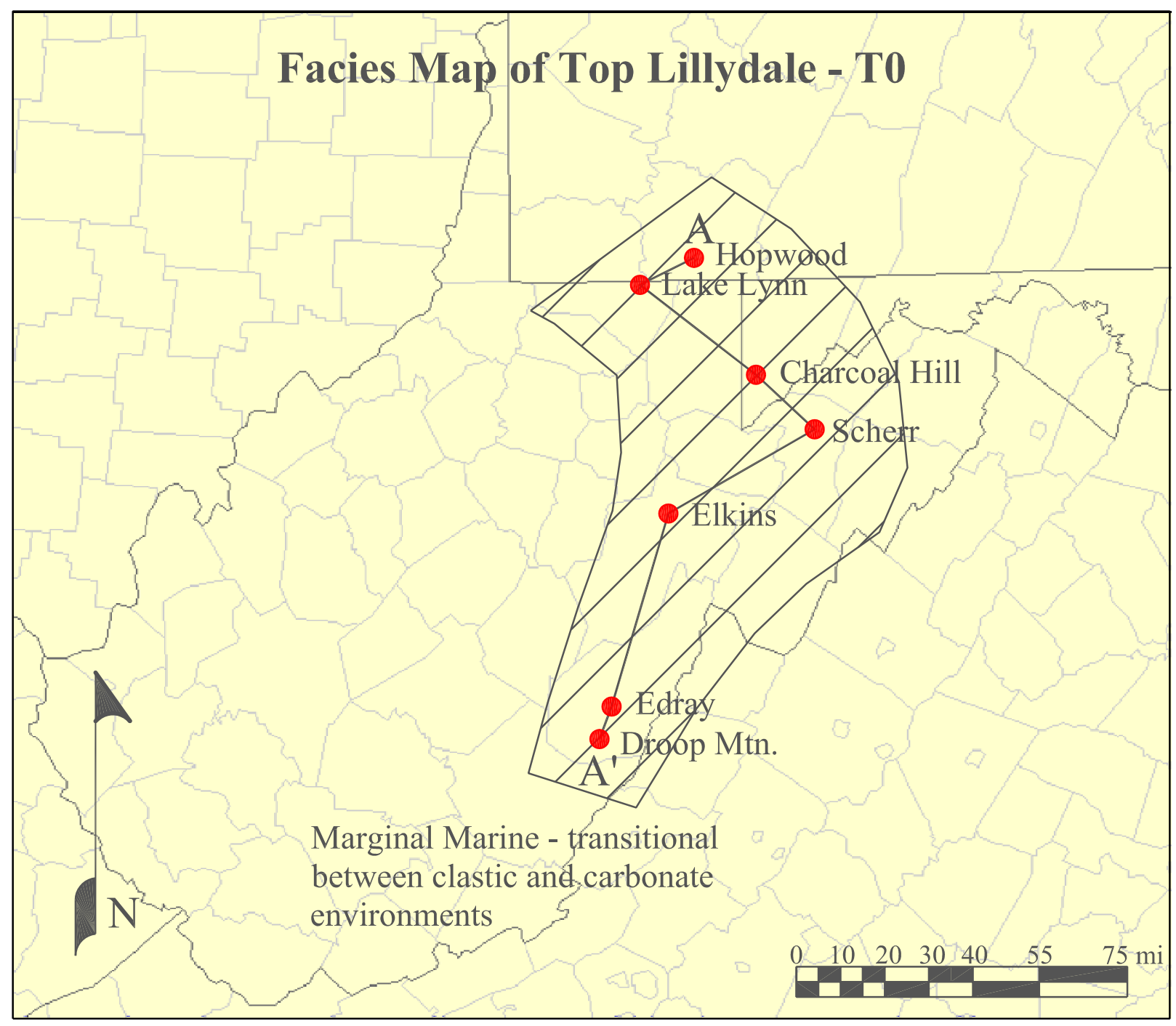

Figure 10. This figure shows the paleoenvironmental conditions across the study area at the top of the Lillydale shale or at time T0. 


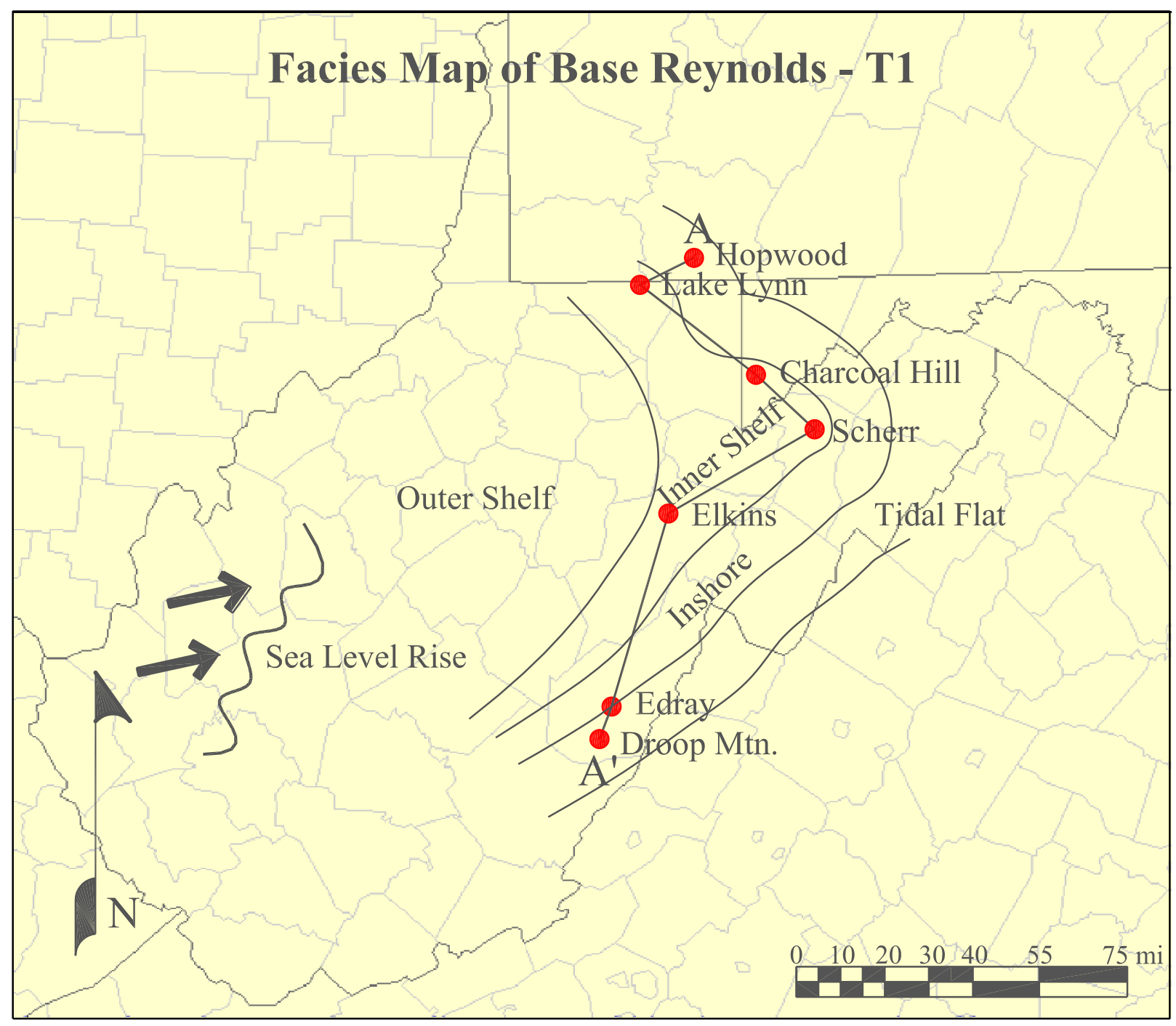

Figure 11. This figure shows facies migration at the beginning of the base Reynolds transgression time $\mathrm{T} 1$. 


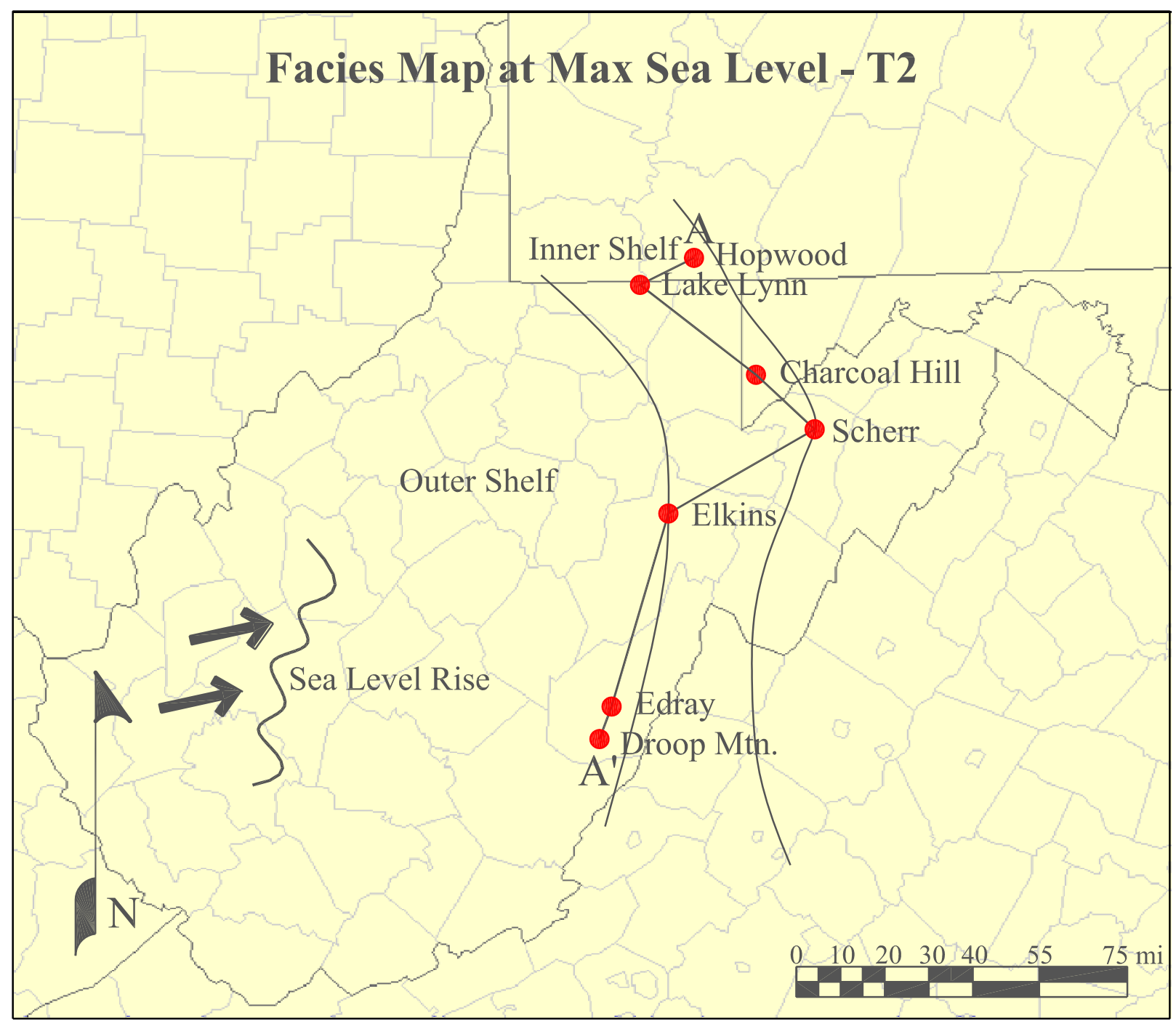

Figure 12. This figure illustrates the facies during the maximum point of transgression at time $\mathrm{T} 2$. 


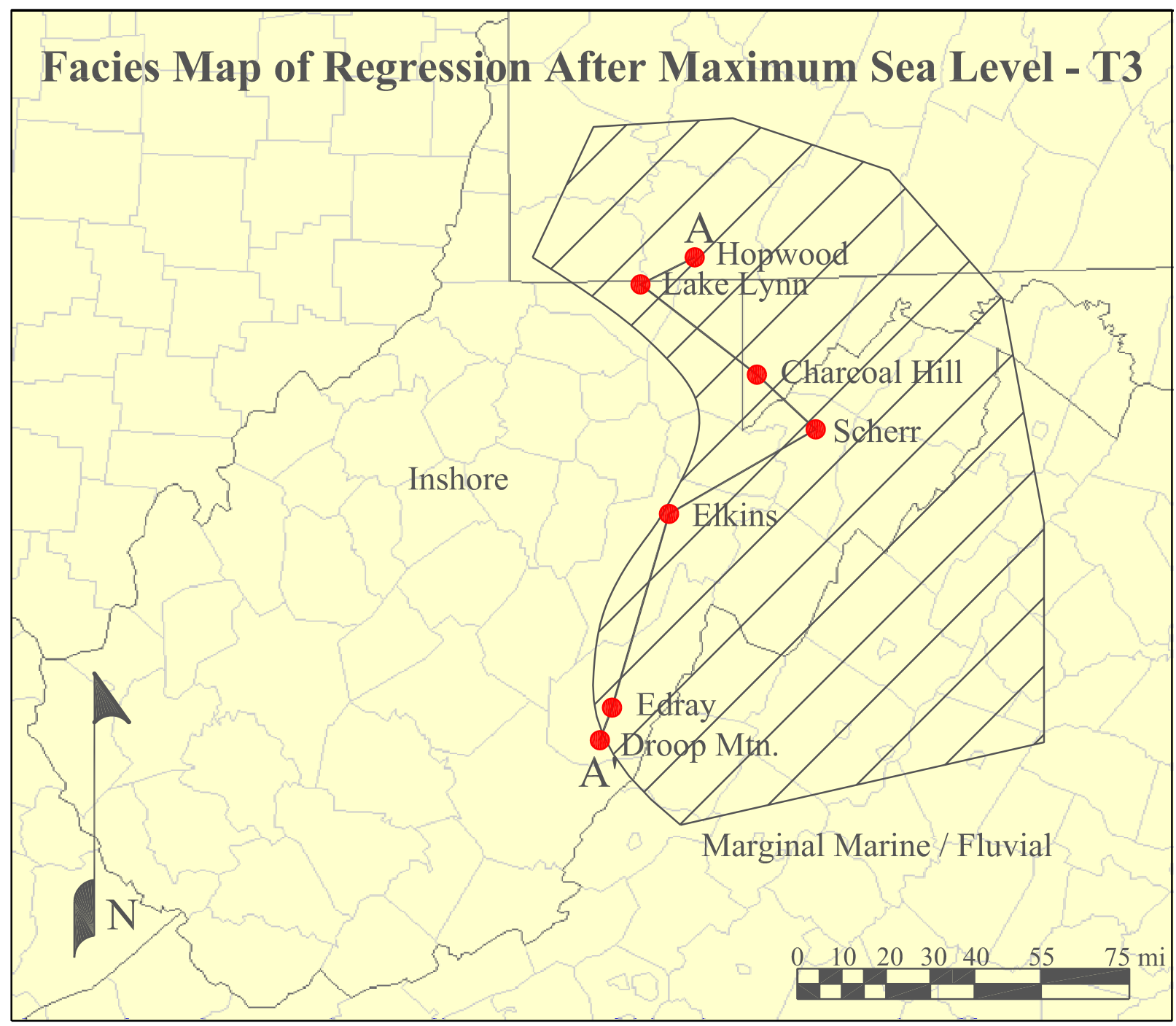

Figure 13. This figure shows the change in facies after the maximum transgression at the beginning of the regression into the Mauch Chunk clastics at time T3. 
The paleoshoreline orientation throughout Reynolds deposition suggests this unit could be used as a timeline to aid in reconstructing the history of the Appalachian Basin during Late Mississippian times. Transgressions and regressions during this time were basically perpendicular (east to west) to the overall paleoshoreline trend (north to south). This paleoshoreline trend would cause each fluctuation in sea level across the study area to occur at approximately the same rate and time. The result is a time synchronous datum that corresponds to the Reynolds Limestone along outcrop strike.

\section{CROSS-SECTIONS AND CORRELATIONS}

Detailed cross-sections were created in AutoCAD and used for outcrop descriptions (Appendix 1). These cross-sections were then incorporated into one drawing, stacked in stratigraphic order, and arranged from North to South across the study area (Plate 11). The correlations for the outcrops were arranged with the base of the Reynolds as a horizontal datum. In all but the Droop Mountain sections, the Reynolds is considered the first normal marine limestone above the top of the Greenbrier.

Environmental interpretations were made based on relative hydrodynamic energy levels, relative water depth, circulation, terrigenous influx of sediments, the abundance, type, and size of biota found, and the stratigraphic relationship between the various units being studied. Sea level curves were applied to each outcrop based on these environmental interpretations. Correlations were then made based on facies relationships and sea level fluctuations. Large-scale events (sea

level fluctuations) were chosen for correlation instead of litho- or biofacies because carbonate systems are very complex and would be much more difficult to correlate over great distances (Wilson, 1975; Al-Tawil et al, 2003). 


\section{DISCUSSION}

The outcrop search for this project began by reviewing previous research by former masters and doctoral students. Particularly helpful in locating outcrops were Tissue (1986); Carney (1987); Christopher (1992); and Lake (1998). These studies were used for reference and as a springboard for the current research. As field data were collected, it became apparent some of the previous investigations were seriously flawed. Two major problems arose when crossreferencing data collected for this study with that of Carney's (1987) and Christopher's (1992) dissertations.

The first problem was identified in a preliminary examination of the Edray outcrop conducted on a field trip for Advanced Paleontology (04/17/04). Christopher's dissertation was used for reference as we walked through his measured section. Investigation of his measured section Unit 11, his Webster Springs Sandstone (unit 2d, Plate 11), yielded numerous marine fossils, particularly brachiopods (Plate 3c). As described in the Webster Springs Sandstone stratigraphic overview of his dissertation (Christopher, 1992), the true Webster Springs Sandstone is a terrestrial (fluvial) sandstone. The sandstone exposed at Edray is therefore not equal to the Webster Springs. The resultant effect on the remainder of his measured section is significant. The miscorrelation of the Webster Springs Sandstone caused all other lithologic units to be downshifted in stratigraphic position. This would explain the over-thickened Bickett Shale and Reynolds limestone sections as well as the absence of the Glenray Limestone at this outcrop in his dissertation. The most likely explanation for this is the lateral discontinuity of the Bickett Shale and Webster Springs Sandstone, possibly caused by a localized forced regression, between the Droop Mountain and Edray sections. Bearing this in mind, it is entirely possible the Glenray and Reynolds Limestones are lying in direct contact with one another at this outcrop. 
Without the separation of the two units by a clastic interval, differentiation of these units becomes very difficult if not impossible.

A second problem was identified during the field investigation of the Scherr outcrop (06/08/04). The measurement of this outcrop began based on the stratigraphic position of the Reynolds Limestone as identified by Carney’s (1987) dissertation. Upon returning to the laboratory to review the data collected, it quickly became apparent that this section did not match-up lithologically or stratigraphically with any other sections measured for this study. After reviewing the literature, it was found that Carney had miscorrelated the uppermost Greenbrier Limestone (Wymps Gap Member) to the Reynolds Limestone at the Scherr locality. The overall effect of the miscorrelation at this outcrop was significant. Shifting the position of the Reynolds Limestone by Carney to a lower stratigraphic position caused a significant over thickening of the Reynolds here. A second trip was made to re-measure the section with the corrections made for the miscorrelation of the Reynolds with the Wymps Gap Limestone. Revised measurements and descriptions were used for Appendix 1 and Plate 11.

Multivariate quantitative methods were attempted with the data from this research. A discussion of the methodology and results are available in Appendix 3.

Large-scale applications of this study relate the sea level fluctuation patterns related here to extra-basinal regional settings. The Reynolds Limestone is regionally correlative to the Glen Dean Limestone of the Illinois Basin (Patchen et al., 1985a, b). Smith and Read (1999) found the base of the Glen Dean Limestone to be time correlative with the Early Namurian (middle 
Chesterian). Based on the correlation of Patchen et al. (1985a, b), it can be inferred that the base of the Reynolds Limestone is also time correlative with the base of the Namurian.

Numerous studies have been conducted in the Illinois Basin on the Upper Mississippian strata found there. Similar stacking patterns from this and many other Upper Mississippian basins suggest global-scale mechanisms were influencing deposition during this time (Smith and Read, 2001). Numerous hypotheses have been offered to explain the Upper Mississippian sea level fluctuations. Based on the over thickened southern sections (Plate 11) it is apparent there were some tectonically related influences on deposition at this time (Al-Tawil et al., 2003). The isopach map of strata in the Reynolds created for this study (Fig. 9) matches closely with the isopach map of Al-Tawil et al. (2003) created for the Upper Mississippian Greenbrier Limestone in southern West Virginia (Fig. 14). Reactivated tectonism, flexural responses to tectonic loading, and forebulge migration are all relevant tectonically related factors explaining these sea level fluctuations. However, it has been documented that the onset of glacial activity during the Upper Mississippian is responsible for these sea level fluctuations (Smith and Read, 1999, 2000, 2001; Al-Tawil, et al., 2003). The regularity of the depositional facies stacking patterns further argues against tectonic controls and support glacial influences (Smith and Read, 2001). Major continental ice sheets began to form on Gondwana during late Visean / early Namurian times (Smith and Read, 1999; Al-Tawil et al., 2003). This resulted in a series of small-scale transgressions (glacial meltback) and regressions (increased periods of glaciation) during the Glen Dean / Reynolds depositional period caused by the onset of glaciation in the early Namurian (Smith and Read, 2000) (Fig. 15). Facies distribution across the study area and their vertical stacking patterns in outcrop reflect similar sea level fluctuations (Plate 11) to those of Figure 15. Of important note on Figure 15, is the major change in the magnitude of sea level 


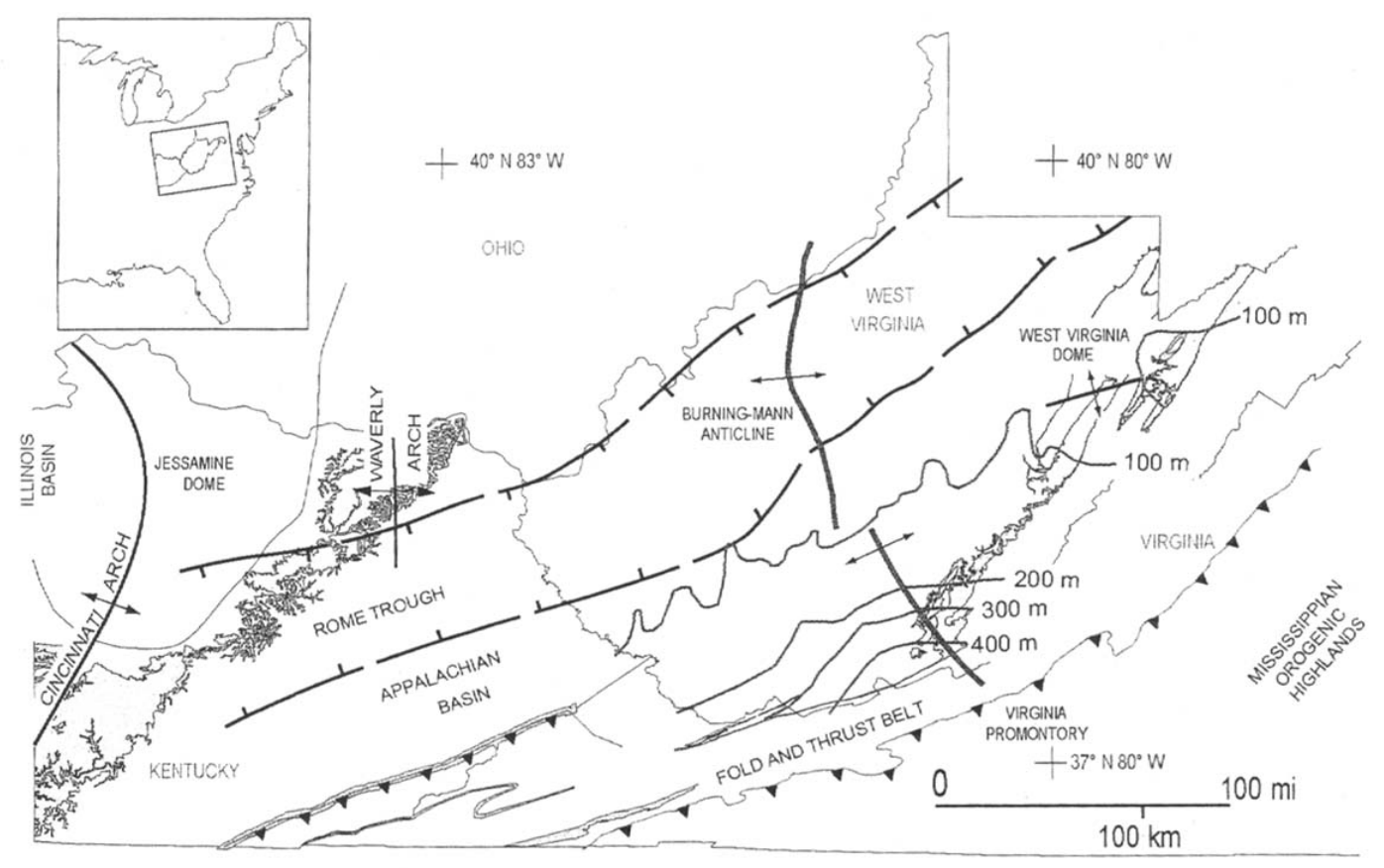

Figure 14. Isopach thickness map of the Upper Mississippian Greenbrier Limestone from southern West Virginia. Notice the increasing thickness toward the southeast and trend of the isopach lines (Al-Tawil et al., 2003). 


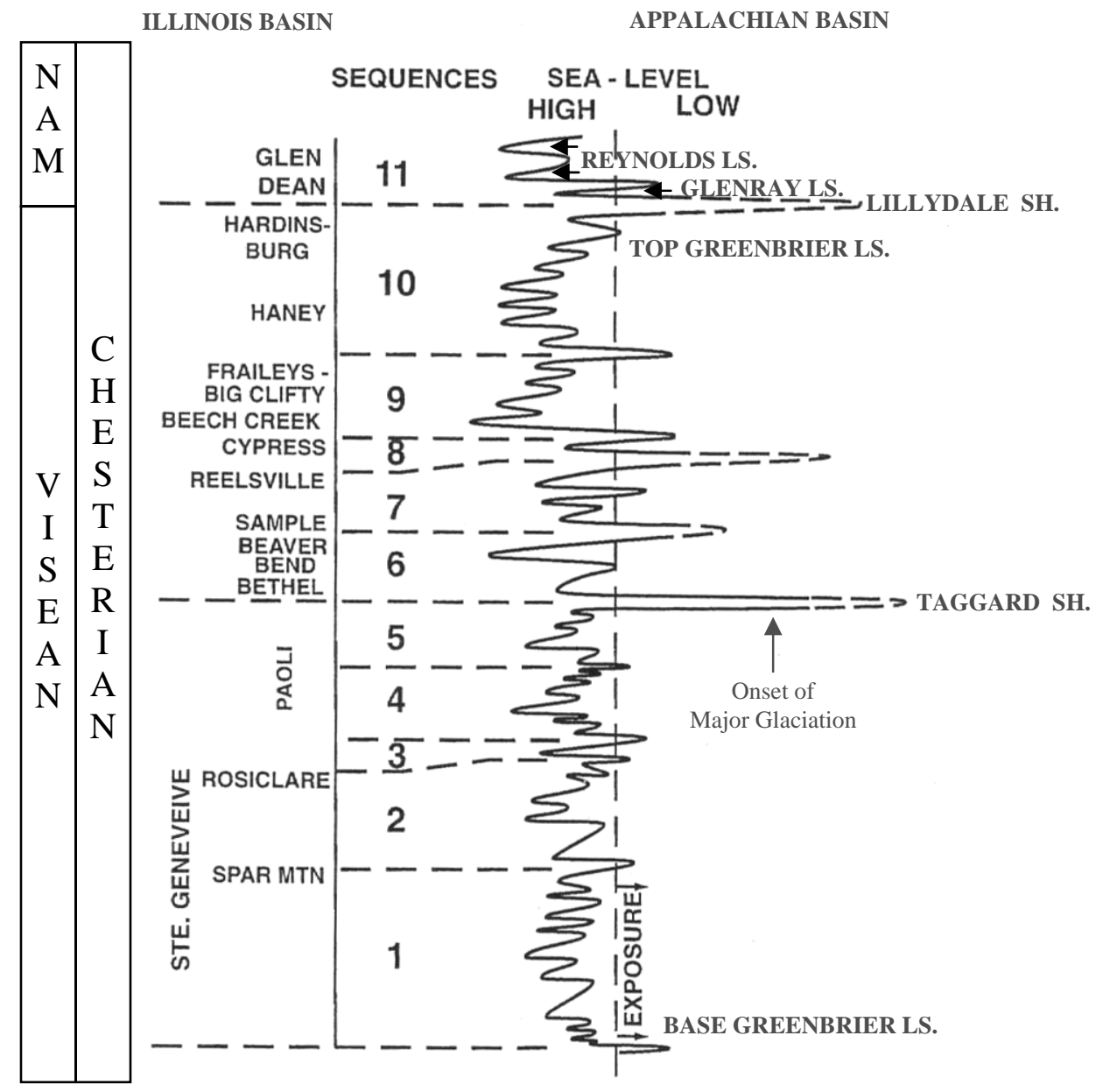

Figure 15. Composite sea level curve for early and middle Chesterian (late Visean to early Namurian) in the Illinois Basin. Notice the increase in the amplitude of sea level fluctuation after the onset of major glaciation (Smith and Read, 1999; Al-Tawil et al., 2003). 
fluctuations after the onset of major glaciation. This is further evidence supporting Late Paleozoic glaciation (Smith and Read, 2000).

Sequence stratigraphic principles were employed to define the maximum sea level rise identified in Plate 11. Al-Tawil et al. (2003) defined the strata in this study interval as the Highstand Systems Tract (HST). The transgressive / regressive changes in sea level are likely the result of periods of glacial advance and retreat during the highstand. These small-scale transgressive and regressive episodes equate to small, meter-scale parasequences, which Smith and Read (1999) use as timelines for intrabasinal correlation and to aid in identifying larger-scale sequences. Overall, the Reynolds depositional interval (top Greenbrier to base Mauch Chunk) represents only a portion (HST) of a large-scale sequence.

The stratigraphic / facies relationships between the Reynolds and Glenray limestones are outside the scope of this study and are mentioned here only as a suggestion for further research. Figure 16 illustrates the regional relationship between the Reynolds and Glenray limestones of West Virginia and the Cove Creek Limestone of Virginia. As shown, a clastic tongue separates the Reynolds and Glenray limestones in West Virginia but is absent in Virginia. A controversy with the terminology and correlation between these units in West Virginia arises where a distinct clastic unit does not separate the two. Currently the Reynolds (a fossiliferous wackestone) and the Glenray (an argillaceous laminated mudstone) limestones are identified based on type sections and their lithologic characters at these type sections. Carbonate environments are very complex, biologically controlled systems (Wilson, 1975). These environments change quickly causing rapid facies changes in short distances. This can result in drastically different lithologies from the same time period and depositional system being placed in close proximity to one another such as the Reynolds and Glenray. Therefore, the separation of closely related 


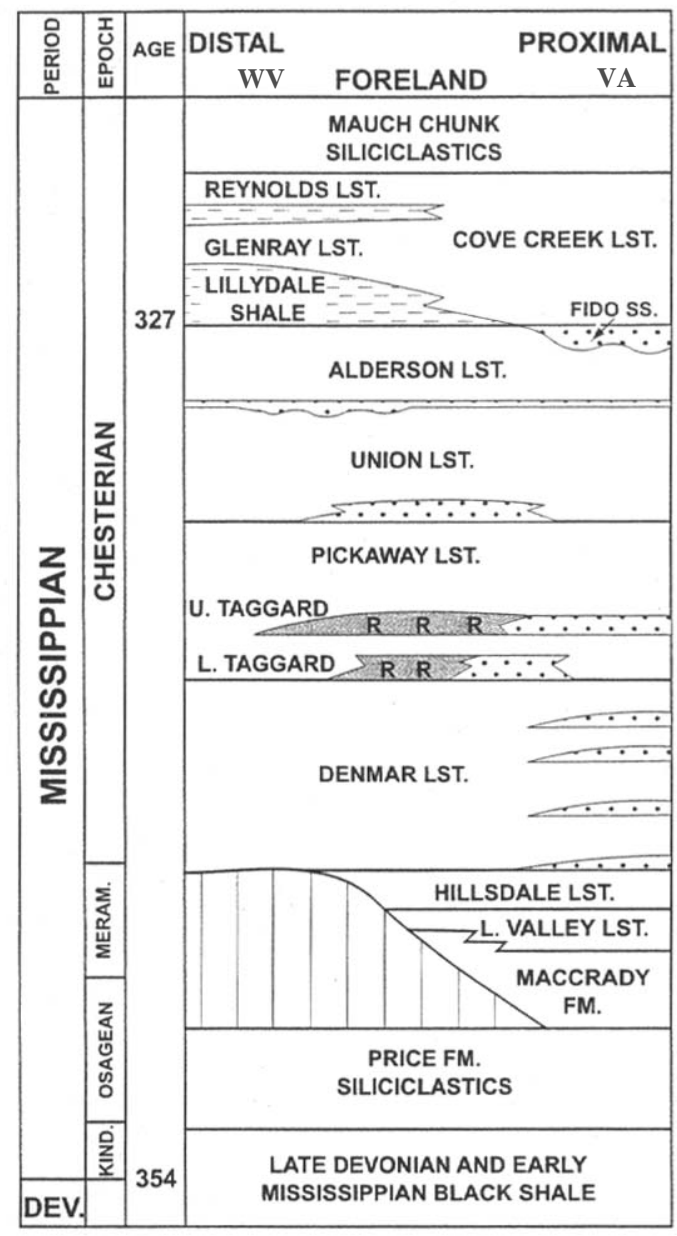

Figure 16. Separation of the Reynolds and Glen Ray Limestones in West Virginia by a clastic tongue makes division of the two possible. In Virginia, where there is no such clastic division the two are grouped into the Cove Creek Limestone (Al-Tawil et al., 2003). 
limestones with different histories based on one depositional facies or type section is not appropriate. If it is determined the two cannot be differentiated, the current accepted stratigraphic nomenclature should be applied and the unit called the Reynolds Limestone. Further study should be conducted to address this matter.

\section{CONCLUSIONS}

The Reynolds Limestone is regionally correlative to the Glen Dean Limestone of the Illinois Basin. The correlation between the Glen Dean and Reynolds Limestone intervals demonstrates that large-scale glacial effects were controlling eustatic sea level fluctuations in Upper Mississippian times. Specifically, an early onset glacial event is recorded by the Lillydale Shale at late Visean (middle Chesterian) times and interglacial periods by the Reynolds and Glenray limestones in the early Namurian (middle Chesterian).

Facies analysis of the Reynolds Limestone suggests it was formed during several smallscale transgressive and regressive episodes but overall represents normal marine depositional conditions in a Highstand Systems Tract. These small-scale sea level fluctuations record the beginning of an Upper Mississippian ice age. Regressions correspond to glacial periods and transgressions to interglacial meltback events.

The Reynolds Limestone is over thickened in the southern section. This results largely from apparent structural controls on the overall basin geometry causing rapid subsidence in southern West Virginia. Structural controls, however, did not cause eustatic sea level fluctuations. This suggests that the relative water depth for the southern outcrops was greater, thus allowing normal marine deposition to occur without interruption for longer periods of time. 
The stratigraphic and geographic position of these units in the cross-section (Plate 11) is also of note. All of the outer shelf normal marine units are found in the lower half of the upper limestone interval and only in the southern sections (Plate 11).

The Reynolds Limestone can be considered a time correlative unit. This is based on the paleoshoreline orientation ( north-south) of the Reynolds and its relationship to deeper basinal settings and the direction of sea level rise and fall ( east-west). The result of such an orientation would be sea level fluctuations occurring nearly contemporaneously across the entire depositional system.

The Webster Springs Sandstone of the Droop Mountain section that separates the Glenray from the Reynolds Limestones appears to represent a forced regressional event that is laterally discontinuous or gradational into another facies.

Where a distinct clastic unit does not separate the Reynolds and Glenray limestones, it is difficult if not impossible to differentiate the two. In order to conclusively resolve the correlation issues between the Reynolds and Glenray Limestones, further investigation needs to be conducted. Correlating well cores and outcrops would provide much greater control on the varying thicknesses of both units and better track the stratigraphic relationship and possible continuity between the two.

In the future, the use of binary (presence / absence) data will require more thorough sampling of units (bulk sampling with specimen counts) within the Reynolds Limestone. In order for this analysis technique to be employed with better results, the sampling methods of the study need to be designed appropriately. The primary source of data used in this study came from thin sections. Visual inspection was used when selecting which units to have thin sections 
made of. Future work should sample both fossiliferous and non-fossiliferous units at more detailed bed-by-bed resolution to avoid sample bias. 


\section{References}

Ahr, W., M. 1973. The Carbonate Ramp: An Alternative to the Shelf Model: Gulf Coast Geological Society, 23:221-225.

Al-Tawil, A, Wynn, T. C., and Read, J. F., 2003. Sequence Response of a Distal-to-Proximal Foreland Ramp to Glacio-Eustasy and Tectonics: Mississippian, Appalachian Basin, West Virginia-Virginia, U.S.A.: SEPM Publication no. 78, p. 11-34.

Arkle, T., Beissel, D. R., Larese, R. E., Nuhfer, E. B., Patchen, D. G., Smosna, R. A., Gillespie, W. H., Lund, R., Norton, C. W., and Pfefferkorn, H. W., 1979. The Mississippian and Pennsylvanian (Carboniferous) Systems in the United States - West Virginia and Maryland: Geological Survey Professional Paper 1110 - D.

Boardman, M. R., Carney, C., and Bergstrand, P. M., 1993. A Quaternary Analog for Interpretation of Mississippian Oolites: AAPG Studies in Geology 35:161-173.

Boggs, S., 1987. Principles of Sedimentology and Stratigraphy: Marginal-Marine Environments: 12:395-451.

Brezenski. D. K., 1984. Dynamic Lithostratigraphy and Paleoecology of Upper Mississippian (Chesterian) Strata of the Northcentral Appalachian Basin: University of Pittsburgh, PhD Dissertation, 132 p.

Brezinski, D. K., 1989 a. Late Missippian depositional patterns in the north-central Appalachian Basin, and their implications to Chesterian heirarchail stratigbraphy: Southestern Geology, v. 30, p. 1-23.

Brezinski, D. K., 1989 b. The Mississippian system in Maryland: Maryland Geological Survey, Report of Investigations no. 52.

Busans, J. W., 1974. Paleontology and Paleoecology of the Mauch Chunk Group in Northwestern West Virginia: Bowling Green State University, Masters Thesis, 388 p.

Busanus, J. W., and Hoare, R. D., 1991. Bivalves (Mollusca) from the Mauch Chunk Group (Mississippian, Chesterian) of Northern West Virginia and Southwestern Pennsylvania: 65:3:465-480. 
Cant, D. J., 1982. Fluvial Facies Models and Their Application: AAPG Memoir 31:115-137.

Carney, C., 1987, Petrology and diagenesis of the Upper Mississippian Greenbrier Limestone in the Central Appalachian basin and of the Lower Carboniferous Great Limestone in Northern England: West Virginia University, Dissertation, 400 p.

Carney, C., and Smosna R., 1989, Carbonate deposition in a shallow marine fulf, the Mississippian Greenbrier Limestione of the central Appalachian basin: Southeastern Geology, v. 30, p. 25-48.

Christopher, C. C., 1992, Marine paleocommunities and paleoenvironments of the Upper Mississippian Bluefield Formation in southern West Virginia: West Virginia University, Dissertation, 447 p.

Corbitt, L. B., 1986. The petrology and stratigraphy of the Reynolds Limestone member of the Bluefield Formation (Mississippian) in southeastern West Virginia, East Carolina University, Masters Thesis, $106 \mathrm{p}$.

Donaldson, A. C., and Shumaker, R. C., 1981. Late Paleozoic molasse of central Appalachians: Geological Addociation of Canada Special Paper no. 23. p. 99-124.

Dunham, R. J., 1962. Classification of Carbonate Rocks According to Depositional Texture: AAPG Memoir 1: 108-121.

Feldmann, R. M. ed.,1996. Fossils of Ohio: Ohio Department of Natural Resources Division of Geological Survey. Bulletin 70. 577p.

Halley, R. B., Harris, P. M., and Hine, A. C., 1983. Bank Margin: AAPG Memoir 33:463-506.

Harwood, G., 1988. Microscopical techniques: II. Principles of sedimentary petrography: Techniques in Sedimentology, ch. 5, p. 108-173.

Henry, T. W., and Gordon, M. 1992. Middle and Upper Chesterian Brachiopod Biostratigraphy, Eastern Appalachians, Virginia and West Virginia: Oklahoma Survey Circular 94:1-21.

Hickok, W. O., and Moyer, F. T., 1971. Geology and mineral resources of Fayette County, Pennsylvania: Pennsylvania Topographic and Geologic Survey. County Report C26. 530 p. 
Humphreville, R. G., 1981. Stratigraphy and Paleoecology of the Upper Mississippian Bluefield Formation: West Virginia University, Masters Thesis, 230 p.

Inden, R. F. and Moore C. H., 1983. Beach Environment: AAPG Memoir 33:215-265.

Kammer, T. K, and Ausich, W. I., 1987. Aerosol Suspension Feeding and Current Velocities: Distributional Controls for Late Osagean Crinoids. Paleobiology, 13:379-395.

Kammer, T. K., and Lake, A. M., 2001. Salinity Ranges of Late Missippian Invertebrates of the Central Appalachian Basin: Southeastern Geology, v. 40, no. 2, p. 99-116.

Lake, A. M., 1998, Marine paleoecology of Upper Mississippian strata in northern West Virginia and southwestern Pennsylvania: West Virginia University, Masters Thesis, 95 p.

Laporte, L. F., 1967. Carbonate Deposition Near Mean Sea-Level and Resultant Facies Mosaic: Manilus Formation (Lower Devonian) of New York State: AAPG Memoir 51:73-101.

Manspeizer, W., 1958. The Bluefield Group - Mauch Chunk Series in Southeastern West Virginia: West Virginia University, Masters Thesis, 96 p.

McKerrow, W. S. ed., 1978. The Ecology of Fossils: Carboniferous p. 146.

McKinney, F. K., and Gault, H. W., 1989. Paleoenvironment of Late Mississippian fenestrate bryozoans, eastern United States: Lethaia, v. 13, p. 127-146

Moore, R. C. ed., 1959. Treatise on Invertebrate Paleontology: Part O. Arthropoda 1. p. O1O560. Geological Society of America and University of Kansas Press.

Moore, R. C. ed., 1965a. Treatise on Invertebrate Paleontology: Part H. Brachiopoda. Vol 1. p. H1-H522. Geological Society of America and University of Kansas Press.

Moore, R. C. ed., 1965b. Treatise on Invertebrate Paleontology: Part H. Brachiopoda. Vol 2. p. H523-H927. Geological Society of America and University of Kansas Press.

Moore, R. C. ed., 1969a. Treatise on Invertebrate Paleontology: Part N. Mollusca 6 Bivalvia. Vol. 1. p. N1-N489. Geological Society of America and University of Kansas Press.

Moore, R. C. ed., 1969b. Treatise on Invertebrate Paleontology: Part N. Mollusca 6 Bivalvia. Vol. 2. p. N491-N952. Geological Society of America and University of Kansas Press. 
Moore, R. C., Lalicker, C. G., and Fischer, A. G., 1952. Invertebrate Fossils. 776 p.

Patchen, D.G., Avary, K.L., and Erwin, R.B. Regional Coordinators. 1985a. Southern Appalachian Region: Correlation of Stratigraphic Units of North America (COSUNA) Project. American Association of Petroleum Geologists. Tulsa, Oklahoma.

Patchen, D.G., Avary, K.L., and Erwin, R.B. Regional Coordinators. 1985b. Northern Appalachian Region: Correlation of Stratigraphic Units of North America (COSUNA) Project. American Association of Petroleum Geologists. Tulsa, Oklahoma.

Presley, M. W., 1977. A Depositional Systems Analysis of the Upper Mauch Chunk and Pottsville Groups in Northern West Virginia: West Virginia University, PhD Dissertation, 157 p.

Price, P. H., 1929. Pocahontas County Report: West Virginia Geological and Economic Survey. County Report. 866 p.

Reger, D. B., et al, 1918. County reports of Barbour and Upshur counties and the eastern portion of Randolph county. West Virginia Geological and Economic Survey. County Report. 867 p.

Reger, D. B., et al, 1920. County reports of Webster county and a portion of Mingo district, Randolph County, and south of the Valley Fork of Elk River. West Virginia Geological and Economic Survey. County Report. 682 p.

Reger, D. B., et al, 1924. County reports of Mineral and Grant Counties: West Virginia Geological and Economic Survey. County Report. 866 p.

Rholf, F. J., 1998. Numerical taxonomy system of multivariate statistical programs: manual. State University of New York, Stony Brook. Exter Software, Setanket, New York.

Sellwood, B. W., 1978. Shallow-Water Carbonate Environments: Sedimentary Environments and Facies: 10:259-313.

Shinn, E. A., 1983. Tidal Flat Environment: AAPG Memoir 33: 173-210.

Smith, L. B., and Read, J. F., 1999. Application of high-resolution sequence stratigraphy to tidally influenced Upper Mississippian carbonates, Illinois Basin: SEPM, Special Publication no. 63, p. 107-126. 
Smith, L. B., and Read, J. F., 2000. Rapid onset of late Paleozoic glaciation on Gondwana: Evidence from Upper Mississippian strata of the Midcontinent, United States: Geology, v. 28, no. 3, p. 279-282.

Smith, L. B., and Read, J. F., 2001. Discrimination of local and global effects on Upper Mississippian stratigraphy, Illinois Basin, USA: Journal of Sedimentary Research, v. 17, no. 6, p. 985-1002.

Tissue, E. C., 1986. Paleoecology and paleoenvironments of the Upper Greenbrier - Lower Mauch Chunk Transition, Garrett County, Maryland: West Virginia University, Masters Thesis, $214 \mathrm{p}$.

Whisonant, R. C., and Scolaro, R. J., 1980. Tide-Dominated Coastal Environments in the Mississippian Bluefield Formatin of Eastern West Virginia: Proceedings of the Fifth Symposium on Coastal Sedimentology, v.3, p. 593-636.

Wilson, J. L., 1975. Carbonate Facies in Geologic History, p. 20-29, 348-360 and 369-375.

Wilson, J. L., and Jordan, C., 1983. Middle Shelf: AAPG Memoir 33:297-344.

Wray, L. L., and Smosna R. A., 1982. Sedimentology of a carbonate red-bed association, Mississippian Greenbrier group, eastern West Virginia: Southeastern Geology, v. 23, no. 2, p. 99 108.

Yang, C., 1998. Basin Analysis of the Carboniferous Strata in Central and Southern West Virginia Using Sequence Stratigraphic Principles: West Virginia University, PhD Dissertation, 313 p. 


\section{APPENDIX 1}

Detailed Stratigraphic Columns and Field Notes 


\section{Hopwood}

Unit 1

$1.7 \mathrm{~m}$ from top of lower limestone bed (Greenbrier).

Calcareous mudstone.

Mottled purple - grey in places.

No sedimentary structures.

No fossils.

Sharp contact with the overlying unit.

Sample(s): HW-1.

Unit 2

$0.55 \mathrm{~m}$.

Limestone (uppermost Greenbrier) - mottled argillaceous micritic limestone.

Color: fresh - light grey mottled purple; weathers - tan.

Sedimentary structures: laminae present but not dominant.

Fossils: unidentifiable recrystalized fossil fragments found.

Sample(s): HW-2 *.

Unit 3

$1.85 \mathrm{~m}$.

Calcareous Mudstone.

Red throughout.

No sedimentary structures.

Fossils found but not throughout: ostracodes and brachiopods.

Comments: top of this unit is marked by $10 \mathrm{~cm}$ of green mudstone.

Sample(s): HW-3

Unit 4

$0.85 \mathrm{~m}$.

Mudstone.

Color: unit is mottled; consists of red, grey, and yellow mudstones.

Laminations are found in the lowermost yellow unit.

No fossils found.

Comments: this unit bears striking resemblance to the Saprolite of Charcoal Hill - Unit \#5.

Sample(s): HW-4a, HW-4b.

Unit 5

$0.30 \mathrm{~m}$.

Limestone - micritic, fairly clean.

Color: fresh - light grey; weathers - tan.

Hummocky cross-stratification is found in a more silty layer in the uppermost $4 \mathrm{~cm}$ of this limestone.

No fossils found.

Sample(s): HW-5 *. 


\section{Unit 6}

$4.7 \mathrm{~m}$ exposed remainder covered.

Mudstone.

Red throughout.

No sedimentary structures.

No fossils found.

Comments: 25-30 m of red mudstone above this unit around bend in road - not measured. This grades up into shales and sandstones.

Sample(s): HW-6. 


\section{Hopwood}

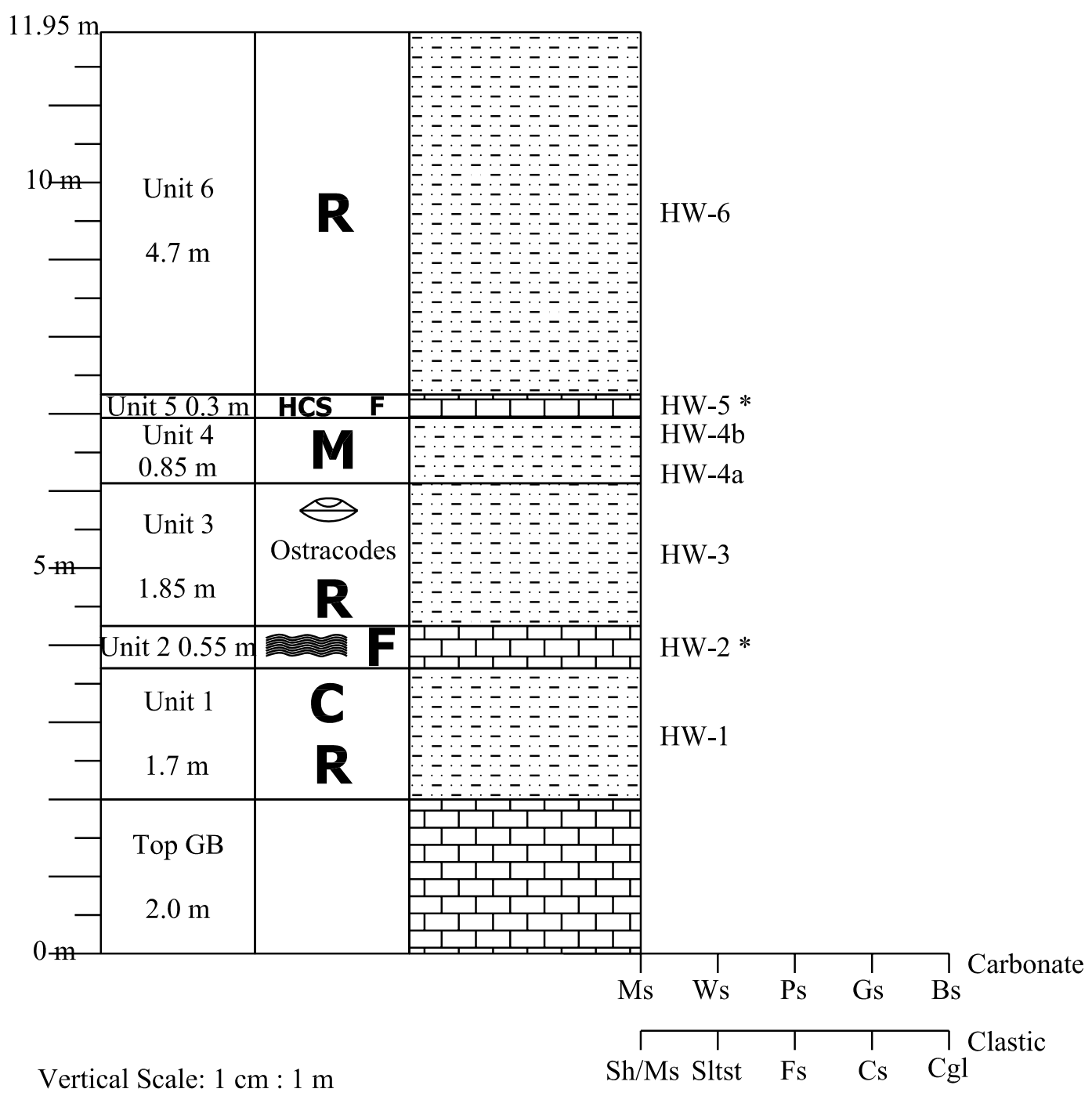




\section{Lake Lynn}

Unit 1

$1.0 \mathrm{~m}$.

Limestone (top of Greenbrier) - argillaceous micrite.

Color: fresh - grey; weathers - tan.

Sedimentary structures: laminae are present throughout, mudcracks evident on some bedding planes.

No fossils.

Sharp contact with overlying unit.

Sample(s): LL-1.

Unit 2

$0.32 \mathrm{~m}$.

Calcareous Siltstone.

Red / green mottled.

No sedimentary structures.

No fossils.

Comments: unit is separated from top of Greenbrier by a thin $(4 \mathrm{~cm})$ green shale.

Sample(s): LL-2.

\section{Unit 3}

$0.92 \mathrm{~m}$.

Mudstone.

Red / green mottled.

No sedimentary structures.

No fossils.

Sample(s): LL-3.

\section{Unit 4}

$0.52 \mathrm{~m}$.

Siltstone.

Red / green mottled.

No sedimentary structures.

No fossils.

Comments: mottling in this unit is calcareous for the green ONLY the red is not calcareous.

Overall this unit is very similar to unit 2 with much more green.

Sample(s): LL-4.

Unit 5

$2.58 \mathrm{~m}$.

Mudstone.

Red.

No sedimentary structures.

Fossils: all found are very small 2-3 mm; gastropods, bivalves, ?ostracodes? 
Comments: irregularly rounded calcareous nodules found throughout.

Sample(s): LL-5.

Unit 6

$1.0 \mathrm{~m}$.

Calcareous Mudstone.

Red.

No sedimentary structures.

No fossils.

Comments: lowest $.18 \mathrm{~m}$ of this unit is a calcareous, mottled red / green siltstone that grades up into the mudstone.

Sample(s): LL-6.

\section{Unit 7}

$0.1 \mathrm{~m}$.

Limestone - argillaceous wackestone, highly fossiliferous.

Color: fresh - grey; weathers - tan.

No sedimentary structures.

Fossils: throughout and abundant; brachiopods - Anthracospirifer, Diaphragmus, Eumetria, Orthotetes, other - orthocone, bryozoan.

Comments: lateral thickness varies as does how argillaceous the limestone becomes. These two variables appear independent of one another but complete fossils are easily extracted from the "softer" areas.

Sample(s): LL-7.

Unit 8

$4.5 \mathrm{~m}$.

Mudstone.

Grey.

No sedimentary structures.

Fossils: throughout, brachiopods - Anthracospirifer, Orthotetes, bivalves, complete fossils hard to find due to the lithology.

Comments: a small micritic limestone bed $(4 \mathrm{~cm})$ is located at $3.15 \mathrm{~m}$ (LL-8b). At top the unit grades into a tan mudstone.

Sample(s): LL-8a, LL-8b.

Unit 9

$0.65 \mathrm{~m}$.

Limestone - a very argillaceous wackestone grading up into a more clean wackestone (above unit).

Color: fresh - grey; weathers - brown.

No sedimentary structures.

Fossils: throughout and abundant; brachiopods -Anthracospirifer, Composita, Diaphragmus, gastropods - Straparolus, Bellerphon, bivalves - Wilkingia, ?pecten?, other - crinoid debris, encrusting bryozoan. 
Comments: this unit has more resistant limestones interbedded with less resistant more argillaceous limestones both approximately $8 \mathrm{~cm}$.

Sample(s): LL-9 *.

Unit 10

$3.15 \mathrm{~m}$.

Limestone - wackestone.

Color: fresh - grey; weathers - tan.

Few thin $(4 \mathrm{~cm})$ laminated beds between thicker $(10 \mathrm{~cm})$ beds.

Fossils: abundant throughout; brachiopods - Anthracospirifer, Composita, Diaphragmus, Orthotetes, ?Productus?, bivalves - Sanginolites, Wilkingia, gastropods - Bellerophon, Straparolus, some unidentified, other - crinoid debris.

Sample(s): LL-10 *. 


\section{Lake Lynn}

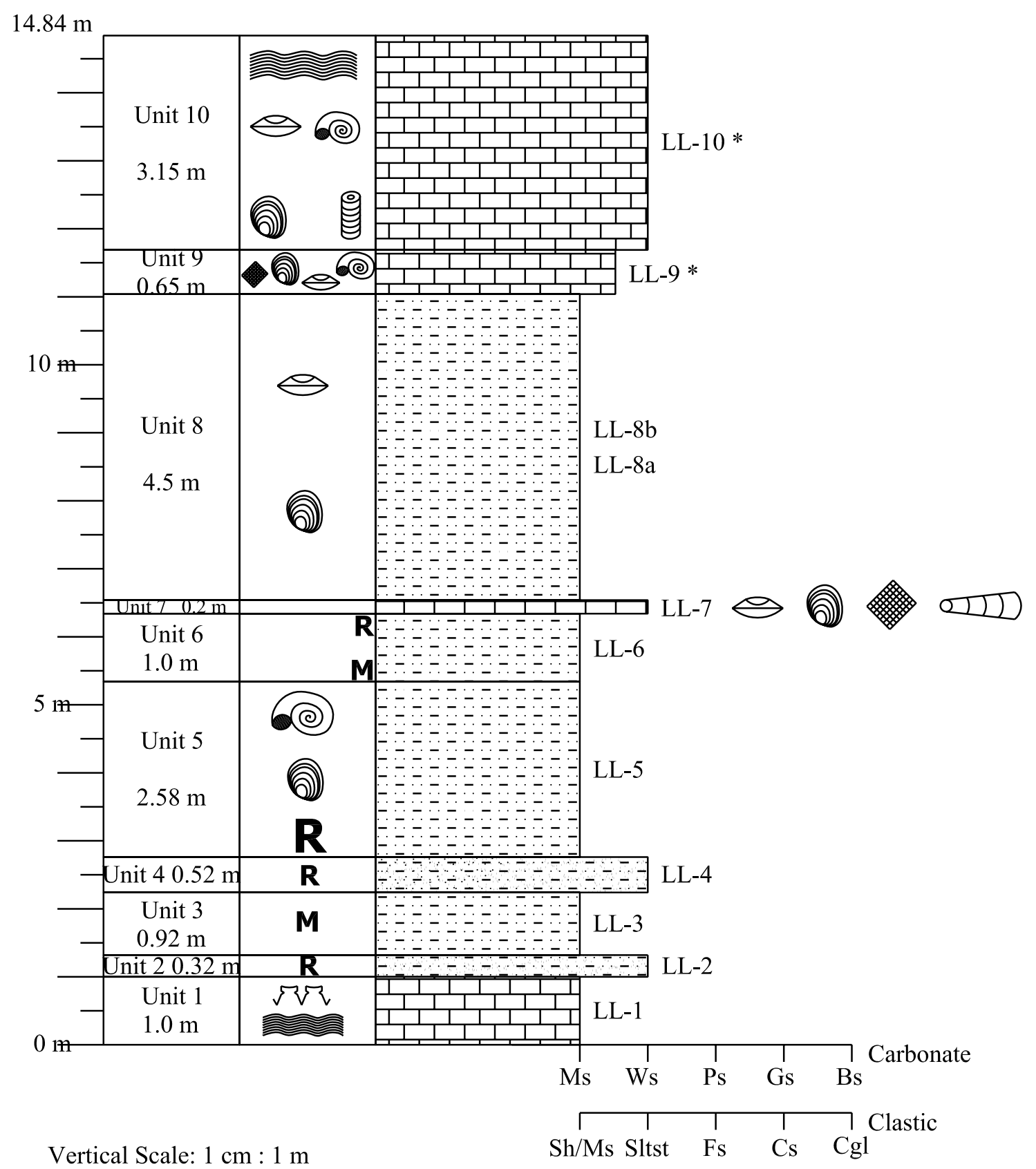




\section{Charcoal Hill}

\section{Unit 1}

Top 2 m only. Limestone (Greenbrier).

Argillaceous micritic limestone - shows diffuse bedding (bioturbated).

Color: fresh is medium grey weathered is dusky red.

Sedimentary structures: Shale clasts are found on weathered surfaces ( $1 \mathrm{~cm} \mathrm{x} 2 \mathrm{~mm}$ red).

Fossils: some fossil grains present but unidentifiable on fresh surfaces and only crinoid stems are recognizable on weathered surfaces.

Sharp contact with overlying unit.

Sample(s): CH-1a, CH-1b.

\section{Unit 2}

$1.92 \mathrm{~m}$.

Silty Mudstone.

Dusky red.

Not fissile - is definitely a mudstone.

Sedimentary structures: no laminations - likely bioturbated from plant roots.

No fossils.

Comments: Top of unit is marked by $8 \mathrm{~cm}$ of green mudstone.

Sample(s): CH-2a, CH-2b, CH-2c.

Unit 3

$2.05 \mathrm{~m}$.

Calcareous interbedded thin siltstones and silty shales - alternating every half-meter.

Red throughout - green at basal $16 \mathrm{~cm}$.

Shales are fissile, siltstones are thin bedded.

No sedimentary structures.

Fossils: brachiopods - Anthracospirifer, Orbiculoides, bivalves - Aviculopecten, Pecten, burrowing bivalves, epifaunal bivalves, other - crinoid cup.

Sample(s): CH-3a, CH-3b.

Unit 4

$0.95 \mathrm{~m}$.

Silty Mudstone - same as unit 2.

Dusky red.

Not fissile - is definitely a mudstone.

Sedimentary structures: no laminations - likely bioturbated from plant roots.

No fossils.

Sample(s): CH-4 
Unit 5

$1.8 \mathrm{~m}$.

Clay-mudstone.

Much lighter in color than surrounding units - ochre, grey, mottled organic, dark brown, ash grey.

Layers are distinct but contacts are gradational.

No sedimentary structures.

Fossils: brachiopods - Anthracospirifer, Composita, Diaphragmus, Orbiculoides, Orthotetes, bivalves - burrowing clam, other - crinoid columnals.

Comments: It was originally believed this unit was a paleosol based on the colors and organization of the unit. Several fossils found in the unit have changed this interpretation to that of a saprolitic limestone (Reynolds?). Different colors found throughout this unit reflect the varying amounts of leaching the original limestone has undergone.

Sample(s): CH-5a, CH-5b, CH-5c.

Unit 6

$2.7 \mathrm{~m}$ exposed, remainder covered.

Mudstone.

Red throughout, darker at top.

No sedimentary structures.

No fossils.

Sample(s): CH-6 


\section{Charcoal Hill}

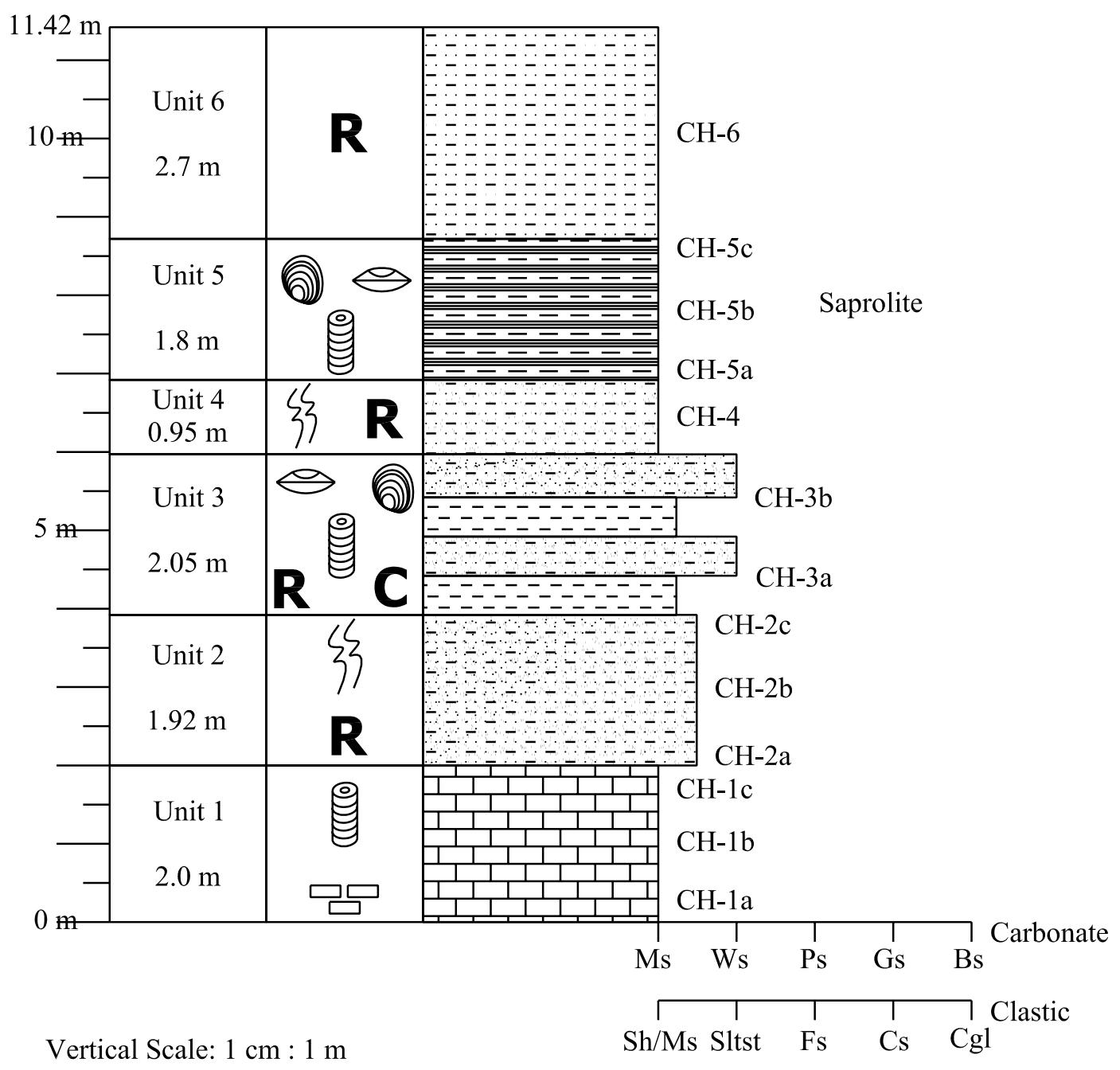




\section{Scherr}

Unit 1

$2.0 \mathrm{~m}$.

Limestone (top of Greenbrier) - argillaceous micrite / fossiliferous wackestone

Color: fresh - grey; weathers - tan.

Sedimentary structures: laminae present - but sporadic.

Fossils: mainly brachiopods - Composita, Diaphragmus, Orthotetes, other - crinoid columnals.

Sharp contact with overlying unit.

Sample(s): SH-1a,b,c.

Unit 2

$2.9 \mathrm{~m}$.

Calcareous Claystone.

Red.

No sedimentary structures.

No fossils.

Sample(s): SH-2.

Unit 3

$2.75 \mathrm{~m}$.

Calcareous Mudstone.

Red.

No sedimentary structures.

No fossils.

Comments: calcareous nodules throughout in upper half of this unit.

Sample(s): SH-3.

Unit 4

$0.75 \mathrm{~m}$.

Calcareous Silty Claystone.

Olive grey.

No sedimentary structures.

No fossils.

Sample(s): SH-4.

Unit 5

$0.65 \mathrm{~m}$.

Shale.

Tan grey.

This unit is very fissile.

No sedimentary structures.

Fossils: present and very small hard to identify - bits of ?brachs? and ?bivalves?

Comments: samples need to be identified under a scope in the lab.

Sample(s): SH-5, SH-5grab. 
Unit 6

$3.45 \mathrm{~m}$.

Limestone - argillaceous micrite / fossiliferous wackestone.

Color: fresh - dark grey; weathers - tan.

No sedimentary structures.

Fossils: sporadic - some recrystallized - brachiopods - Anthracospirifer, Lingula? gastropods Straparolus, other - crinoid columnals, in-filled burrows: no complete body fossils.

Sample(s): SH-6a *,b *.

\section{Unit 7}

$1.95 \mathrm{~m}$.

Sandstone - fine grained and micaceous.

Red.

No sedimentary structures.

No fossils.

Sample(s): SH-7.

\section{Unit 8}

$2.0 \mathrm{~m}$ exposed everything up slope covered.

Mudstone.

Red.

No sedimentary structures.

No fossils.

Sample(s): SH-8. 


\section{Scherr}

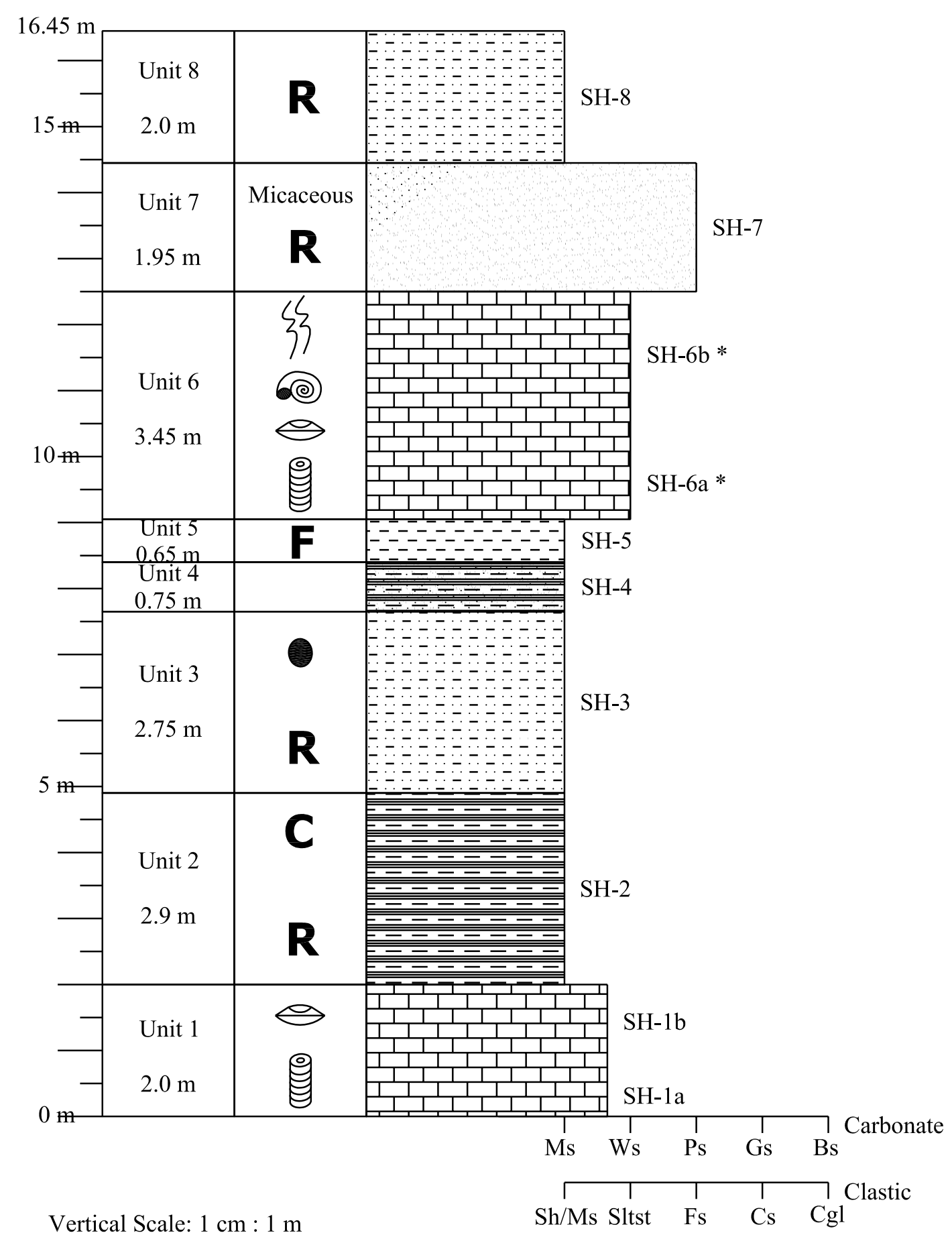




\section{Elkins}

Unit 1

$+2.0 \mathrm{~m}$.

Limestone (top of Greenbrier) - micrite.

Color: fresh - medium grey; weathers - light grey.

No sedimentary structures.

Fossils: dominantly fossil hash - few individuals found: crinoid and brachiopod bits,

Diaphragmus, Orthotetes.

Sharp contact with overlying unit.

Sample(s): EK-1.

Unit 2

$1.35 \mathrm{~m}$.

Shale.

Color: blue-grey.

Sedimentary structures: runzelmarks found on top of small sandstone bed.

Fossils: very sparse and hard to identify - crinoid columnals.

Sample(s): EK-2.

Unit 3

$2.25 \mathrm{~m}$.

Sandstone - very fine to fine grained.

Color: fresh - dark green; weathers - dull green.

No sedimentary structures.

No fossils.

Comments: gradational contact with lower unit, chert nodules present but rare.

Sample(s): EK-3.

Unit 4

$1.0-3.0 \mathrm{~m}$.

Calcareous silty mudstone.

Color: mottled red - green.

No sedimentary structures.

No fossils.

Comments: can see channel structures in unit above causing lateral thickness variation. Sample(s): EK-4.

\section{Unit 5}

$0.5-2.0 \mathrm{~m}$.

Sandstone - medium to fine grained - micaceous.

Color: light grey.

Sedimentary structures: ripple beds $+/-3 \mathrm{~cm}$, few small-scale cross beds.

No fossils.

Comments: can see definite channel structures cutting into lower unit, lower parts of channels look massively bedded. 
Sample(s): EK-5.

Unit 6

$0.95 \mathrm{~m}$.

Calcareous silty mudstone.

Color: two distinct layers: upper $0.2 \mathrm{~m}$ green; lower $0.75 \mathrm{~m}$ red.

No sedimentary structures.

Fossils: small and poorly preserved only found in uppermost layer; clams, ?Lingula?.

Sample(s): EK-6.

\section{Unit 7}

$4.9 \mathrm{~m}$.

Interbedded sandstones and shales.

Color: medium grey.

Sedimentary structures: small-scale cross beds.

Fossils: lower part of section - clams, Wilkingia, Orthotetes: upper limestone channel

(biomicrite) - fossils abundant; Anthracospirifer, Diaphragmus, Orthotetes.

Comments: unit is capped by a limestone channel fill - pinches out laterally.

Sample(s): EK-7.

Unit 8

$1.3 \mathrm{~m}$.

Calcareous shale.

Color: dark grey/green.

No sedimentary structures.

Fossils: dominantly brachiopods; Diaphragmus, Orthotetes, also found; Edmondia, crinoid columnals, clams, gastropods.

Comments: this unit has a gradational contact with the overlying unit 9 .

Sample(s): EK-8.

Unit 9

$5.35 \mathrm{~m}$.

Limestone - biomicrite.

Color: fresh - dark grey; weathers - medium grey.

No sedimentary structures.

Fossils: brachiopod hash, Anthracospirifer, Composita, Diaphragmus, Orthotetes, crinoid columnals, gastropod.

Comments: shaly interbeds at base massive at top.

Sample(s): EK-9.

Unit 10

$1.4 \mathrm{~m}$.

Shale.

Color: dark grey.

Sedimentary structures: ?slickensides?

No fossils. 
Samlpe(s): EK-10.

Unit 11

$0.7 \mathrm{~m}$.

Limestone - wackestone.

Color: fresh - dark grey; weathers - medium grey.

No sedimentary structures.

Fossils: Anthracospirifer, Diaphragmus, Orthotetes, crinoid columnals, bryozoans.

Sample(s): EK-11.

Unit 12

$2.6 \mathrm{~m}$.

Shale.

Color: grey.

No sedimentary structures.

No fossils.

Sample(s): EK-12

Unit 13

$+2.0 \mathrm{~m}$

Shale.

Color: base - blue-grey grades into top - red / green.

No sedimentary structures.

?Fossils?

Comments: measuring from bucket truck - could not reach higher to get samples or look for fossils.

Sample(s): none. 


\section{Elkins}

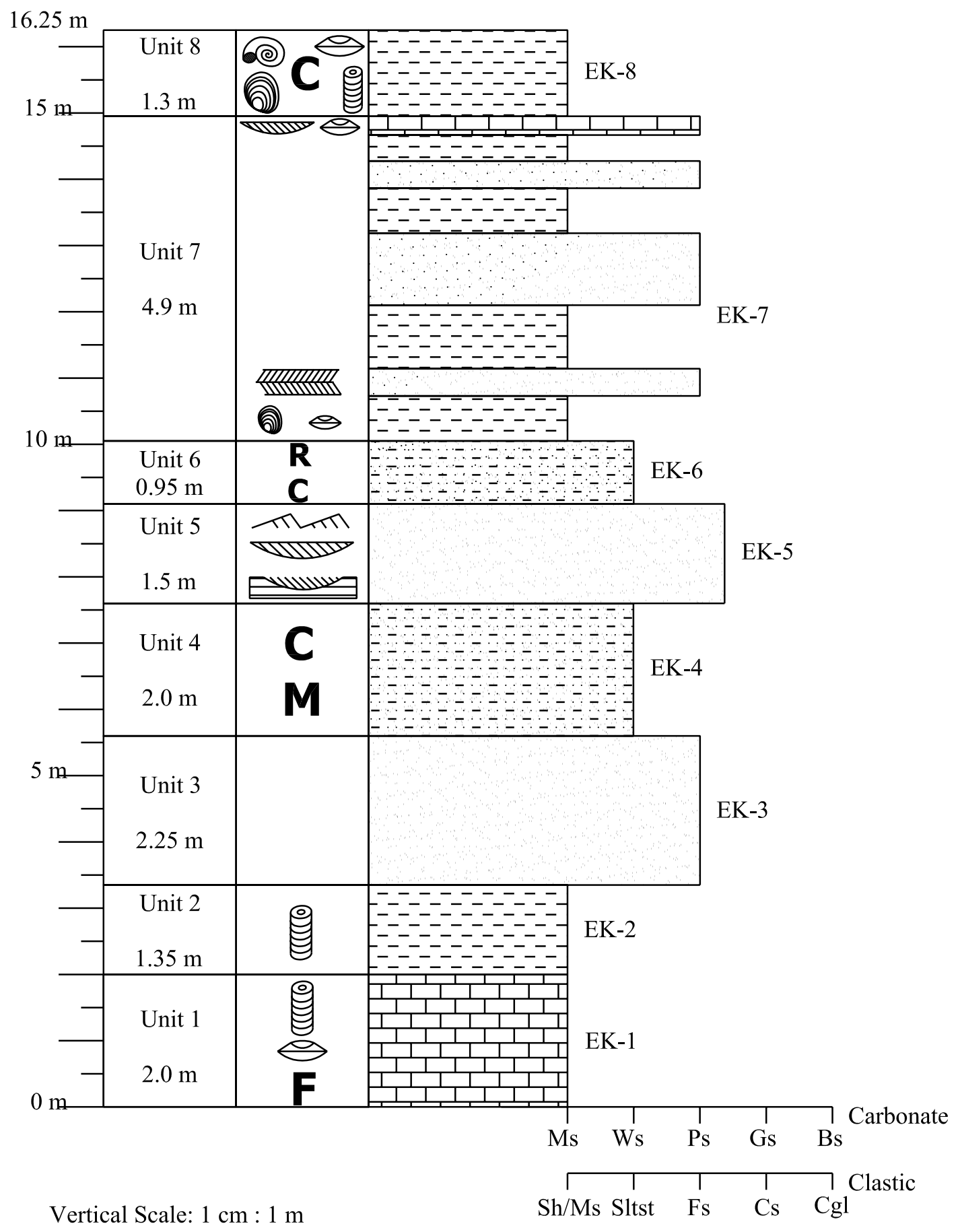




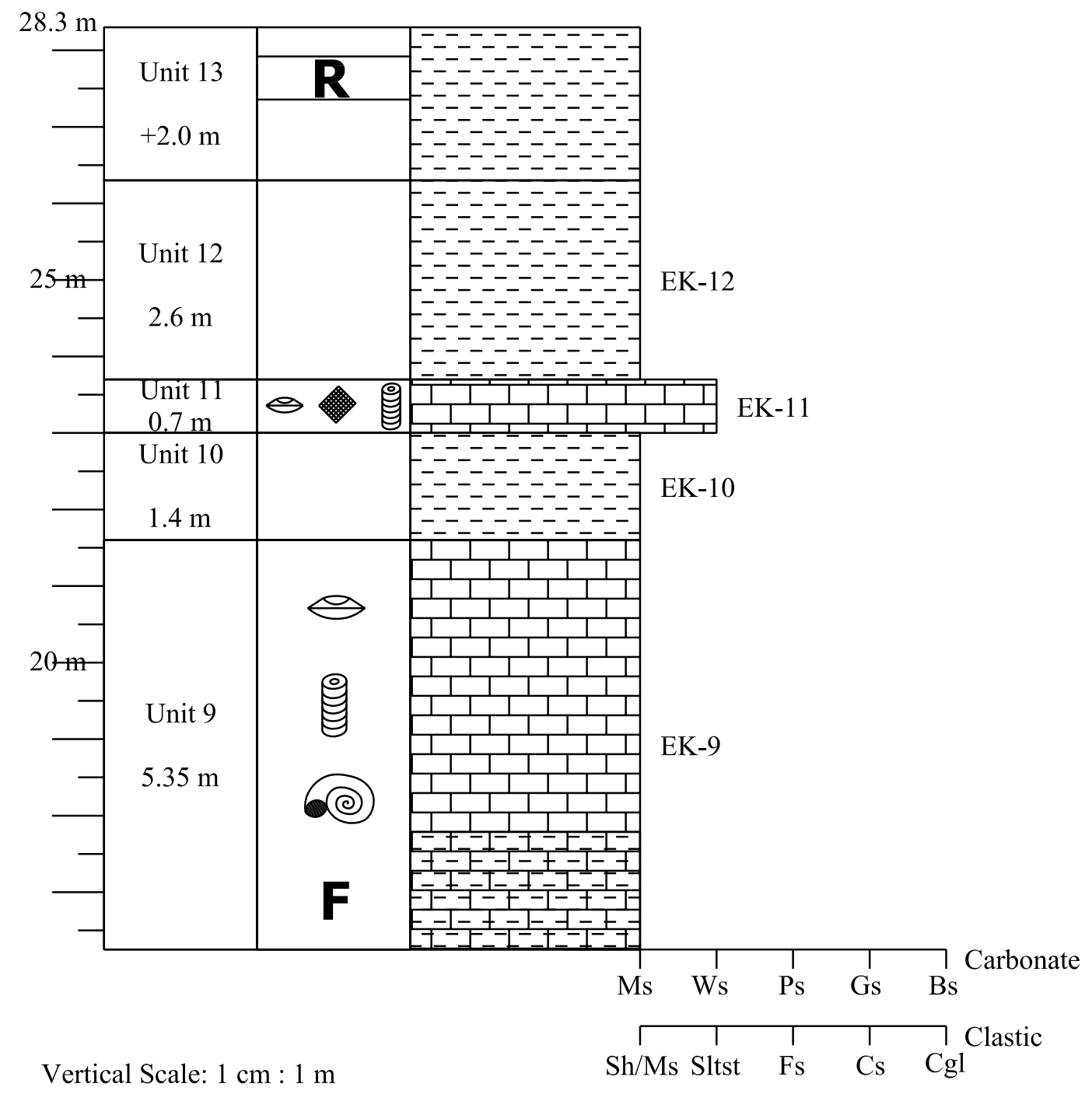




\section{Edray}

Unit 1

$1.0 \mathrm{~m}$.

Limestone (top of Greenbrier) - ooid grainstone.

Color: fresh - medium grey; weathers - tan grey.

Sedimentary structures: cross bed sets.

Fossils: mainly fossil hash - brachs and bivalves.

Comments: poorly exposed in a wash along a hillside in a grown-up field, nearest major

landmark is a small group of large oak trees.

Sharp contact with overlying unit.

Sample(s): ER-1.

Unit 2

$93.25 \mathrm{~m}$.

Shale with interbedded sandstones.

Color: varies - but dominantly red / brown.

Sedimentary structures: ripple scale cross beds and soft sediment deformation in sandstones.

Fossils: no body fossils only trace fossils.

Comments: this unit was measured primarily to gauge and compare thickness of the different sections - detailed descriptions of this unit are outside the scope of this project.

*All measurements are from top Greenbrier to base of unit.

\section{Unit 2a}

$4.80 \mathrm{~m}$ above Greenbrier.

$0.5 \mathrm{~m}$.

Limestone (mudstone).

Color: fresh - grey: weathers - tan.

No fossils.

No sedimentary structures.

Unit $2 b$

$30.80 \mathrm{~m}$ above Greenbrier.

$0.5 \mathrm{~m}$.

Ooid grainstone.

Color: fresh - grey: weathers - tan.

No fossils.

No sedimentary structures.

Unit 2c

$46.90 \mathrm{~m}$ above Greenbrier.

$2.85 \mathrm{~m}$.

Ooid grainstone.

Color: fresh - grey: weathers - tan. 
No fossils.

No sedimentary structures.

Unit $2 \mathrm{~d}$

$71.0 \mathrm{~m}$ above Greenbrier.

$1.40 \mathrm{~m}$.

Fine-grained quartz sandstone.

Tan on both fresh and weathered surfaces.

Fossils - Anthracospirifer, Diaphragmus, pelmatozoan debris, burrows.

Sedimentary structures - small-scale ripples, trough cross-beds.

Sample(s): none.

Unit 3

$2.52 \mathrm{~m}$.

Limestone $-1.67 \mathrm{~m}$. heavily bioturbated argillaceous wackestone grades into $0.85 \mathrm{~m}$.

argillaceous fossiliferous wackestone.

Color: fresh - grey; weathers - light grey.

Sedimentary structures: laminae in upper $0.85 \mathrm{~m}$.

Fossils: brachiopods - Composita, Diaphragmus, Orthotetes, bivalves - Wilkingia, gastropods Straparolus.

Sample(s): ER-3.

\section{Unit 4}

$2.6 \mathrm{~m}$.

Limestone - lower $1.73 \mathrm{~m}$. fossiliferous calc-shale wackestone, upper $0.87 \mathrm{~m}$. interbedded wackestones and shaly limestones.

Color: fresh - dark grey; weathers - very dark grey.

No sedimentary structures.

Fossils: dominated by brachiopods - Diaphragmus, Orthotetes, Productus (few).

Comments: wackestones increase in thickness and coarseness upward.

Sample(s): ER-4a,b,c *.

Unit 5

$2.7 \mathrm{~m}$.

Limestone - fossiliferous wackestone.

Color: fresh - grey; weathers - brown tan.

Sedimentary structures: weakly hummocked beds and some cross bedding throughout.

Fossils: brachiopods - Anthracospirifer, Diaphragmus dominant, Orthotetes.

Sample(s): ER-5 *. 
Unit 6

$3.57 \mathrm{~m}$.

Interbedded calcareous shales and fossiliferous micrites.

Color: shales - grey both fresh and weathered; limestones - fresh - grey; weathered - tan.

Sedimentary structures: all micrite beds appear to be channel fills, small scale cross beds, ripples on tops of some beds.

Fossils: highly fossiliferous - brachiopods - Anthracospirifer, Diaphragmus, Orthotetes,

Productus.

Sample(s): ER-6 *.

Unit 7

$2.0 \mathrm{~m}$.

Limestone - argillaceous fossiliferous micrite / wackestone.

Color: fresh - brown grey; weathers - grey brown.

Sedimentary structures: irregular top and bottom surfaces.

Fossils: few and far between - unit bioturbated ? brachiopods - Anthracospirifer, Composita.

Sample(s): ER-7 *.

Unit 8

$2.58 \mathrm{~m}$.

Calcareous Shale.

Color - dark grey both fresh and weathered.

No sedimentary structures.

Fossils: poorly fossiliferous - unidentifiable bits due to fissility of shale.

Comments: sparsely fossiliferous micrite bed $0.25 \mathrm{~m}$ near bottom of unit. Max flooding surface possibly in this unit.

Sample(s): ER-8.

Unit 9

$2.65 \mathrm{~m}$.

Interbedded Limestones and Shales.

Color: shale - dark grey both fresh and weathered; limestones - fresh - grey; weathers - tan.

No sedimentary structures.

Fossils: both fossiliferous, dominantly brachiopods.

Sample(s): ER-9a *,b,c,d *,e,f *.

Unit 10

$0.30 \mathrm{~m}$.

Interbedded Limestones and Shales.

Color: shale - dark grey both fresh and weathered; limestones - fresh - grey; weathers - tan.

No sedimentary structures.

Fossils: shales are barren, Archimedes found in limestone.

Samlpe(s): ER-10. 


\section{Unit 11}

$0.85 \mathrm{~m}$.

Calcareous Shale.

Color: dark grey both fresh and weathered.

No sedimentary structures.

Fossils: poorly fossiliferous - trilobite, brachiopod parts, Nuculid clam, fossil hash.

Sample(s): ER-11.

\section{Unit 12}

$1.39 \mathrm{~m}$.

Shale.

Olive tan.

Sedimentary structures: calcareous nodules.

Fossils: mildly fossiliferous throughout with higher concentrations around concretions, bivalves dominant, brachiopods, trilobite bits.

Sample(s): ER-12.

Unit 13

$2.81 \mathrm{~m}$.

Shale.

Olive tan.

No sedimentary structures.

No fossils.

Comments: unit capped by a small fine-grained, ripple cross bedded, quartz sandstone. Sample(s): ER-13. 


\section{Edray}

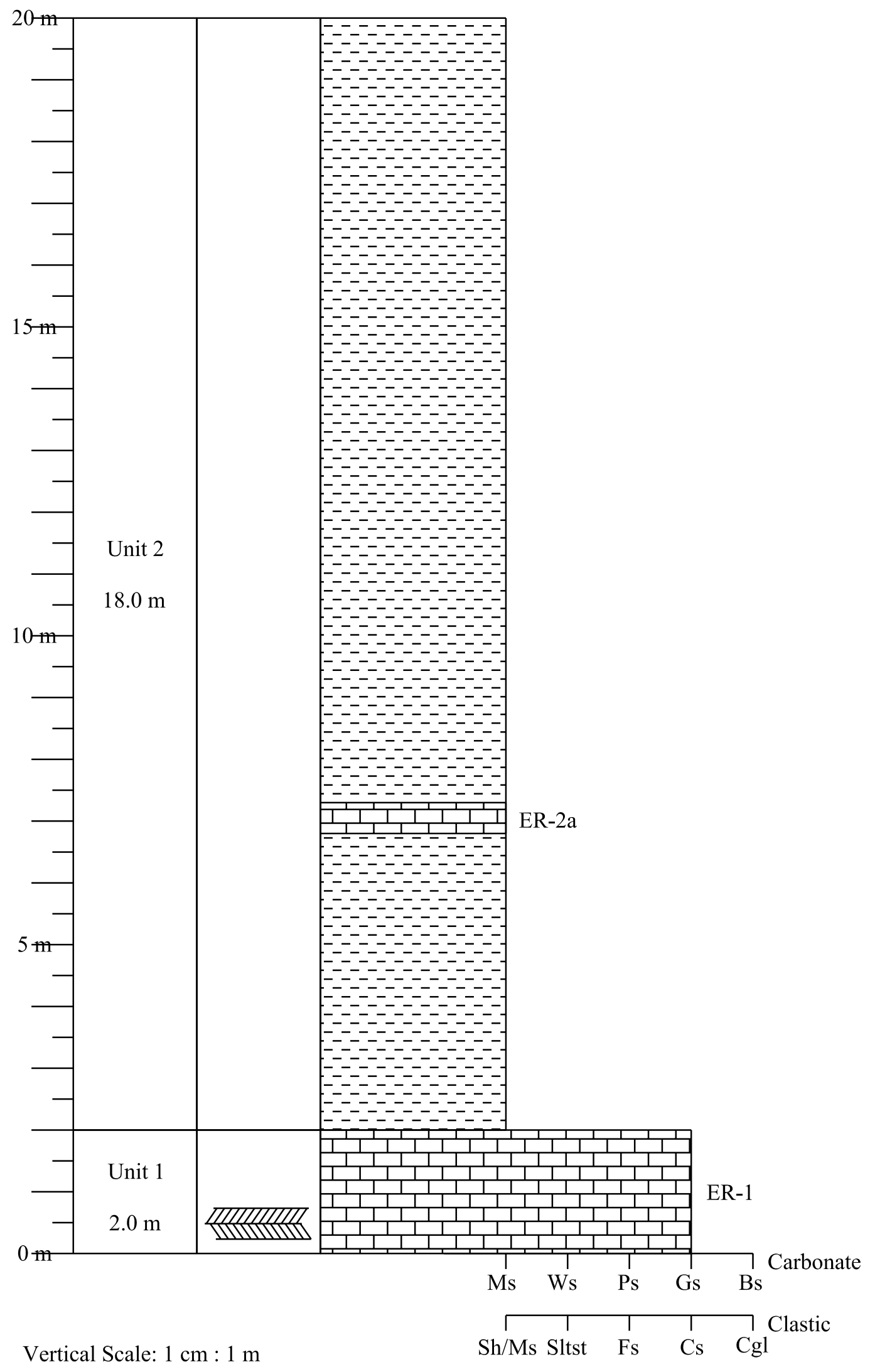




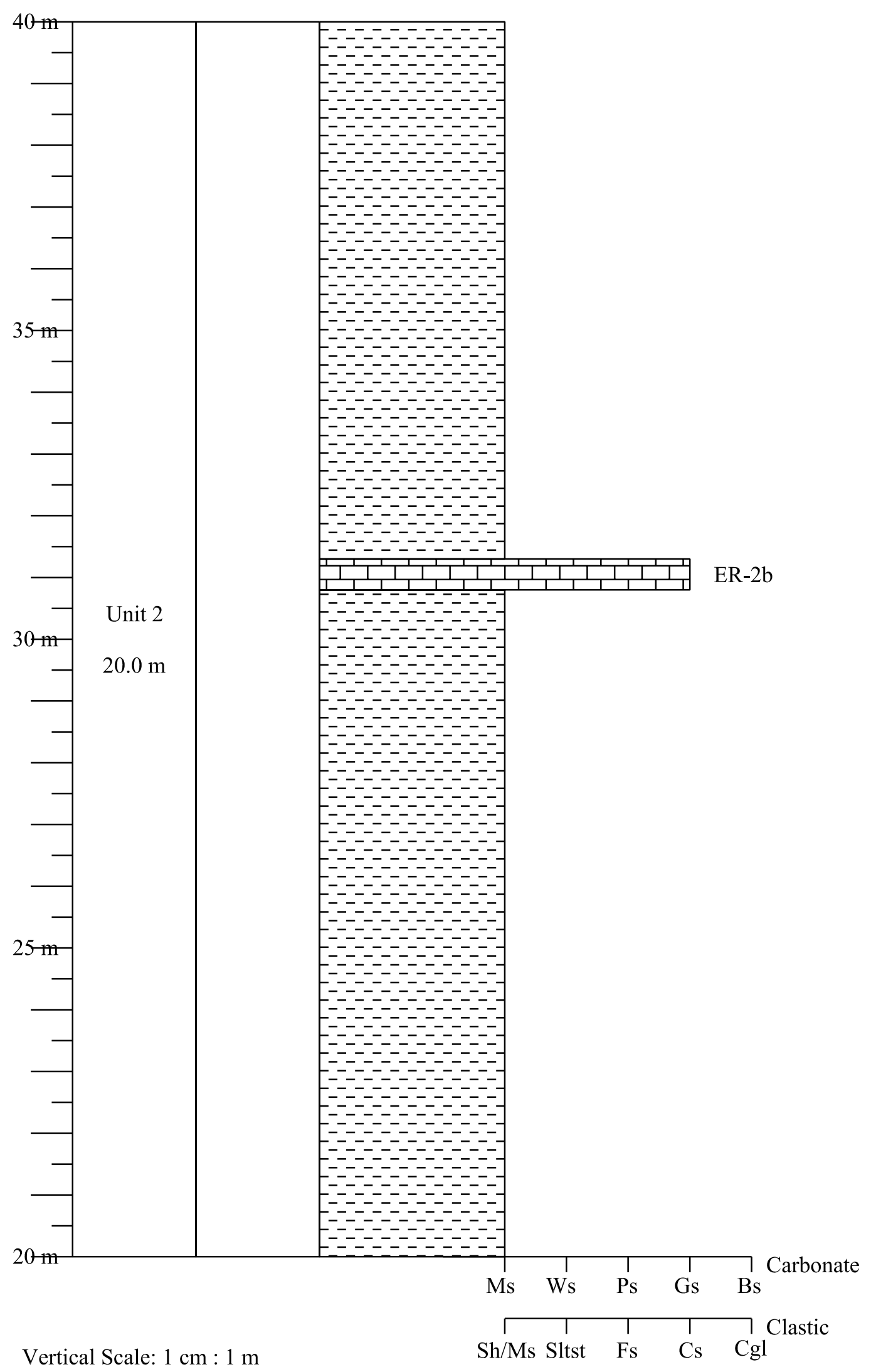




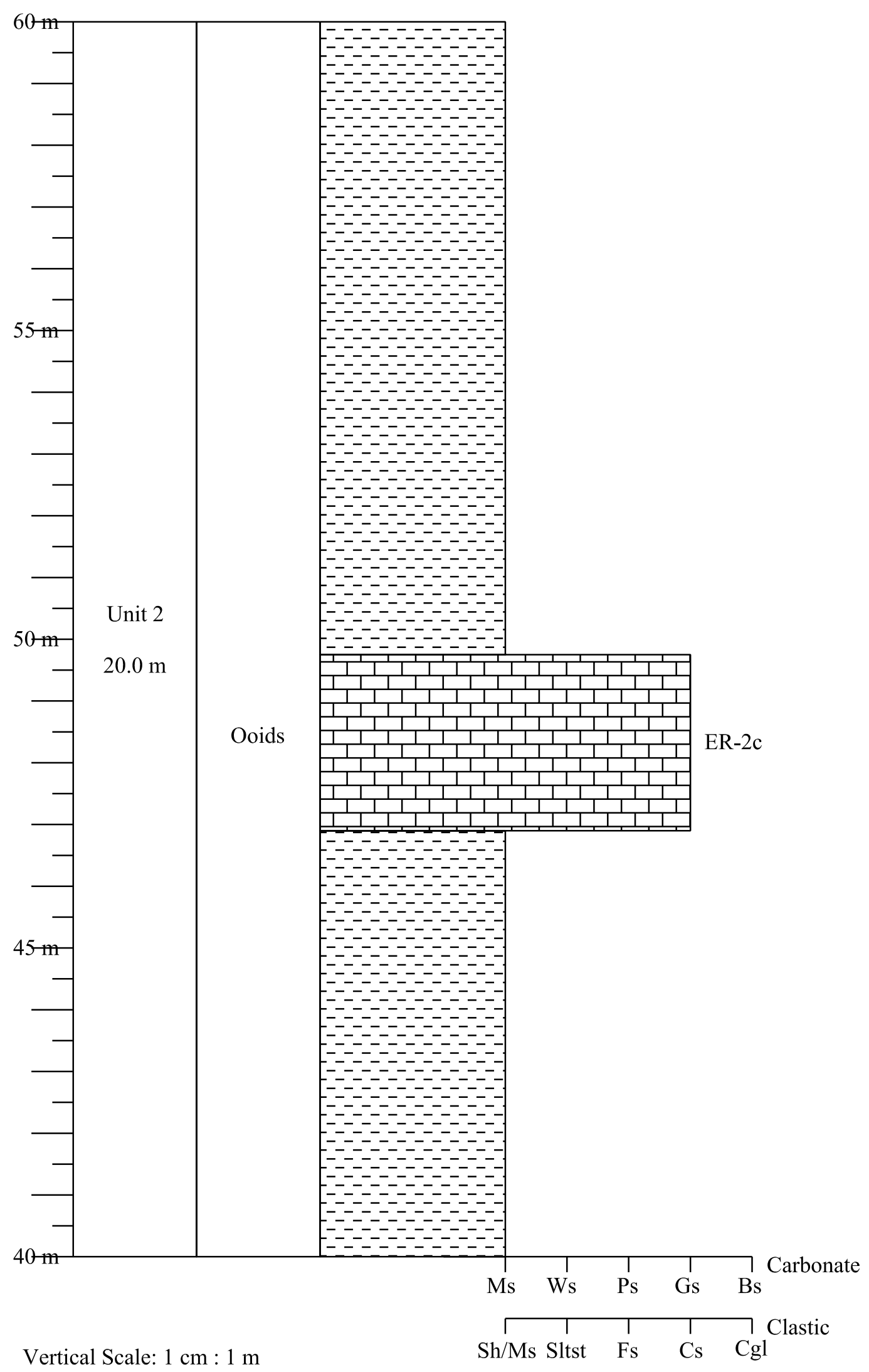




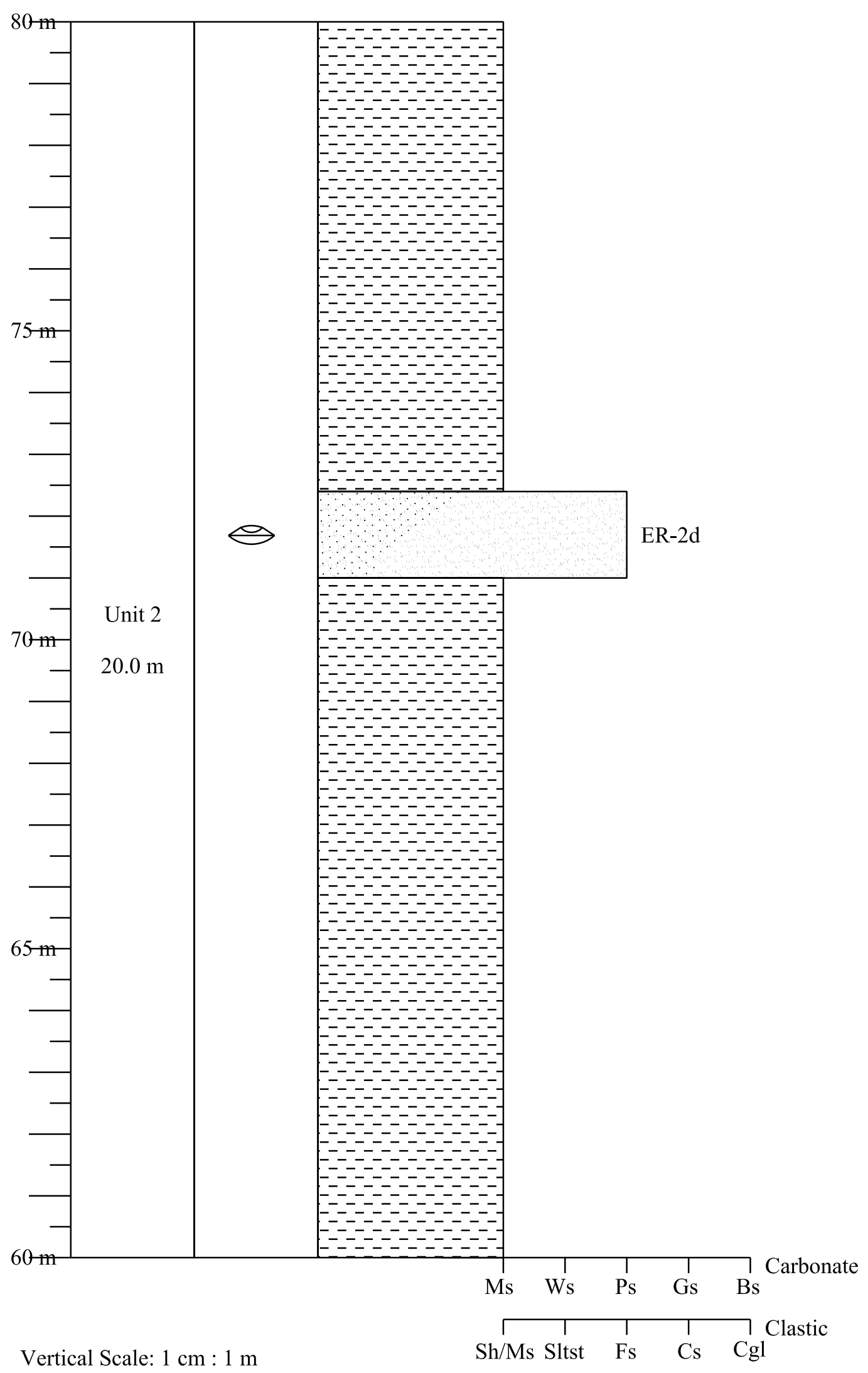




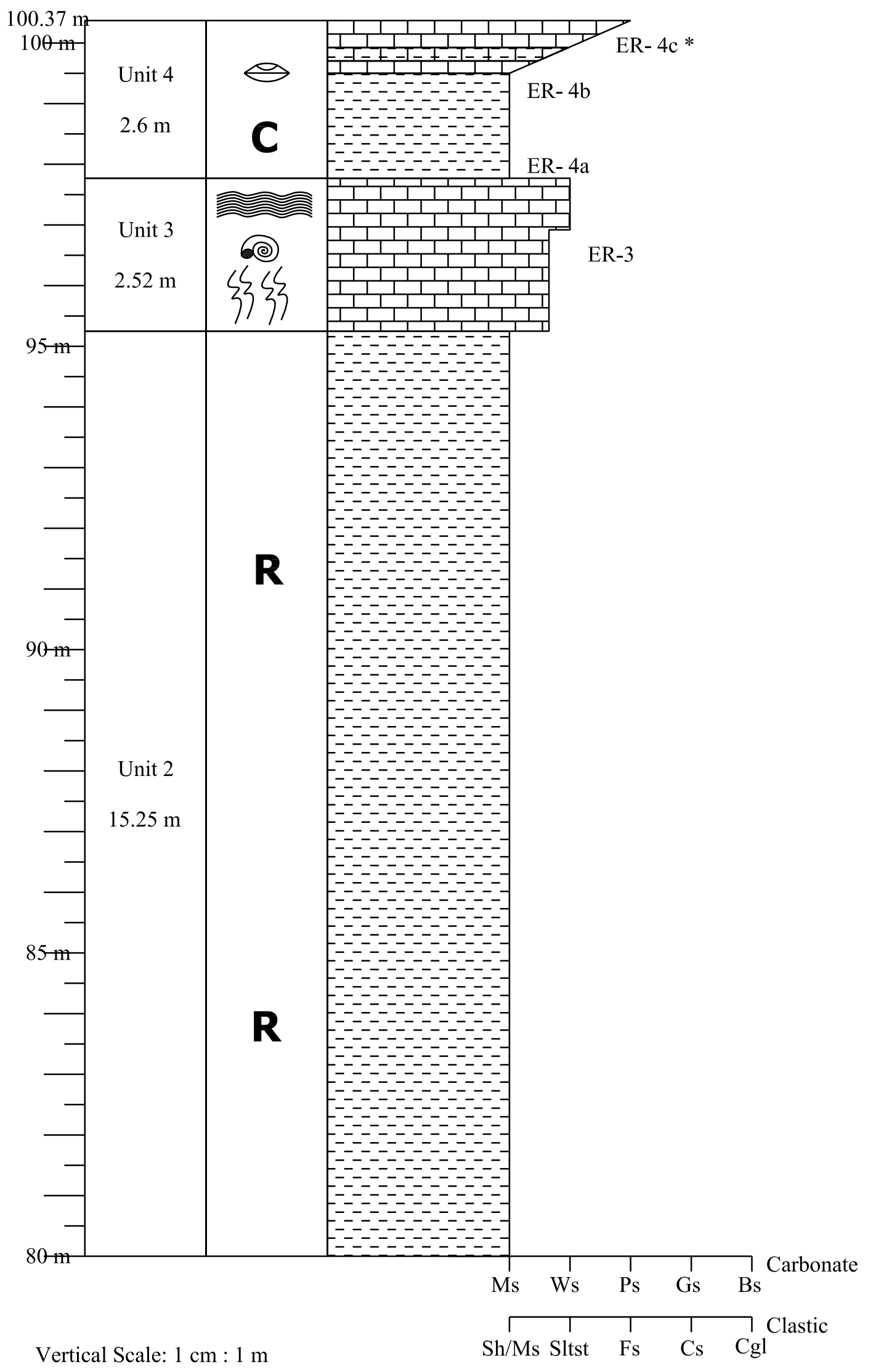




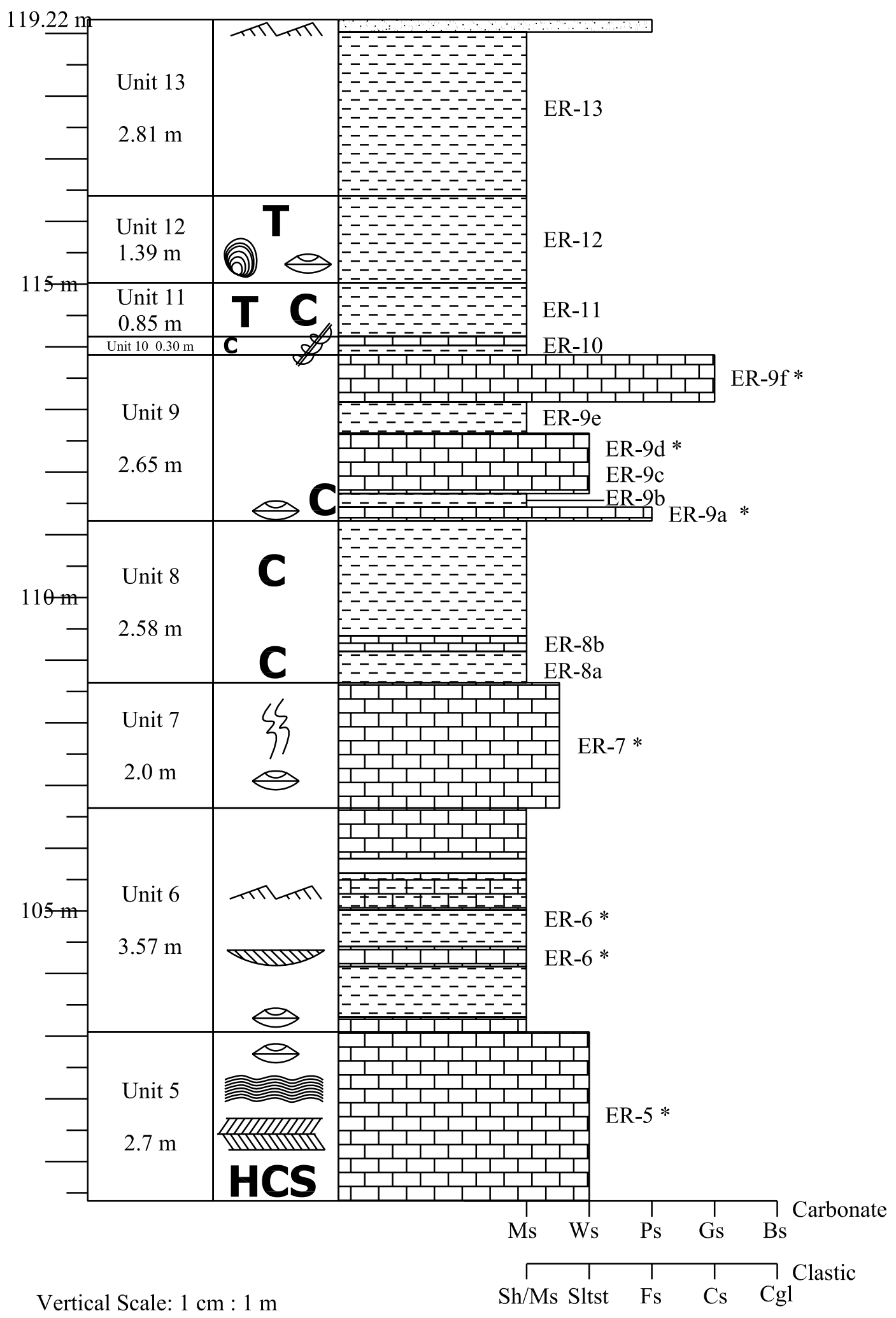




\section{Droop Mountain}

Unit 1

$2.0 \mathrm{~m}$.

Limestone (top of Greenbrier) -ooid grainstone / biomicritic wackestone.

Color: fresh - dark grey; weathers - tan.

Highly crossbedded throughout.

Fossils: brachiopods - Composita, gastropods - Straparolus, other - bryozoans, crinoid

columnals, carbonate grains (some recrystallized).

Sharp contact with overlying unit.

Sample(s): DM-1.

Unit 2

$64.0 \mathrm{~m}$.

Shale.

Color: varies but dominantly red/brown.

Shales are fissile few interbedded sandstones (10 cm max.)

Sedimentary structures: soft sediment deformation where sandstones overlie shales, ripples in both, interference ripples in some sands.

Fossils: unit has yielded no body fossils but trace fossils are common in both, a Rusophycus was found in the lower part of this unit.

Comments: this unit was measured primarily to gauge and compare thickness of the different sections - detailed descriptions of this unit are outside the scope of this project.

Sample(s): none.

\section{Unit 3}

$0.70 \mathrm{~m}$.

Calcareous Silty Mudstone.

Red.

No sedimentary structures.

No fossils.

Sample(s): none.

\section{Unit 4}

$0.75 \mathrm{~m}$.

Sandstone - very fine grained.

Olive tan.

No sedimentary structures.

Fossils: plant imprints.

Sampe(s): none.

Unit 5

$2.25 \mathrm{~m}$.

Calcareous Mudstone.

Grey blue.

No sedimentary structures. 
No fossils.

Comments: some thin interbedded fine grained sandstones, sporadic micritic concretions.

Sample(s): DM-5.

Unit 6

$2.65 \mathrm{~m}$.

Sandstone - varies from medium to very fine grained.

Olive tan.

Ripples evident in some beds, small scale scour bases.

Fossils: some fossil hash throughout - sparse brachiopod bits, some bioturbation.

Sample(s): DM-6.

\section{Unit 7}

$4.9 \mathrm{~m}$.

Limestone - clean micrite.

Color: fresh - medium grey; weathers - tan brown.

Massively bedded.

Sedimentary structures: entire unit has faint ripple scale cross beds, laminations are present but mainly in upper part of unit, some laminations are a very fine grained rippled sandstone.

Fossils: brachiopods - Anthracospirifer, Composita, Diaphragmus, Orthotetes, other - crinoid columnals.

Comments: chert nodules are found throughout this lower section, silicified brachiopods are common - typically found on the tops of the sandy laminations.

Sample(s): DM-7 *.

\section{Unit 8}

$1.09 \mathrm{~m}$.

Calcareous Siltstone.

Color: fresh - light grey; weathers - tan.

No sedimentary structures.

No fossils.

Comments: the uppermost $0.36 \mathrm{~m}$ of this unit is a highly argillaceous micritic limestone it is yellow brown both fresh and weathered possibly a chalk? This would be a good unit to have a thin section made of.

Sample(s): DM-8a,b *.

\section{Unit 9}

$1.72 \mathrm{~m}$.

Calcareous Mudstone.

Red.

No sedimentary structures.

No fossils.

Sample(s): DM-9. 
Unit 10

$3.32 \mathrm{~m}$.

Sandstone.

Purple red.

This unit is a series of interbedded medium to very fine grained sandstones.

Sedimentary structures: ripple-scale cross beds, small scale scour bases on some units.

No fossils.

Comments: this unit is a series of alternating sandstone beds. All beds are micaceous.

Sample(s): DM-10a,b,c,d,e,f,g.

\section{Unit 11}

$1.7 \mathrm{~m}$.

Calcareous Mudstone.

Red.

Sedimentary structures: pedogenic calcite veins throughout this unit (paleosol).

No fossils.

Sample(s): DM-11.

Unit 12

$0.60 \mathrm{~m}$.

Mudstone.

Olive tan.

No sedimentary structures.

No fossils.

Comments: primary difference between this unit and unit 11 is the color and lack of calcite veins.

Sample(s): DM-12.

\section{Unit 13}

$1.35 \mathrm{~m}$.

Limestone - silty / shaly argillaceous micrite.

Color: fresh - grey; weathers - tan.

Shaly beds $1-2 \mathrm{~cm}$.

Sedimentary structures: laminae visible throughout.

Fossils: few brachiopods, bivalves - Wilkingia, other - both vertical and horizontal trace fossils.

Sample(s): DM-13 *.

\section{Unit 14}

$1.86 \mathrm{~m}$.

Shale.

Olive at top and bottom, grey blue in middle.

Fissile.

No sedimentary structures.

Fossils: brachiopods and bivalves.

Sample(s): DM-14.

Unit 15 
$2.45 \mathrm{~m}$.

Limestone - argillaceous micrite.

Color: fresh - grey brown; weathers - brown tan.

Unit is composed of many smaller beds $(3 \mathrm{~cm}+)$.

No sedimentary structures.

Fossils: brachiopods - Anthracospirifer, Diaphragmus, Orthotetes, bivalves - Wilkingia, gastropods - Straparolus, pectins, other - crinoid columnals.

Sample(s): DM-15 *.

Unit 16

$1.1 \mathrm{~m}$.

Limestone - dirty argillaceous micrite grades up into a shaly limestone.

Color: fresh - grey brown; weathers - brown tan.

No sedimentary structures.

Fossils: brachiopods - Anthracospirifer, Compositia, Diaphragmus, Productus, bivalves Wilkingia.

Sample(s): DM-16*.

Unit 17

$1.65 \mathrm{~m}$.

Limestone - argillaceous micrite.

Color: fresh - grey brown; weathers - tan.

Unit is composed of many smaller beds.

No sedimentary structures.

Fossils: brachiopods - Anthracospirifer, Diaphragmus, Orthotetes, Productus, bivalves Wilkingia, pectins, other - trilobite tail, crinoid columnals.

Sample(s): DM-17*.

Unit 18

$3.05 \mathrm{~m}$.

Shaly Limestone.

Color: fresh - olive tan; weathers - tan brown.

Some argillaceous micrite interbeds.

No sedimentary structures.

Fossils: brachiopods - Anthracospirifer, Diaphragmus, Orthotetes, Productus, bivalves Wilkingia, pectins.

Sample(s): DM-18*.

Unit 19

$2.25 \mathrm{~m}$.

Limestone - argillaceous micrite.

Color: fresh - grey black; weathers - tan grey.

No sedimentary structures.

Fossils: brachiopods - Orthotetes, other - trilobite tail, crinoid columnals, fossil hash.

Sample(s): DM-19*.

Unit 20 
$1.65 \mathrm{~m}$.

Limestone Mystery Unit.

Can see this unit from road cut but cannot convincingly find it exposed anywhere else. It appears to be composed of many smaller beds $(5 \mathrm{~cm})$ with a gradational contact at base becoming more shaly at top. Everything above this unit is covered. 


\section{Droop Mountain}

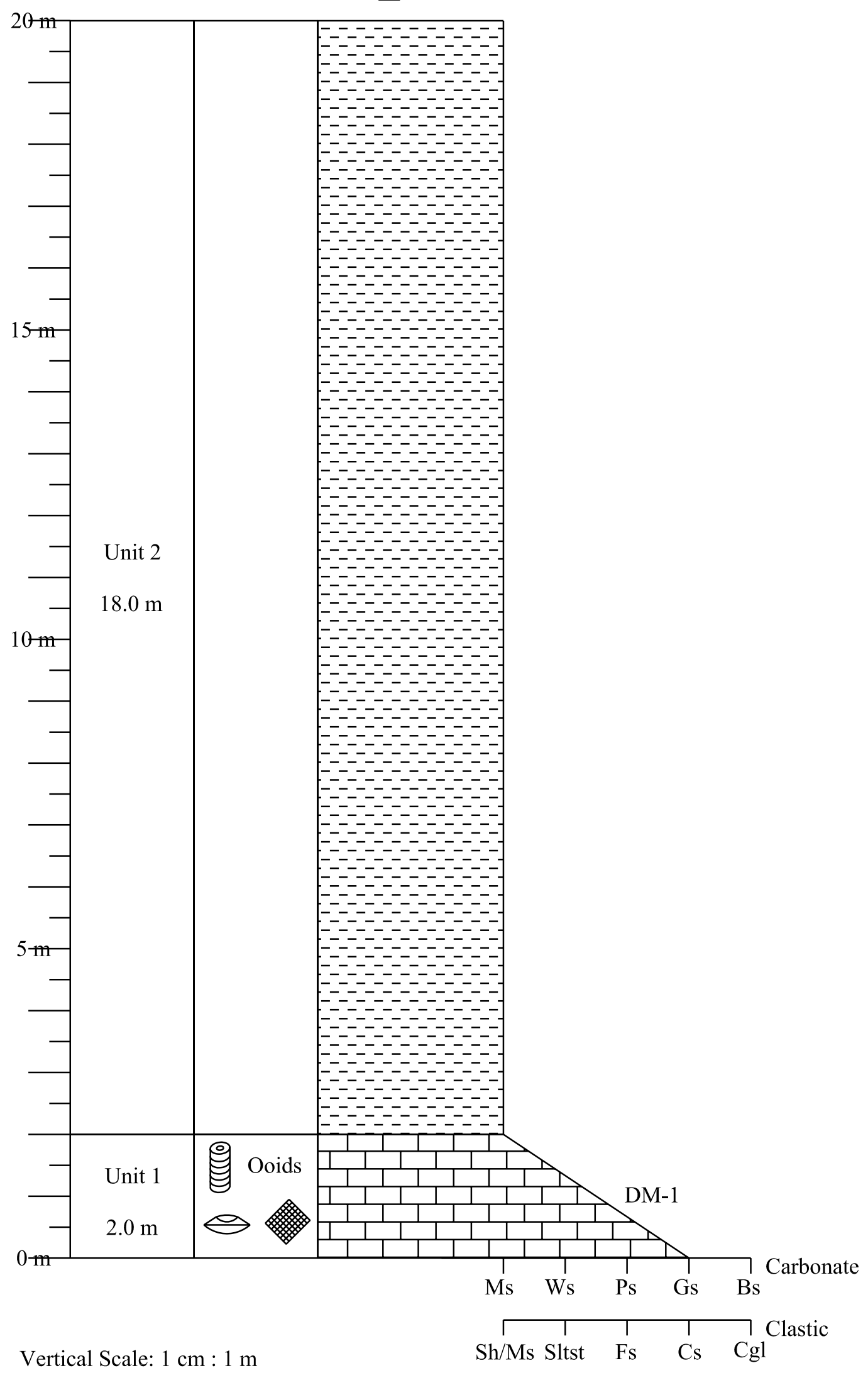




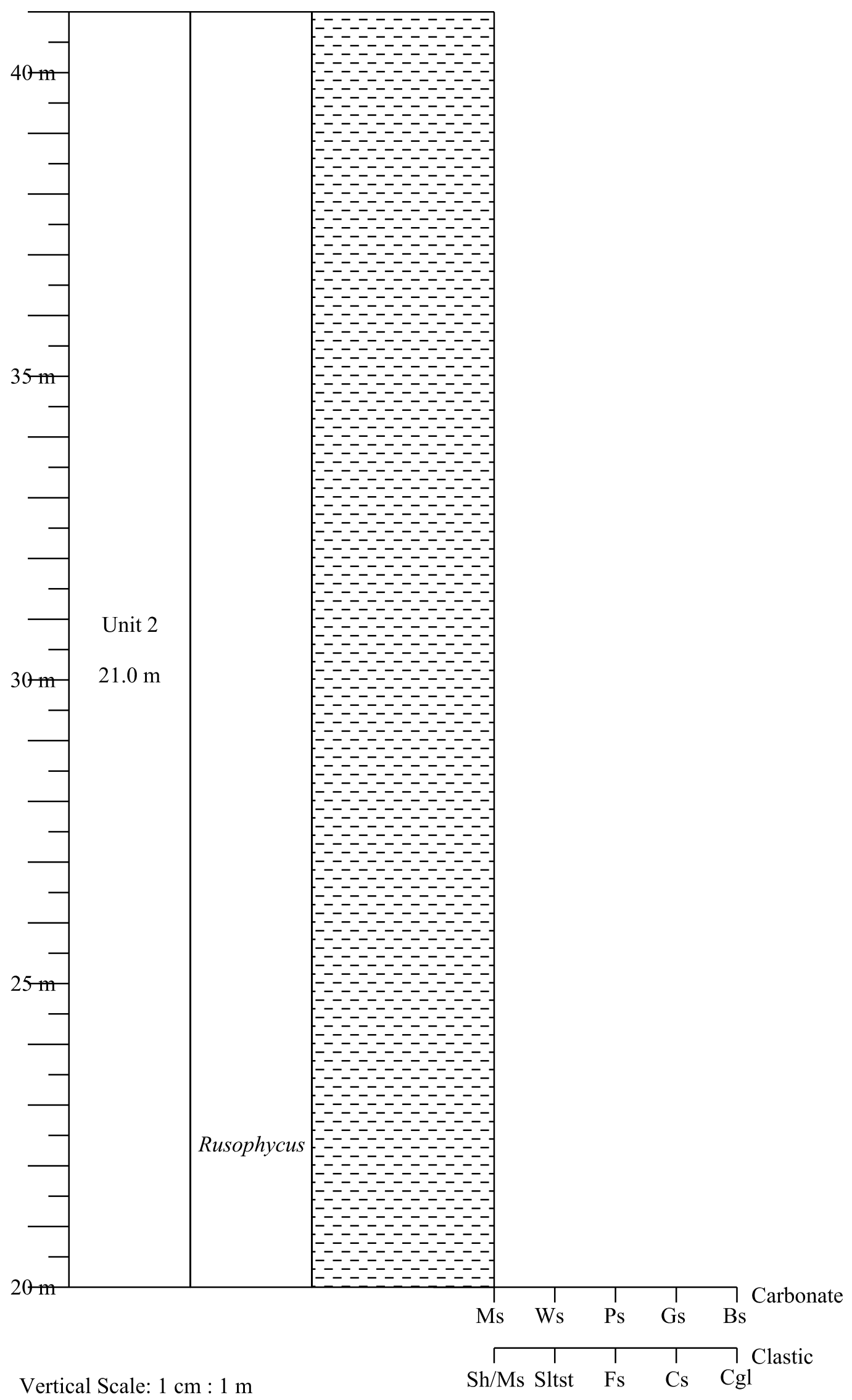




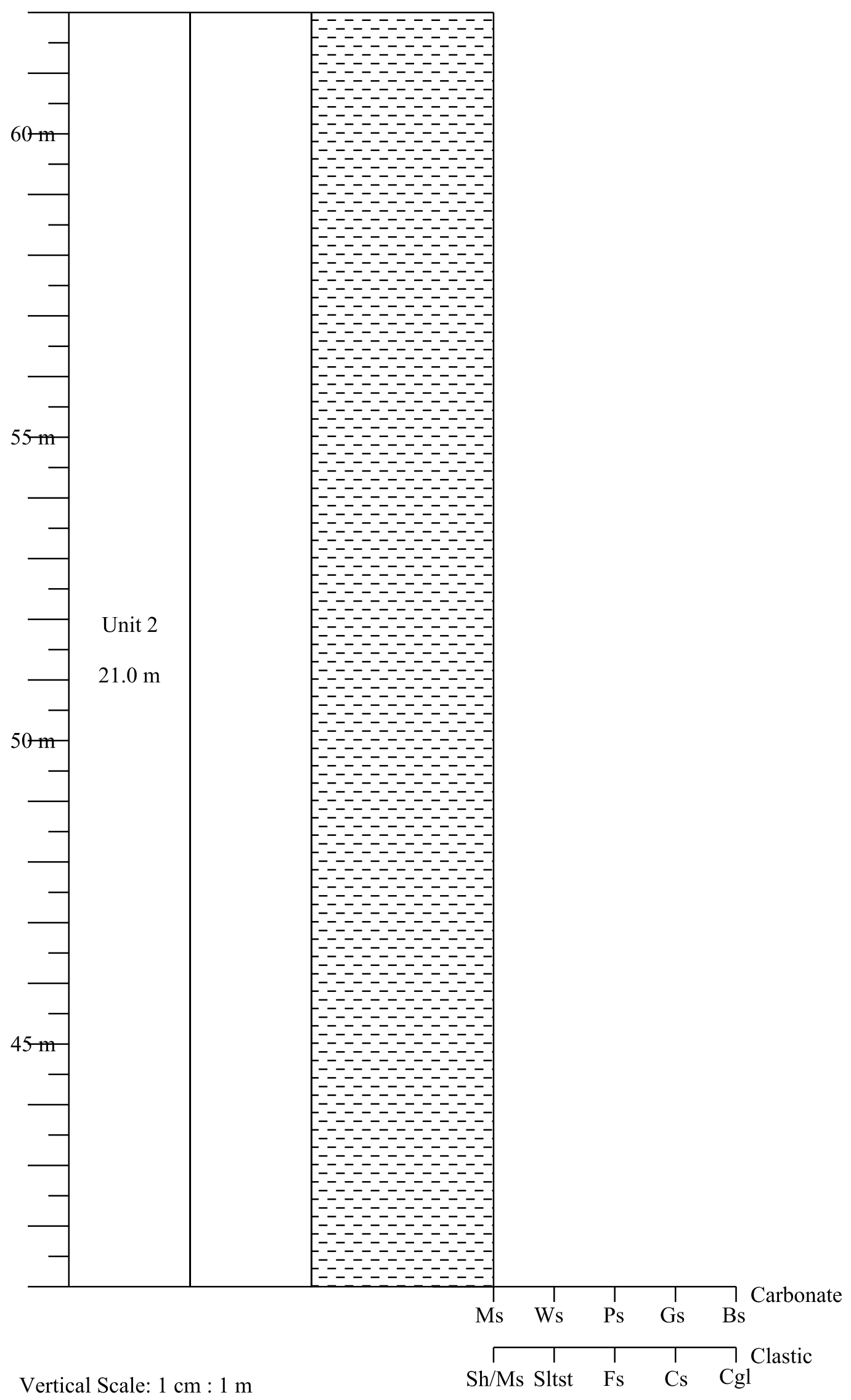




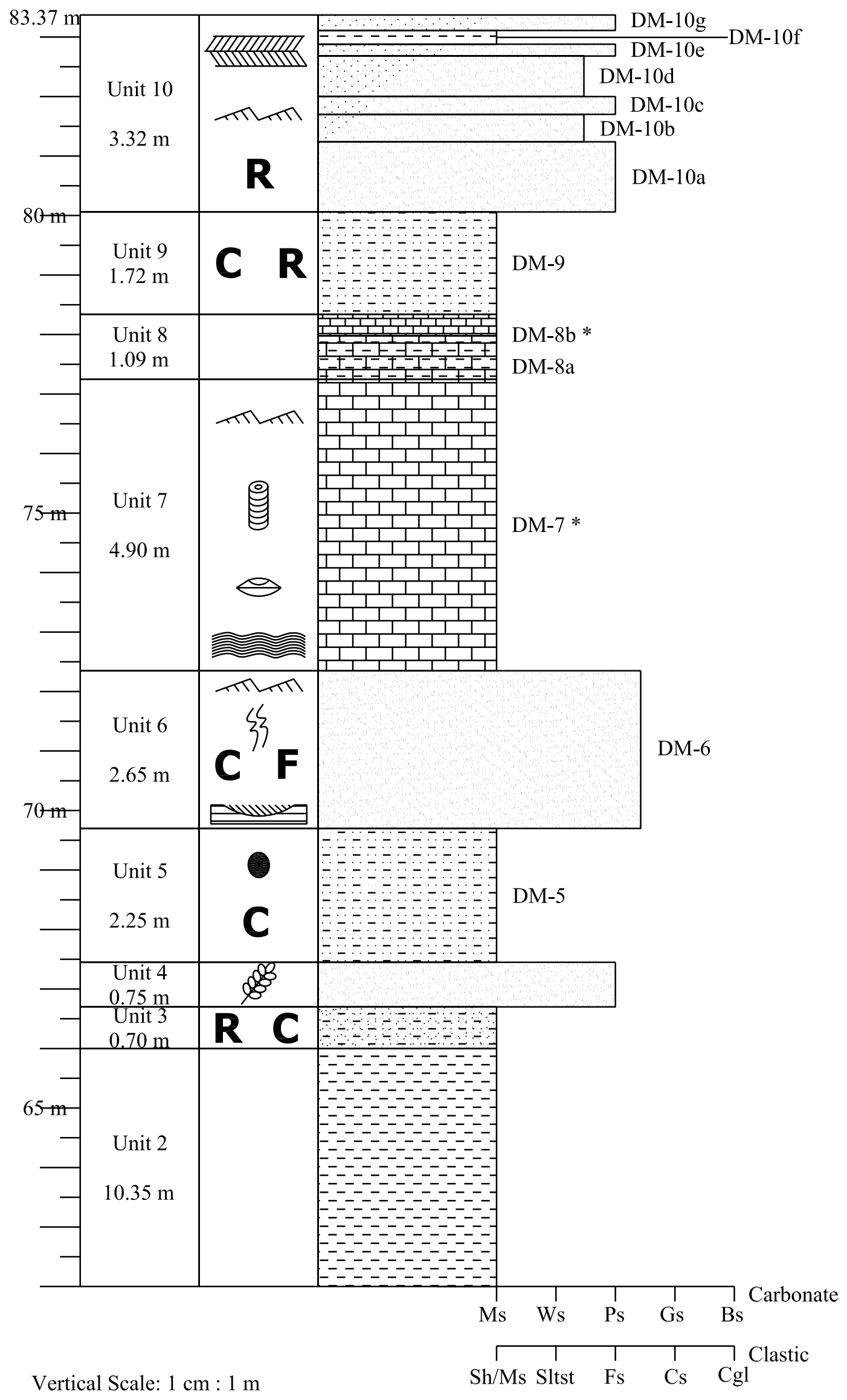




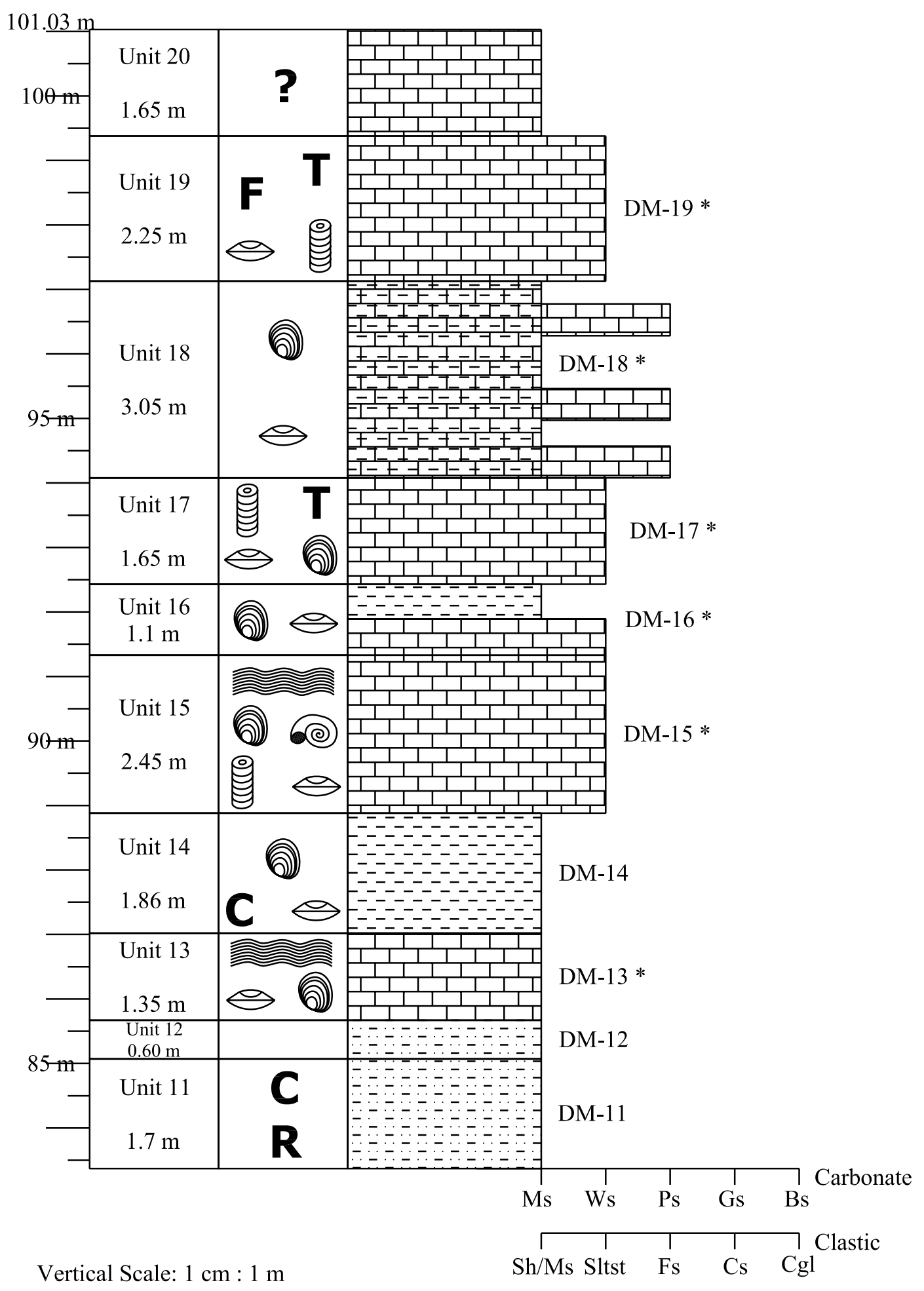




\section{APPENDIX 2}

Notes From Thin Sections During Point Counts 


\section{Locality: Hopwood}

Thin Section Number: HW-2

Date Identified: 09/21/04

Formation: Bluefield

Member: Reynolds

Rock Name: Wackestone
Locality: Hopwood

Thin Section Number: HW-5

Date Identified: 09/21/04

Formation: Bluefield

Member: Reynolds

Rock Name: Argillaceous Wackestone
Locality: Lake Lynn

Thin Section Number: LL-9

Date Identified: 09/21/04

Formation: Bluefield

Member: Reynolds

Rock Name: Skeletal Packstone

\begin{tabular}{|c|c|c|c|}
\hline 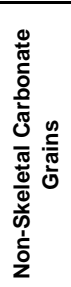 & |Peloids & $\begin{array}{l}\text { Oncolites } \\
\text { Intraclasts - carbonate aggregate grains cemented in } \\
\text { micrite with clay matrix. }\end{array}$ & \begin{tabular}{|l|} 
Peloids \\
Aggregate grains \\
Carbonate Intraclasts
\end{tabular} \\
\hline 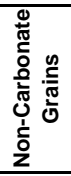 & \begin{tabular}{|l} 
Quartz \\
Clays \\
Micas \\
Phosphatic material \\
Pyrite - weathering rinds turn orange / brown with a \\
black center.
\end{tabular} & \begin{tabular}{|l|} 
Quartz \\
Micas \\
Clays \\
Chalcedony \\
Pyrite
\end{tabular} & \begin{tabular}{|l} 
Quartz \\
Micas \\
Clays \\
Phosphate \\
Pyrite
\end{tabular} \\
\hline 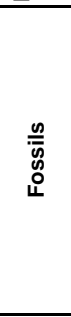 & $\begin{array}{l}\text { Bryozoans } \\
\text { Pelmatozoans } \\
\text { Ostracodes } \\
\text { Brachiopods } \\
\text { Brach. Spines } \\
\text { Punctate Brachs. } \\
\text { Echinoderm Spines } \\
\text { Gastropod } \\
\text { Bivalves } \\
\text { Algal Structures }\end{array}$ & \begin{tabular}{|l|} 
Bivalves \\
Ostracodes \\
Oncolites \\
Algal Structures \\
Fossil material recrystalized - few fossil grains - lots of \\
brown organic material - ?Bioerosion?
\end{tabular} & \begin{tabular}{|ll} 
Bivalves & Pelmatozoan Debris \\
Brachiopods & Forams \\
Pseudopunctate Brachs. & \\
Brach. Spines & \\
Punctate Brachs. & \\
Bryozoans & \\
Ostracodes & \\
Bored mollusc shell & \\
Algal Structures & \\
Echinoid Spines & \\
Trilobites & \\
\end{tabular} \\
\hline 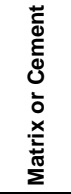 & $\begin{array}{l}\text { Lime mud matrix } \\
\text { Silt sized quartz grains }\end{array}$ & $\begin{array}{l}\text { Clay matrix with micas and quartz with carbonate clasts. } \\
\text { Unable to concretely identify many fossil grains in this } \\
\text { slide. }\end{array}$ & $\begin{array}{l}\text { Micrites - Neospar } \\
\text { Clays }\end{array}$ \\
\hline$\stackrel{\text { \& }}{2}$ & None & $\begin{array}{l}\text { Alternating laters of carbonate rich and clay rich } \\
\text { laminae. }\end{array}$ & Micro-stylolites. \\
\hline
\end{tabular}


Locality: Lake Lynn

Thin Section Number: LL-10

Rock Name: Skeletal Wackestone

\begin{tabular}{|c|c|c|c|}
\hline 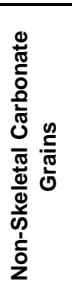 & $\begin{array}{l}\text { Peloids } \\
\text { Aggregate grains } \\
\text { Intraclasts } \\
\text { Calcispheres }\end{array}$ & \begin{tabular}{|l} 
Peloids \\
Aggregate grains \\
Intraclasts \\
Calcispheres
\end{tabular} & $\begin{array}{l}\text { Peloids (abundant) @ 4X } \\
\text { Calcispheres }\end{array}$ \\
\hline 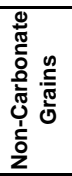 & \begin{tabular}{|l|} 
Quartz \\
Micas \\
Clays \\
Pyrite - in-fills appear to have replaced carb. Grains \\
Phosphate
\end{tabular} & \begin{tabular}{|l|} 
Quartz \\
Micas \\
Clays
\end{tabular} & \begin{tabular}{|l|} 
Quartz \\
Micas \\
Clays \\
Chert \\
Pyrite \\
Phosphate
\end{tabular} \\
\hline $\begin{array}{l}\stackrel{0}{\bar{n}} \\
\text { 足 } \\
\text { L }\end{array}$ & \begin{tabular}{|l|} 
Brachiopods \\
Punctate Brachs. \\
Pseudopunctate Brachs. \\
Brach. Spines \\
Ostracodes \\
Pelmatozoans \\
Bivalves \\
Echinoid Spines \\
Forams \\
Algal structures around grains and borings within grains. \\
Evidence of bioturbation in places. \\
\end{tabular} & \begin{tabular}{|ll} 
Brachiopods & Forams \\
Brach. Spines & Gastropods \\
Punctate Brachs. & \\
Bivalves & \\
Bryozoans & \\
Ostracodes & \\
Pelmatozoans & \\
Echinoid Spines & \\
Algal structures & \\
Trilobites & \\
Borings and micrite envelopes abundant. \\
\end{tabular} & $\begin{array}{l}\text { Brachiopods (few) } \\
\text { Punctate Brachs. } \\
\text { Bivalves } \\
\text { Ostracodes } \\
\text { Pelmatozoans } \\
\text { Trilobites } \\
\text { Forams } \\
\text { Algal Structures } \\
\text { Gastropods }\end{array}$ \\
\hline 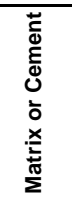 & $\begin{array}{l}\text { Lime mud matrix with lots of organics. } \\
\text { Sparry calcite cement in places. }\end{array}$ & Abundant micrite and clays & $\begin{array}{l}\text { Abundant micrite } \\
\text { Some microspar }\end{array}$ \\
\hline $\begin{array}{l}\text { \& } \\
\frac{0}{2}\end{array}$ & Evidence of micrite envelopes throughout. & None & None \\
\hline
\end{tabular}

Locality: Scherr

Thin Section Number: SH-6B - REJ

Date Identified: 10/05/04

Formation: Bluefield

Member: Reynolds

Member: Reynolds

Rock Name: Skeletal Wackestone

Date Identified: 10/05/04

Formation: Bluefield

.

Locality: Scherr

Tin Section Number: SH-6A

(

Date Identified: 09/23/04

Formation: Bluefield

Member: Reynolds

Intraclasts

Calcispheres

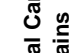

高

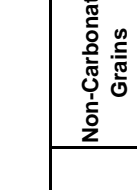

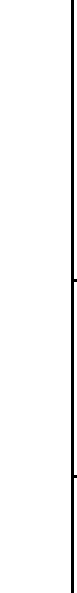

Algal structures around grains and borings within grains.

Evidence of bioturbation in places.

Lime mud matrix with lots of organics.

Rock Name: Peloidal Packstone 
Locality: Edray

Thin Section Number: ER-4C

Date Identified: 10/07/04

Formation: Bluefield

Member: Reynolds

Rock Name: Skeletal Wackestone
Locality: Edray

Thin Section Number: ER-5

Date Identified: 10/07/04

Formation: Bluefield

Member: Reynolds

Rock Name: Skeletal Wackestone
Locality: Edray

Thin Section Number: ER-6

Date Identified: 10/07/04

Formation: Bluefield

Member: Reynolds

Rock Name: Skeletal Wackestone / Packstone

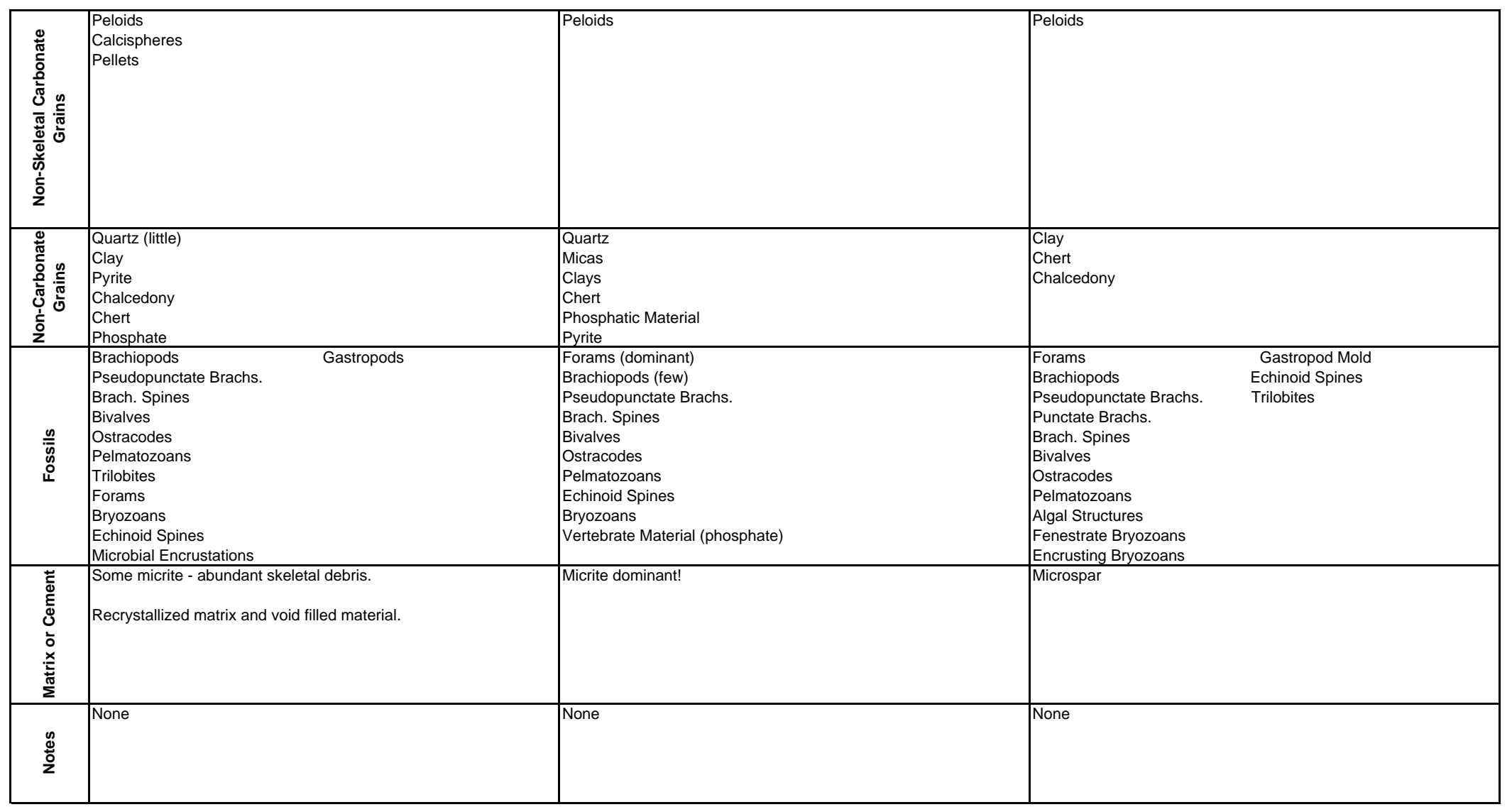


Locality: Edray

Thin Section Number: ER-7

Date Identified: 10/07/04

Formation: Bluefield

Member: Reynolds

Rock Name: Grainstone / Packstone
Locality: Edray

Thin Section Number: ER-9A - REJ

Date Identified: 10/07/04

Formation: Bluefield

Member: Reynolds

Rock Name: Skeletal Packstone
Locality: Edray

Thin Section Number: ER-9D

Date Identified: 10/07/04

Formation: Bluefield

Member: Reynolds

Rock Name: Skeletal Packstone

Peloids

Oncolites (viewed at 4X)

\begin{tabular}{|c|c|c|c|}
\hline 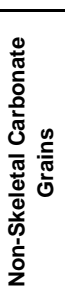 & Peloids & None & \begin{tabular}{|l} 
Peloids \\
Oncolites (viewed at $4 \mathrm{X}$ )
\end{tabular} \\
\hline 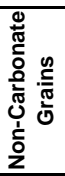 & $\begin{array}{l}\text { Phosphate } \\
\text { Pyrite } \\
\text { Chalcedony } \\
\text { Chert }\end{array}$ & $\begin{array}{l}\text { Quartz } \\
\text { Micas } \\
\text { Clays } \\
\text { Pyrite }\end{array}$ & \begin{tabular}{|l|} 
Quartz \\
Micas \\
Clays \\
Pyrite \\
Phosphate
\end{tabular} \\
\hline 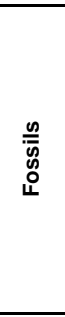 & \begin{tabular}{|ll} 
Globular Forams & Gastropod \\
Encrusting Forams & Echinoid Spines \\
Brachiopods & Trilobite \\
Pseudopunctate Brachs. & Encrusting Bryozoan \\
Brach. Spines & Fenestrate Bryozoans \\
Bivalves & \\
Algal Structures & Lots of Molds \\
Ostracodes & \\
Pelmatozoans & \\
Vertebrate Material (phosphate) \\
Conodont (clothespin shape) \\
\end{tabular} & \begin{tabular}{|l|} 
Brachiopods \\
Pseudopunctate Brachs. \\
Brach. Spines \\
Bivalves \\
Pelmatozoans \\
Bryozoans
\end{tabular} & \begin{tabular}{|ll} 
Bryozoans (lots) & Oncolites \\
Brachiopods & Trilobites \\
Brach. Spines & \\
Pseudopunctate Brachs. & \\
Bivalves & \\
Pelmatozoans & \\
Forams & \\
Ostracodes & \\
Echinoid Spines & \\
Girvanelid Algae & \\
Abundant bryozoan and algal debris. \\
\end{tabular} \\
\hline 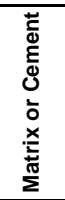 & Microspar & $\begin{array}{l}\text { Microspar } \\
\text { Some Shale }\end{array}$ & $\begin{array}{l}\text { Micrite sized quartz, clays, and micas. } \\
\text { Patchy microspar. }\end{array}$ \\
\hline$\stackrel{\mathscr{Q}}{\stackrel{0}{0}^{2}}$ & $\begin{array}{l}\text { Fracture across slide cuts through many fossils - filled } \\
\text { with neospar. } \\
\text { Micrite envelopes around grains. }\end{array}$ & Clay lined micro-stylolites. & $\begin{array}{l}\text { A slow sedimentation rate of clastics will still allow } \\
\text { normal marine fauna to exist in a lagoonal setting. }\end{array}$ \\
\hline
\end{tabular}


Locality: Edray

Thin Section Number: ER-9F - REJ

Date Identified: 10/12/04

Formation: Bluefield

Member: Reynolds

Rock Name: Skeletal-Wackestone
Locality: Droop Mountain

Thin Section Number: DM-7

Date Identified: 10/12/04

Formation: Bluefield

Member: Reynolds

Rock Name: Algal Boundstone
Locality: Droop Mountain

Thin Section Number: DM-8B

Date Identified: 10/12/04

Formation: Bluefield

Member: Reynolds

Rock Name: Calcareous Siltstone

\begin{tabular}{|c|c|c|c|}
\hline 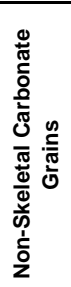 & $\begin{array}{l}\text { Calcispheres } \\
\text { Aggregate Grains }\end{array}$ & None & None \\
\hline 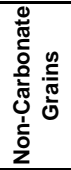 & $\begin{array}{l}\text { Quartz } \\
\text { Clays (abundant) } \\
\text { Pyrite } \\
\text { Phosphate }\end{array}$ & \begin{tabular}{|l|} 
Quartz \\
Clays \\
Micas \\
Phosphate \\
Chert
\end{tabular} & \begin{tabular}{|l|} 
Quartz \\
Clays \\
Micas \\
Pyrite \\
Dendritic staining pattern - iron oxide.
\end{tabular} \\
\hline 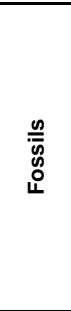 & \begin{tabular}{|l|} 
Brachiopods \\
Brach. Spines \\
Bivalves \\
Pelmatozoans \\
Forams \\
Ostracodes \\
Echinoid Spines \\
Trilobites \\
Trepostome Bryozoans \\
Gastropod \\
Some grains heavily bored.
\end{tabular} & $\begin{array}{l}\text { Algal Structures (dominant) } \\
\text { Foram } \\
\text { ?Sponge Spicules? }\end{array}$ & $\begin{array}{l}\text { No identifiable fossil remails. } \\
\text { Appear to be some plant fragments - questionable. }\end{array}$ \\
\hline 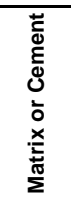 & Neospar & $\begin{array}{l}\text { Chert } \\
\text { Dolomite rhombs in chert (few). }\end{array}$ & Quartz and clay. \\
\hline $\begin{array}{l}\stackrel{y}{ \pm} \\
\frac{0}{2}\end{array}$ & $\begin{array}{l}\text { Slide ground too thin - too many holes - } \\
\text { DO NO USE THIS SLIDE IN POINT COUNT DATA. }\end{array}$ & $\begin{array}{l}\text { Micro-laminae present throughtout slide. } \\
\text { Slide has been called an algal boundstone because of } \\
\text { the amount of chert in the thin section. Once all chert is } \\
\text { removed from the data set the predominant structures }\end{array}$ & Micro-graded beds found throughout the slide. \\
\hline
\end{tabular}


Locality: Droop Mountain

Thin Section Number: DM-13 - REJ

Date Identified: 10/12/04

Formation: Bluefield

Member: Reynolds

Rock Name: Calcareous Siltstone
Locality: Droop Mountain

Thin Section Number: DM-15 - REJ

Date Identified: 10/13/04

Formation: Bluefield

Member: Reynolds

Rock Name: Argillaceous Skeletal-Wkst/Pkst
Locality: Droop Mountain

Thin Section Number: DM-16

Date Identified: 10/21/04

Formation: Bluefield

Member: Reynolds

Rock Name: Wackestone

\begin{tabular}{|c|c|c|c|}
\hline 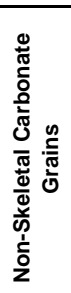 & Pellets (dominant) & Peloids & Calcispheres \\
\hline 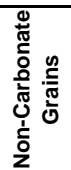 & \begin{tabular}{|l|} 
Quartz \\
Clays \\
Micas \\
Pyrite
\end{tabular} & $\begin{array}{l}\text { Quartz } \\
\text { Clays } \\
\text { Chert } \\
\text { Pyrite }\end{array}$ & $\begin{array}{l}\text { Quartz } \\
\text { Clays } \\
\text { Micas } \\
\text { Pyrite }\end{array}$ \\
\hline 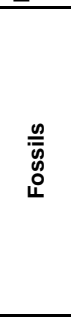 & \begin{tabular}{|l|} 
Pelmatozoans (few) \\
Bivalves \\
Ostracodes \\
Globular Forams \\
Brachiopods (few) \\
Trilobites
\end{tabular} & \begin{tabular}{|ll} 
Brachiopods & Oncolites \\
Brach. Spines & \\
Pseudopunctate Brachs. & \\
Bivalves & \\
Pelmatozoans & \\
Encrusting Bryozoans & \\
Ostracodes & \\
Forams & \\
Gastropod & \\
Echinoid Spines & \\
Algal Structures & \\
\end{tabular} & \begin{tabular}{|ll} 
Brachiopods & Echinoid Spines \\
Pseudopunctate Brachs. & \\
Brach. Spines & \\
Bivalves & \\
Pelmatozoans & \\
Bryozoans & \\
Ostracodes & \\
Forams & \\
Gastropod & \\
Trilobites & \\
Algal Structures & \\
\end{tabular} \\
\hline 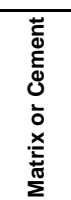 & $\begin{array}{l}\text { Quartz and clay dominant with some carbonate cement. } \\
\text { Some micrite and microspar. }\end{array}$ & $\begin{array}{l}\text { Clays and micrite. } \\
\text { Some microspar. }\end{array}$ & Micrite and microspar. \\
\hline $\begin{array}{l}\mathscr{d} \\
\text { оे }\end{array}$ & $\begin{array}{l}\text { Micro-graded beds found throughout the slide - visible at } \\
4 \mathrm{X} \text {. }\end{array}$ & None & None \\
\hline
\end{tabular}




\section{Thin Section Number: DM-17}

Date Identified: 10/21/04

Formation: Bluefield

Member: Reynolds

Rock Name: Skeletal-Wackestone/Packstone
Thin Section Number: DM-18

Date Identified: 10/22/04

Formation: Bluefield

Member: Reynolds

Rock Name: Peloidal Packstone
Thin Section Number: DM-19

Date Identified: $10 / 25 / 04$

Formation: Bluefield

Member: Reynolds

Rock Name: Skeletal-Wackestone

\begin{tabular}{|c|c|c|c|}
\hline 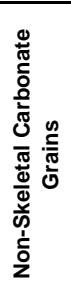 & Peloids & Peloids & Peloids \\
\hline 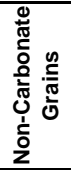 & \begin{tabular}{|l|} 
Quartz \\
Clays \\
Micas \\
Pyrite \\
Phosphate
\end{tabular} & \begin{tabular}{|l} 
Quartz \\
Clays \\
Pyrite
\end{tabular} & $\begin{array}{l}\text { Chalcecony } \\
\text { Pyrite } \\
\text { Barite } \\
\text { Gypsum }\end{array}$ \\
\hline $\begin{array}{l}\frac{0}{\bar{w}} \\
\bar{y} \\
0 \\
4\end{array}$ & \begin{tabular}{|ll} 
Brachiopods & Algal Encrusters \\
Punctate Brachs. & Echinoid Spines \\
Pseudopunctate Brachs. & \\
Brach. Spines & \\
Bivalves & \\
Pelmatozoans & \\
Bryozoans & \\
Ostracodes & \\
Forams & \\
Gastropod & \\
Trilobites & \\
\end{tabular} & \begin{tabular}{|l|} 
Brachiopods \\
Pseudopunctate \\
Brach. Spines \\
Pelmatozoans \\
Ostracodes \\
Forams \\
Echinoid Spines
\end{tabular} & \begin{tabular}{|l|} 
Brachiopods \\
Brach. Spines \\
Pseudopunctate Brachs. \\
Bivalves \\
Pelmatozoans \\
Forams \\
Echinoid Spines \\
Gastropods \\
Bryozoans - (some Fenestrates) \\
Ostacodes
\end{tabular} \\
\hline 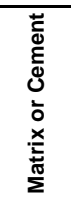 & Micrite and microspar. & $\begin{array}{l}\text { Sparry cement. } \\
\text { Some neospar (rare). }\end{array}$ & Micrite and microspar. \\
\hline ఖँ & None & $\begin{array}{l}\text { Laminae visible with naked eye and at low } \\
\text { magnification. }\end{array}$ & None \\
\hline
\end{tabular}




\section{APPENDIX 3}

Multivariate Quantitative Methods 


\section{MULTIVARIATE QUANTITATIVE METHODS}

\section{Description of Techniques}

Standard multivariate techniques were employed to aid in the determination of underlying patterns of similarity or dissimilarity within the data sets for the seven field localities and their various stratigraphic horizons. The multivariate technique used in this study is Q-mode multidimensional scaling analysis (MDS). This multivariate technique was obtained from the NTSYS-pc (Numerical Taxonomy System for the PC, Rholf, 1998) statistical software package.

MDS graphs produce plots that show the relationship between the selected variables or samples in two-dimensional Euclidean space. Distances between the points on the MDS plots are scaled so that the shortest distances equate to the greatest similarities (Kammer and Ausich, 1987). The stress of the graph measures the goodness-of-fit of the variables or samples to a two dimensional space. Values can range from zero to one with lower values indicating a very good fit (Rholf, 1998):

$\begin{array}{cl}0.0 & \text { Perfect } \\ 0.05 & \text { Excellent } \\ 0.10 & \text { Good } \\ 0.20 & \text { Fair } \\ >0.40 & \text { Poor }\end{array}$

Discussion and Analysis of Results

Data sets were arranged using all information gathered from thin-section identification and point-count analysis. Plots were generated considering all point-count data (Figure A3-1). As plots were generated and stress values were calculated it became necessary to collapse the data sets by combining variables into groupings. In order to decrease the stress values, all 


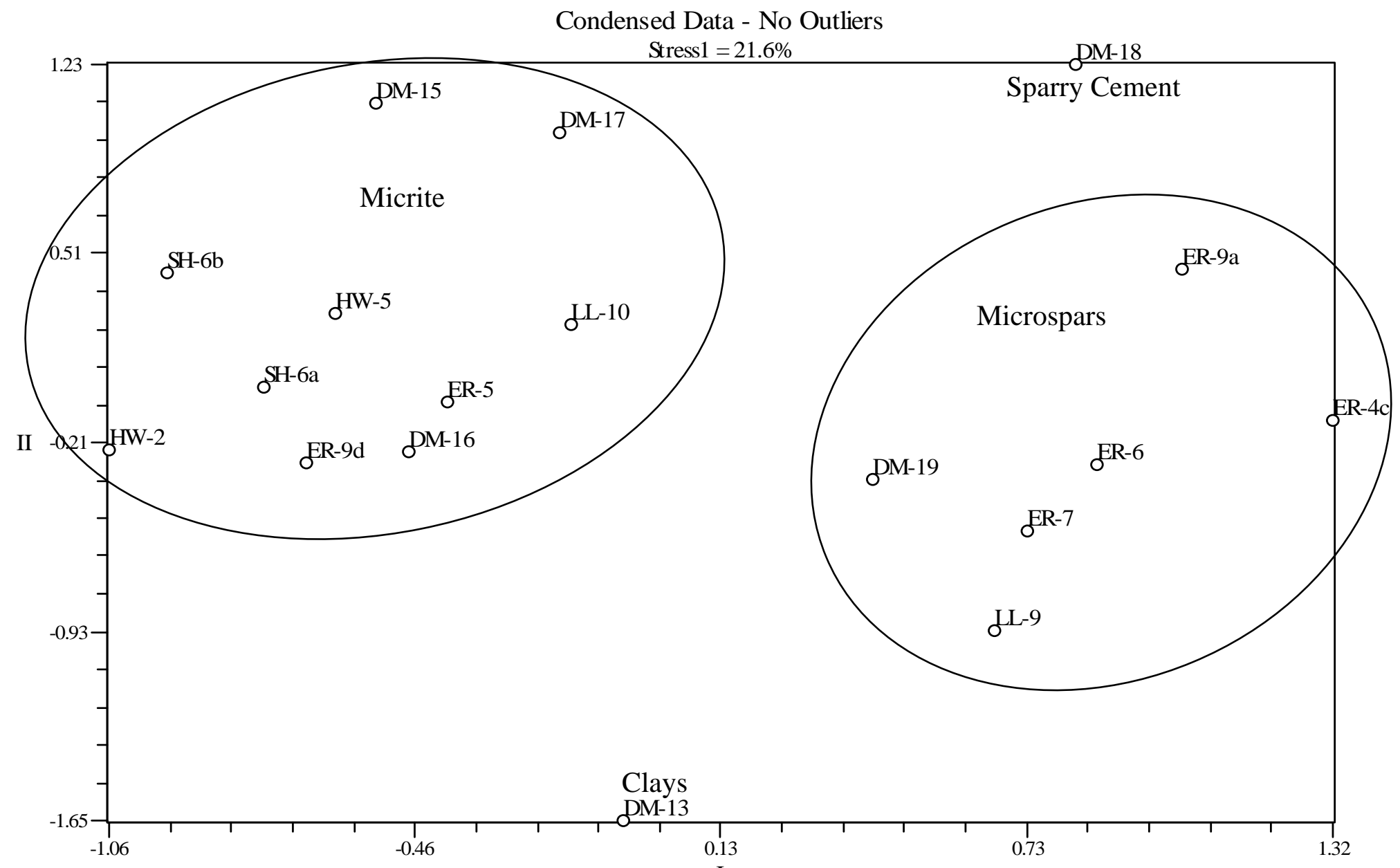

Figure A3-1. Q-mode analysis of outcrop samples based on fossil, matrix, and cement data. The data set for this MDS plot has been condensed so that only syndepositional factors were considered in the analysis (Appendix 4a). Units that plotted on the outlying fringes have been removed to help decrease the stress value. The groups in this plot are arranged by the dominant matrix type. 
diagenetic and post-depositional variables were removed (Figure A3-2). Only syndepositional variables are considered in these analyses.

Binary data analyses of fossil identifications at the generic level were attempted from outcrop descriptions. Stress values for this data were well beyond the "poor” category of Rholf's goodness-of-fit. Therefore it has been determined that binary data are insufficient to recognize patterns of faunal change within this data set.

Q-mode analysis of figure A3-1 illustrates how the units respond to MDS analysis of the condensed data set - including fossil, matrix, and cement percentage data. The stress value for this data set is $21.6 \%$ - fair to poor (Rholf, 1998). The overwhelming control on the arrangement and grouping of this data is the matrix material and cement types. The four point clusters have been divided from that into: clays, microspar, micrite, and sparry cement. A definite gradation can be seen in the point clusters. From top to bottom, the increase of clastic material can be seen. This pattern most likely results from syndepositional environmental factors controlling the grain types being deposited. The ranges between these points and point clusters may explain the elevated goodness-of-fit number.

Q-mode analysis of figure A3-2 shows two trends within the MDS point clusters. The first shows that the amount of clastic influence in the units is decreasing from left to right. The second shows marine conditions improving from bottom to top. Units occurring in the middle of the right side all have faunal diversities representing normal marine conditions. The trends in this MDS plot reflect those of the outcrops. The more normal marine units are found in the most southern outcrops where deeper water conditions allowed normal marine conditions to persist for longer periods of time. 
Thin Section Data - Fossils, No Cement, No Outliers

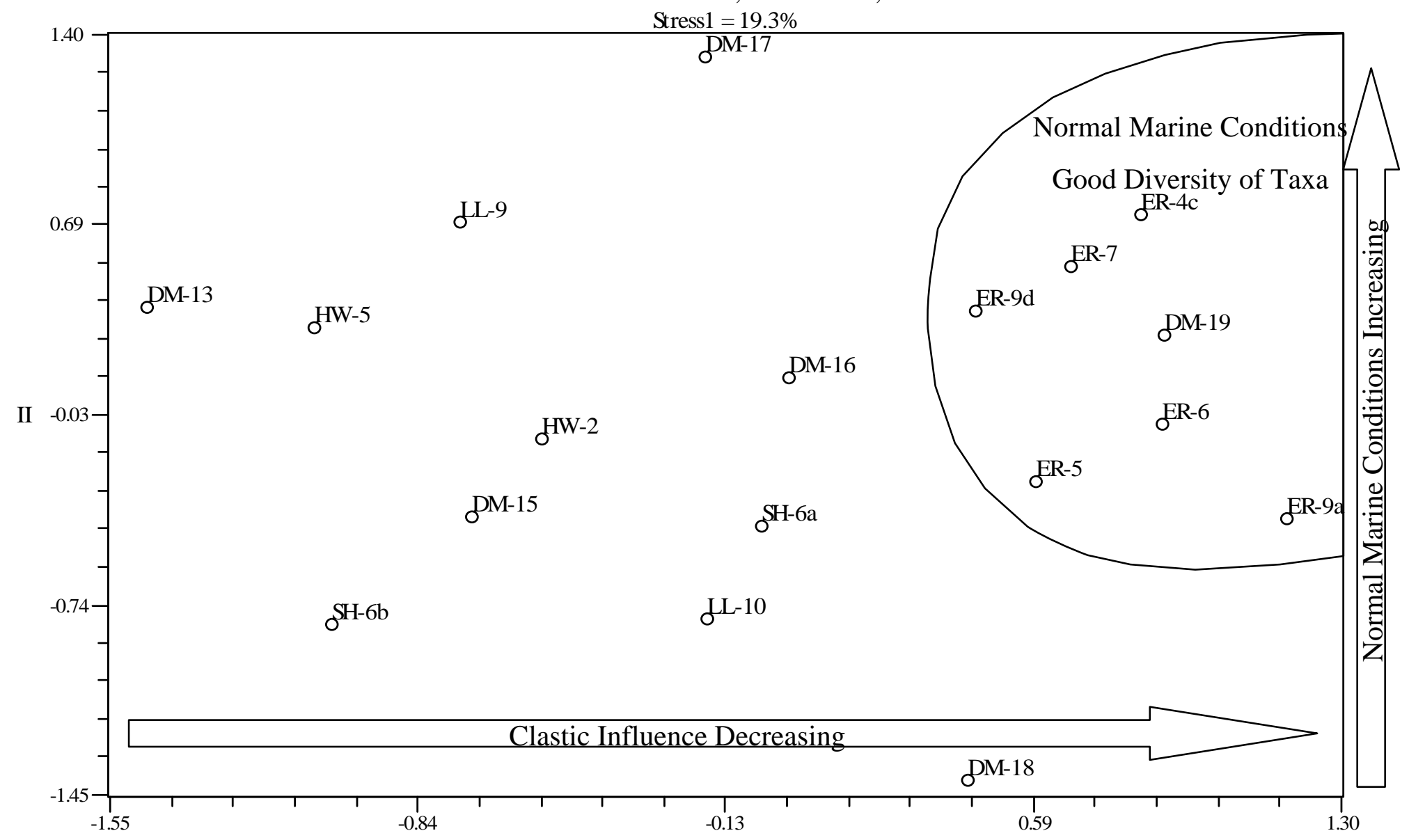

Figure A3-2. Q-mode analysis of outcrop trends based on the normalized percentages of fossil data. The data set for this MDS plot has been condensed so that only fossil data were considered in the analysis (Appendix 4b). Units that plotted on the outlying fringes have been removed to help decrease the stress value. The groups in this plot are arranged based on the fossil data and reflect environmental conditions decreasing in clastic influence and increasing normal marine conditions 
In order to decrease the stress value, the data set was collapsed so that only fossil data and syndepositional environmental conditions were considered. The matrix and cement data have been removed in order to achieve a better stress value. Removing the overwhelming numbers of the matrix and cement data allowed the taxonomic data to emerge from the noise of the other variables. Using only the taxonomic data allowed a new graph to be constructed considering different characteristics while using the same basic data set. This data was then normalized - once all the undesired groups were removed (data collapsed) the totals for each sample were re-calculated and then percentages of the new total used in the creation of this graph. The stress value for this data set is $19.3 \%$ - good to fair (Rholf, 1998). 
APPENDIX 4

MDS Data 


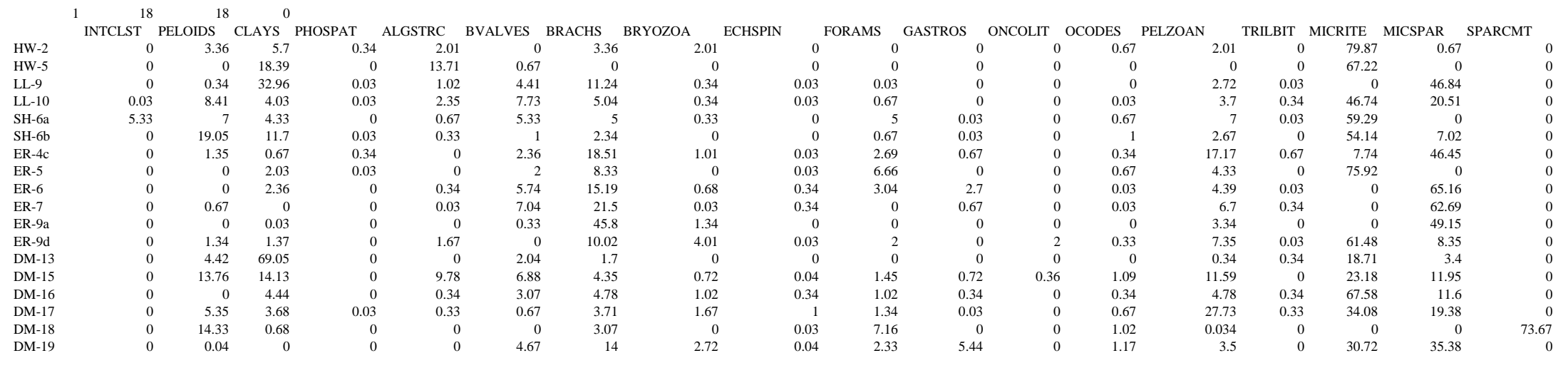




\begin{tabular}{|c|c|c|c|c|c|c|c|c|c|c|c|c|c|c|c|}
\hline & INTCLST $^{18}$ & PELOIDS 15 & PHOSPHAT ${ }^{0}$ CLASTIC & & ALGSTRC & BIVALVES & BRACHS & BRYOZOA & ECHSPIN & FORAMS & GASTROS & ONCOLIT & OCODES & PELZOAN & TRILBIT \\
\hline $\mathrm{HW}-2$ & 0.00 & 3.33 & 0.33 & 5.67 & 2.00 & 0.00 & 3.33 & $\begin{array}{l}\mathrm{BRYOLOA} \\
2.00\end{array}$ & ECHSPIN 0.00 & $\begin{array}{l}\text { FURAMS } \\
0.00\end{array}$ & $\begin{array}{l}\text { GASIRUS } \\
0.00\end{array}$ & 0.00 & 0.67 & $\begin{array}{r}\text { PELLOAN } \\
2.00\end{array}$ & IRILB11 0.00 \\
\hline HW-5 & 0.00 & 0.00 & 0.00 & 18.33 & 13.67 & 0.67 & 0.00 & 0.00 & 0.00 & 0.00 & 0.00 & 0.00 & 0.00 & 0.00 & 0.00 \\
\hline LL-9 & 0.00 & 0.33 & 0.03 & 32.37 & 1.00 & 4.33 & 11.03 & 0.33 & 0.03 & 0.03 & 0.00 & 0.00 & 0.00 & 2.67 & 0.03 \\
\hline LL-10 & 0.03 & 8.33 & 0.03 & 4.00 & 2.33 & 7.67 & 5.00 & 0.33 & 0.03 & 0.67 & 0.00 & 0.00 & 0.03 & 3.67 & 0.33 \\
\hline SH-6a & 5.33 & 7.00 & 0.00 & 4.33 & 0.67 & 5.33 & 5.00 & 0.33 & 0.00 & 5.00 & 0.03 & 0.00 & 0.67 & 7.00 & 0.03 \\
\hline SH-6b & 0.00 & 19.00 & 0.03 & 11.67 & 0.33 & 1.00 & 2.33 & 0.00 & 0.00 & 0.67 & 0.03 & 0.00 & 1.00 & 2.67 & 0.00 \\
\hline ER-4c & 0.00 & 1.33 & 0.33 & 0.67 & 0.00 & 2.33 & 18.33 & 1.00 & 0.03 & 2.67 & 0.67 & 0.00 & 0.33 & 17.00 & 0.67 \\
\hline ER-5 & 0.00 & 0.00 & 0.03 & 2.03 & 0.00 & 2.00 & 8.33 & 0.00 & 0.03 & 6.67 & 0.00 & 0.00 & 0.67 & 4.33 & 0.00 \\
\hline ER-6 & 0.00 & 0.00 & 0.00 & 2.33 & 0.33 & 5.67 & 15.00 & 0.67 & 0.33 & 3.00 & 2.67 & 0.00 & 0.03 & 4.33 & 0.03 \\
\hline ER-7 & 0.00 & 0.67 & 0.00 & 0.00 & 0.03 & 7.00 & 21.33 & 0.03 & 0.33 & 0.00 & 0.67 & 0.00 & 0.03 & 6.67 & 0.33 \\
\hline ER-9a & 0.00 & 0.00 & 0.00 & 0.03 & 0.00 & 0.33 & 45.67 & 1.33 & 0.00 & 0.00 & 0.00 & 0.00 & 0.00 & 3.33 & 0.00 \\
\hline ER-9d & 0.00 & 1.33 & 0.00 & 1.37 & 1.67 & 0.00 & 10.00 & 4.00 & 0.03 & 2.00 & 0.00 & 2.00 & 0.33 & 7.33 & 0.03 \\
\hline DM-13 & 0.00 & 4.33 & 0.00 & 67.67 & 0.00 & 2.00 & 1.67 & 0.00 & 0.00 & 0.00 & 0.00 & 0.00 & 0.00 & 0.33 & 0.33 \\
\hline DM-17 & 0.00 & $\begin{array}{r}5.33 \\
\end{array}$ & 0.03 & $\begin{array}{l}3.67 \\
0.67\end{array}$ & 0.33 & 0.67 & 3.70 & 1.67 & 1.00 & 1.33 & 0.03 & 0.00 & 0.67 & 27.67 & \\
\hline $\begin{array}{l}\text { DM-18 } \\
\text { DM-19 }\end{array}$ & $\begin{array}{l}0.00 \\
0.00\end{array}$ & $\begin{array}{r}14.00 \\
0.03\end{array}$ & $\begin{array}{l}0.00 \\
0.00\end{array}$ & $\begin{array}{l}0.67 \\
0.00\end{array}$ & $\begin{array}{l}0.00 \\
0.00\end{array}$ & $\begin{array}{l}0.00 \\
400\end{array}$ & $\begin{array}{r}3.00 \\
1200\end{array}$ & $\begin{array}{l}0.00 \\
2.33\end{array}$ & $\begin{array}{l}0.03 \\
0.03\end{array}$ & $\begin{array}{l}7.00 \\
200\end{array}$ & $\begin{array}{l}0.00 \\
467\end{array}$ & $\begin{array}{l}0.00 \\
0.00\end{array}$ & $\begin{array}{l}1.00 \\
1.00\end{array}$ & $\begin{array}{l}0.03 \\
3.00\end{array}$ & $\begin{array}{l}0.00 \\
0.00\end{array}$ \\
\hline
\end{tabular}




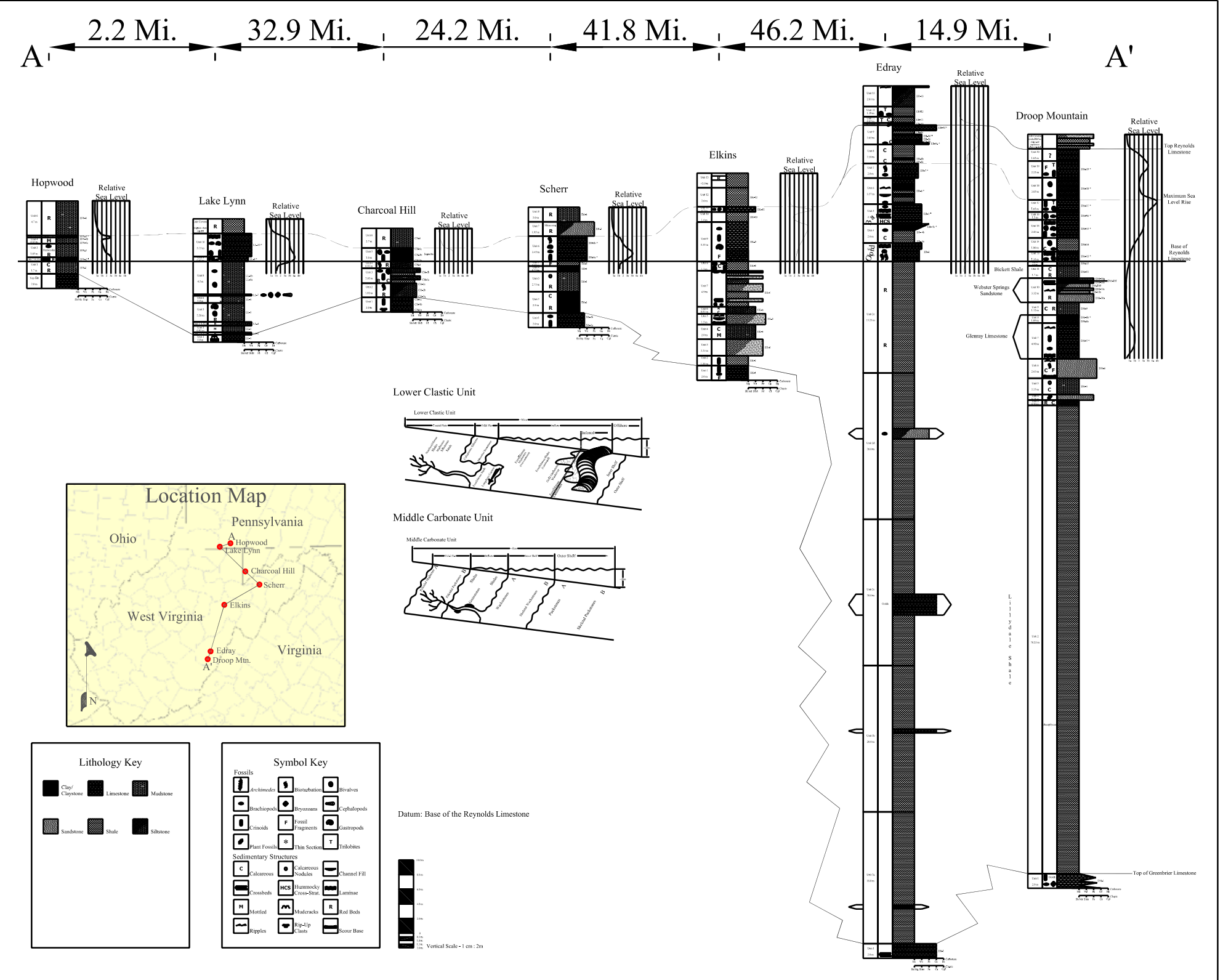

